\title{
The Development of Bipolar Log-Domain Filters in a Standard CMOS Process
}

\author{
Geoffrey Duerden
}

(B.A.Sc. 1998)

Department of Electrical Engineering

McGill University, Montréal

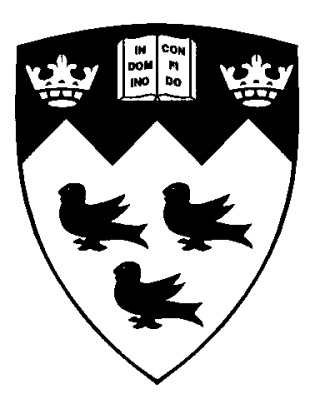

December 2001

A thesis submitted to the Faculty of Graduate Studies and Research in partial fulfillment of the requirements for the degree of Master of Engineering 
National Library of Canada

Acquisitions and Bibliographic Senvices

305 Wellington Strept Oriawe ON KIA ONM Cansod
Bibliotheque nationale du Canada

Acquisitions el services bibliographiques

305, no Wellington Oriam ON KIA ONA Caned

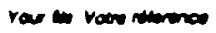

The author has granted a nonexclusive licence allowing the National Library of Canada to reproduce, loan, distribute or sell copies of this thesis in microform, paper or electronic formats.

The author retains ownership of the copyright in this thesis. Neither the thesis nor substantial extracts from it may be printed or otherwise reproduced without the author's permission.
L'auteur a accordé une licence non exclusive permettant à la Bibliotheque nationale du Canada de reproduire, prêter, distribuer ou vendre des copies de cette thèse sous la forme de microfiche/film, de reproduction sur papier ou sur format électronique.

L'auteur conserve la propriété du droit d'auteur qui protège cette thèse. $\mathrm{Ni}$ la thèse ni des extraits substantiels de celle-ci ne doivent être imprimés ou autrement reproduits sans son autorisation. 


\section{Abstract}

Log-domain filters have emerged in recent years as a new and important class of continuous-time filter. The attractive features of these filters include their compact structure, their potential to run at high frequencies while operating from low power supplies, and their electronic tunability. At the heart of the log-domain filtering technique is the logarithmic / exponential relationship between voltage and current in a transistor. In this work, the development of log-domain filters in CMOS technology will be investigated. The lateral bipolar transistor, inherent to CMOS processes, will be used for this purpose.

A SPICE compatible model for a lateral PNP transistor, fabricated in $0.35 \mu \mathrm{CMOS}$ technology, is presented. A log-domain integrator, which makes use of both lateral PNP transistors and MOSFET transistors operating in strong inversion, as well as a biquadratic low-pass log-domain filter and a third order elliptic low-pass log-domain filter, have each been designed and characterized in $0.35 \mu \mathrm{CMOS}$ technology. Experimental results indicate that these filters are capable of operation at frequencies up to $10 \mathrm{MHz}$. For the elliptic filter operating at a bias current of $10 \mu \mathrm{A}$, the experimentally measured total harmonic distortion is $-39.6 \mathrm{~dB}$ for an input current of $5 \mu \mathrm{A}$ (a $50 \%$ current modulation index), the dynamic range is $-34.1 \mathrm{~dB}$, and the total power consumption is $183 \mu \mathrm{W} /$ pole from a $2.5 \mathrm{~V}$ supply. These filters are capable of operating at significantly higher frequencies than CMOS logdomain filters described in the literature which make use of MOSFET transistors operating in weak inversion. 


\section{Résumé}

Ces dernières années ont vu les filtres logarithmiques s'imposer comme classe importante des filtres continus. L'attrait pour cette classe de filtres réside, entre autres, dans leur structure compacte, leur potentiel a opérer en très haute fréquence sous faible puissance d'alimentation ainsi que leur "réglabilite". Au coeur de la technique de filtrage logarithmique se trouve la relation logarithmique / exponentielle entre potentiel et courant dans un transistor. Dans ce travail, nous étudions le développement des filtres logarithmique en technologie CMOS. Le transistor bipolaire latéral inhérent aux processus CMOS est utilisé a cette fin.

Un modèle du transistor latéral PNP fabriqué dans la technologie CMOS $0.35 \mu \mathrm{m}$ et compatible avec SPICE est présenté. Un intégrateur logarithmique, utilisant à la fois des transistors bipolaires PNP latéraux ainsi que des MOSFETs en régime d'inversion forte, un filtre logarithmique biquadratique passe bas et un filtre logarithmique elliptique passe bas du troisième ordre on été conçus et characterisés en technologie CMOS $0.35 \mu \mathrm{m}$. Les résultats expérimentaux indiquent que ces filtres sont capables d'opérer à des fréquences allant jusqu'a $10 \mathrm{MHz}$. Pour le filtre elliptique à $10 \mu \mathrm{A}$ de courant de polarisation, la distorsion harmonique totale (THD) mesurée expérimentalement est de $-39.6 \mathrm{~dB}$ pour un courant d'entrée de $5 \mu \mathrm{A}$ (indicede modulation de courant de 50\%), l'étendu dynamique est de $-34.1 \mathrm{~dB}$ et la consommation totale de puissance est de $183 \mu \mathrm{W}$ pour une alimentation de $2.5 \mathrm{~V}$. Ces filtres sont capables d'opérer à des fréquences significativement plus hautes que les filtres logarithmiques CMOS décrit dans la littérature ou les transitors MOSFET opèrent en régime d'inversion faible. 


\section{Dedication}

To Margaret, for your care and wisdom which has pulled me through. 


\section{Acknowledgements}

First and foremost, I would like to express my gratitude to my supervisor Professor Gordon Roberts, not only for his guidance, energy, and enthusiasm, but also for his patience, and for the freedoms he has granted me throughout my years of study.

I also wish to thank all members of the MACS lab, and in particular Mohamed Hafed and Sebastien Laberge for helping me pull through some particularly late nights and tight deadlines, and Arshan Aga, Mona Safi-Harb, Mourad Oulmane, Naveen Chandra, and Nazmy Abaskharoun for their help, advice, and encouragement. I also wish to thank Prof. Mourad El-Gamal for his many insights into log-domain filters and circuits in general.

I would also like to thank Ricky Der, for countless fascinating discussions which constantly remind me of why I enjoy being at university, and Lana Fisher, for her unwavering friendship, particularly in times of need.

Last but not least, I would like to acknowledge my family for their solid support of all my endeavours. 


\section{Table of Contents}

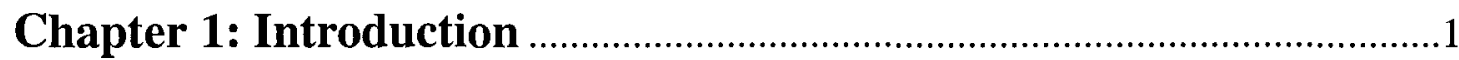

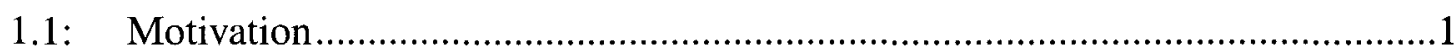

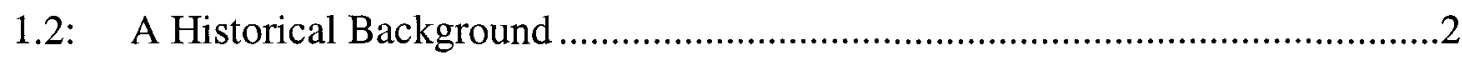

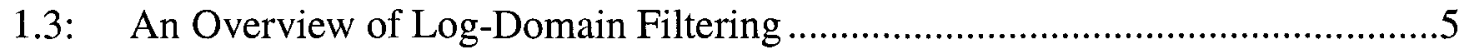

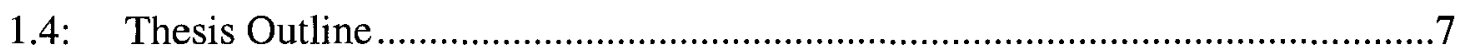

Chapter 2: Modelling Lateral PNP Transistors in

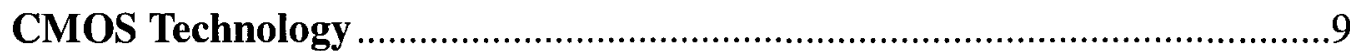

2.1: Lateral PNP Transistors: Structure and Layout ..........................................10

2.2: Lateral PNP Transistors: SPICE Model Parameters........................................13

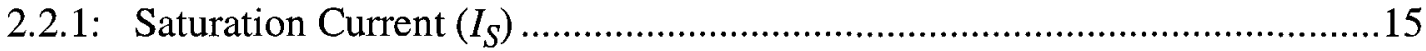

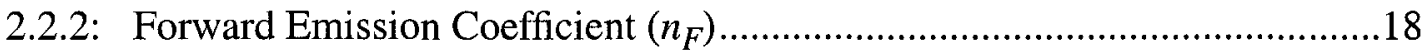

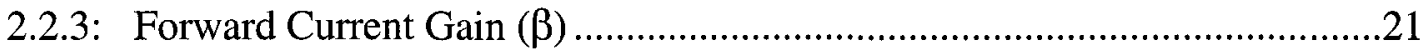

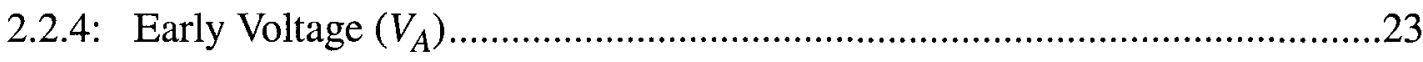

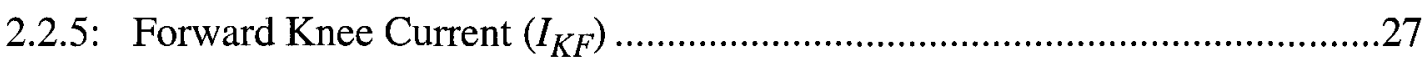

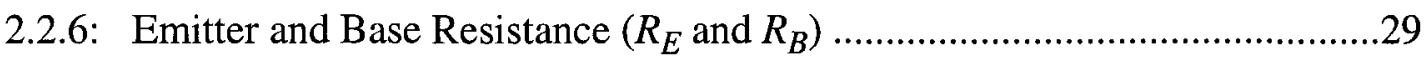

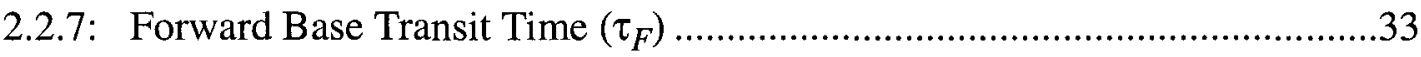

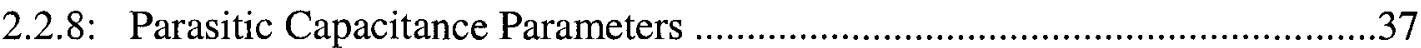

2.3: Lateral PNP Transistors: Complete SPICE Model ........................................38

\section{Chapter 3: The Design of a Log-Domain Integrator in}

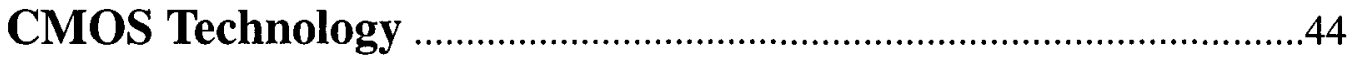

3.1: Fundamentals of Log-Domain Integrator Design ......................................45

3.1.1: The Log-Domain Cell and the LOG(x) and EXP(x) Operators..................45

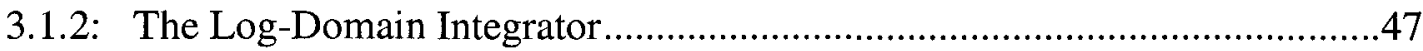

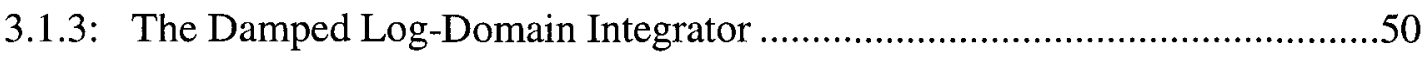

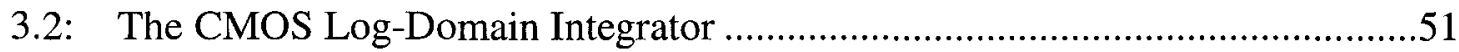

3.2.1: Mapping the Log-Domain Integrator in CMOS Technology ......................51

3.2.2: Input Compression / Output Expansion and Interface Circuitry ..................55

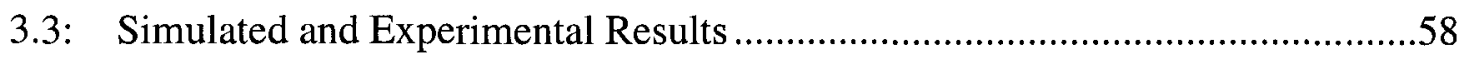

3.3.1: Description of the Log-Domain Integrator Test Circuit ............................59 


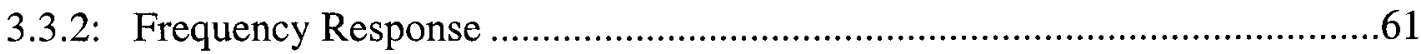

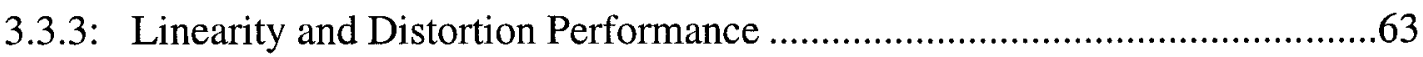

3.3.4: Noise Performance...................................................................................65

3.3.5: Summary of the Damped Integrator Performance ........................................68

\section{Chapter 4: The Design of Bipolar Log-Domain Filters in}

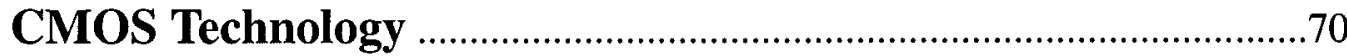

4.1: Fundamentals of Log-Domain Filter Design ..................................................71

4.1.1: An Overview of the Operational Simulation of LC Ladders ........................71

4.1.2: Operational Simulation for Log-Domain Filters ..........................................74

4.2: The Design of Second and Third Order Log-Domain Filters ..............................76

4.2.1: A Low Pass Biquadratic Log-Domain Filter ..............................................76

4.2.2: A Low Pass Third Order Elliptic Log-Domain Filter ....................................79

4.3: Experimental Results ....................................................................................8

4.3.1: Description of the Log-Domain Filter Test Circuits ......................................84

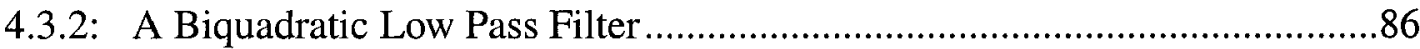

4.3.3: A Third Order Elliptic Low Pass Filter.......................................................91

4.4: A Comparison of Filter Performance ………….............................................95

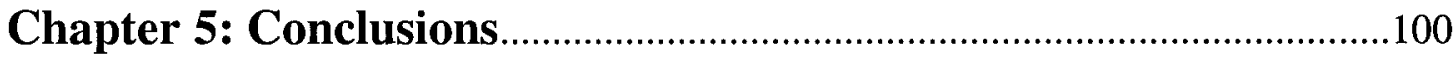

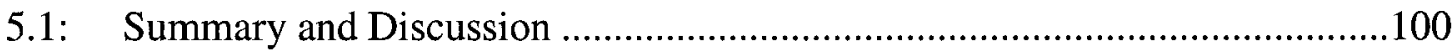

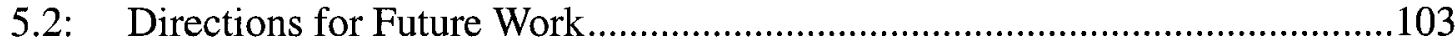

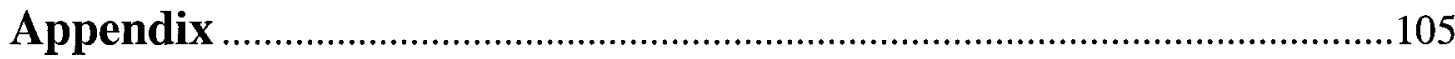

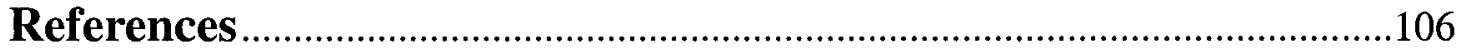




\section{List of Figures}

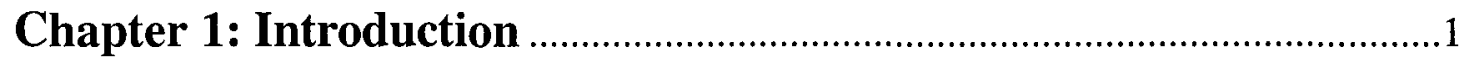

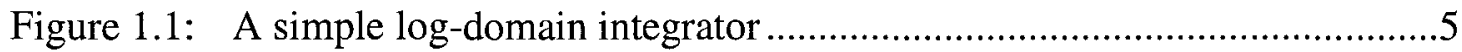

Figure 1.2: Block diagram of a log-domain filter .................................................

\section{Chapter 2: Modelling Lateral PNP Transistors in}

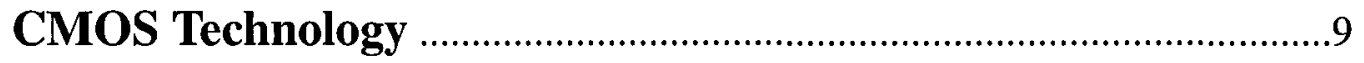

Figure 2.1: Cross section of a vertical and lateral PNP transistor ............................10

Figure 2.2: Schematic and symbolic representation of a lateral PNP device ...........11

Figure 2.3: Layout of a minimum size $0.35 \mu$ CMOS lateral PNP transistor .............12

Figure 2.4: Comparison of experimentally measured collector currents ...................17

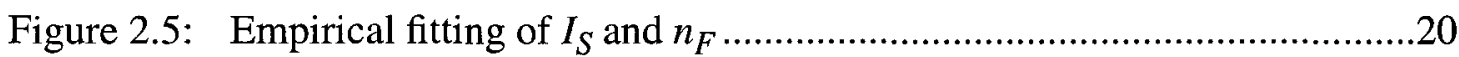

Figure 2.6: Comparison of modelled and measured base and collector currents......23

Figure 2.7: Measured base and collector currents with varying $V_{C B} \ldots \ldots \ldots \ldots \ldots \ldots \ldots \ldots . .25$

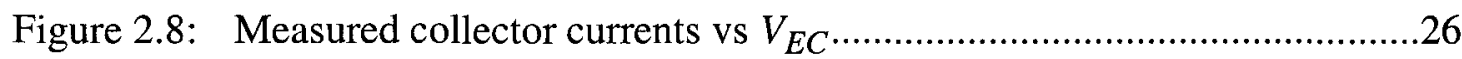

Figure 2.9: Representation of ideal collector current and high-injection effects ......28

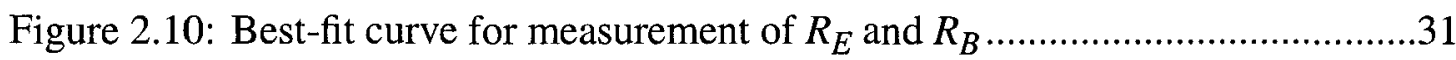

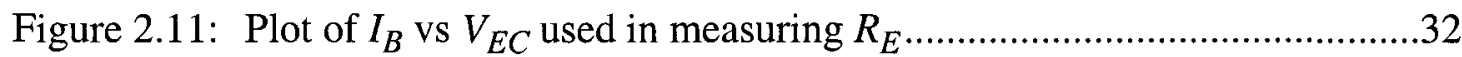

Figure 2.12: Lateral PNP minority charge distribution model .....................................34

Figure 2.13: Initial DC characteristic curves for lateral PNP SPICE model................40

Figure 2.14: Optimized DC characteristic curves for lateral PNP SPICE model .......41

Figure 2.15: Linear plot of characteristic curves for lateral PNP SPICE model .........42

\section{Chapter 3: The Design of a Log-Domain Integrator in}

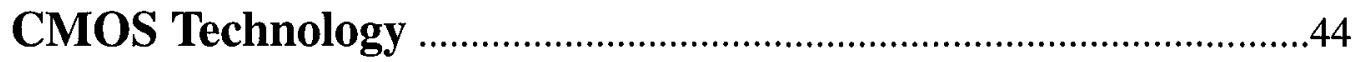

Figure 3.1: The basic two-transistor log-domain cell...........................................45

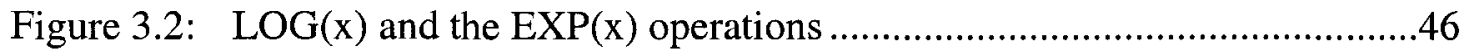

Figure 3.3: A PNP-only log-domain integrator ..................................................48

Figure 3.4: Symbolic representation of an integrator and damped integrator ...........50

Figure 3.5: A CMOS implementation of a log-domain integrator ........................52

Figure 3.6: A logarithmic compression and exponential expansion circuit ..............56

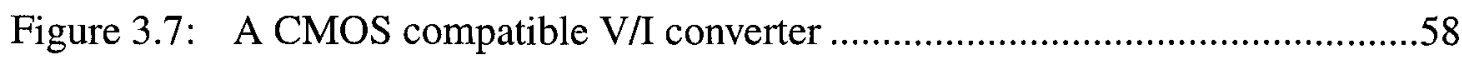


Figure 3.8: A simplified schematic of the damped integrator circuit .....................6 60

Figure 3.9: A microphotograph of the damped integrators under test ...................61

Figure 3.10: Frequency response of the log-domain integrator, $\mathrm{C}=10 \mathrm{pF}$..............62

Figure 3.11: Frequency response of the log-domain integrator, $\mathrm{I}_{\mathrm{o}}=100 \mu \mathrm{A}$..........62

Figure 3.12: Total Harmonic Distortion, $\mathrm{I}_{\mathrm{O}}=10 \mu \mathrm{A}, \mathrm{I}_{\mathrm{in}}=100 \mathrm{kHz} \ldots \ldots \ldots \ldots \ldots \ldots \ldots \ldots . . .64$

Figure 3.13: Third order intercept points, $I_{0}=10 \mu \mathrm{A}, I_{\text {in }}=100 \mathrm{kHz} \ldots \ldots \ldots \ldots \ldots \ldots . . .64$

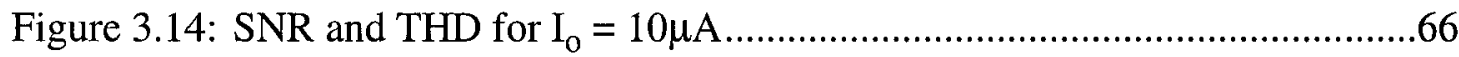

Figure 3.15: Typical output spectrum for $\mathrm{I}_{\mathrm{o}}=50 \mu \mathrm{A}, \mathrm{I}_{\mathrm{in}}=30 \mu \mathrm{A}_{\text {peak }} \ldots \ldots \ldots \ldots \ldots \ldots \ldots . .68$

\section{Chapter 4: The Design of Bipolar Log-Domain Filters in} CMOS Technology ……......................................................................

Figure 4.1: A third order low pass LC ladder based filter ..................................73

Figure 4.2: A signal flow graph for a general log-domain cell and integrator. .........74

Figure 4.3: A third order low pass filter log-domain signal flow graph ..................75

Figure 4.4: A biquadratic filter prototype, signal flow graph, implementation........78

Figure 4.5: A simplified schematic of the final biquadratic filter implementation....79

Figure 4.6: An elliptic LC filter prototype, signal flow graph, implementation.......82

Figure 4.7: A simplified schematic of the final elliptic filter implementation..........83

Figure 4.8: A microphotograph of the biquadratic and elliptic filter .......................86

Figure 4.9: Frequency response of biquadratic filter \# $1, \mathrm{I}_{\mathrm{O}}=1 \mu \mathrm{A}=100 \mu \mathrm{A}$........87

Figure 4.10: Frequency response of biquadratic filter \# 1 - \# $3, \mathrm{I}_{0}=50 \mu \mathrm{A} \ldots \ldots \ldots \ldots . .87$

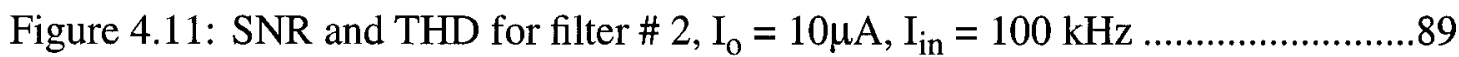

Figure 4.12: Third order intercept points for filter \#1, $\mathrm{I}_{\mathrm{o}}=10 \mu \mathrm{A}, \mathrm{I}_{\mathrm{in}}=100 \mathrm{kHz} \ldots \ldots .89$

Figure 4.13: Frequency response of elliptic filter \# 4 - \# 6, $\mathrm{I}_{\mathrm{O}}=10 \mu \mathrm{A} \ldots \ldots \ldots \ldots \ldots \ldots . . . . . . . .92$

Figure 4.14: Frequency response of elliptic filter \# 4 - \# 6, $\mathrm{I}_{\mathrm{o}}=100 \mu \mathrm{A} \ldots \ldots \ldots \ldots \ldots . . . . . .92$

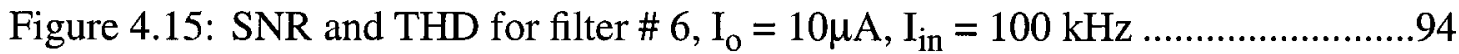

Figure 4.16: Third order intercept points for filter \# $6, \mathrm{I}_{\mathrm{o}}=10 \mu \mathrm{A}, \mathrm{I}_{\text {in }}=100 \mathrm{kHz} \ldots . .94$ 


\section{List of Tables}

Chapter 1: Introduction 1

\section{Chapter 2: Modelling Lateral PNP Transistors in}

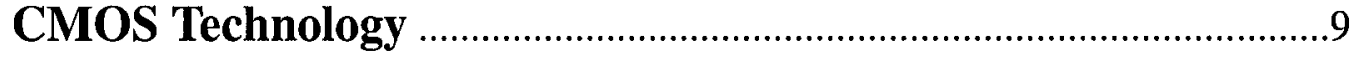

Table 2.1: SPICE parameter listing ………………….........................................13

Table 2.2: Parasitic Capacitance Parameters for the SPICE model .........................38

Table 2.3: Initial values for SPICE lateral PNP model .......................................40

Table 2.4: Optimized values for SPICE lateral PNP model ....................................41

\section{Chapter 3: The Design of a Log-Domain Integrator in}

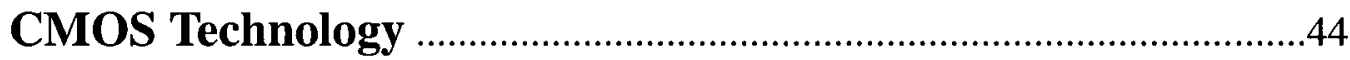

Table 3.1: Transistor dimensions for CMOS log-domain integrator circuit ...........54

Table 3.2: Transistor dimensions for compression and expansion circuits .............57

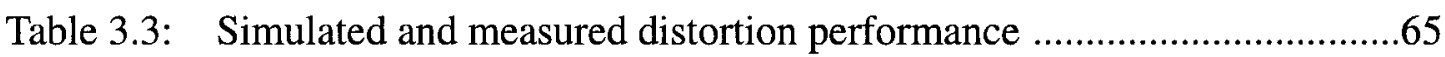

Table 3.4: Simulated and measured noise performance ………………...................67

Table 3.5: A summary of the damped integrator performance ...............................69

\section{Chapter 4: The Design of Bipolar Log-Domain Filters in}

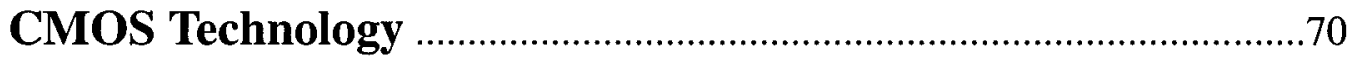

Table 4.1: Capacitor values used for biquadratic and elliptic filter testing ..............85

Table 4.2: A summary of the biquadratic filter performance ..................................90

Table 4.3: A summary of elliptic filter performance ..............................................95

Table 4.4: Comparison of original bipolar and CMOS filter performance ..............96

Table 4.5: Comparison of experimental CMOS filter performance ........................98

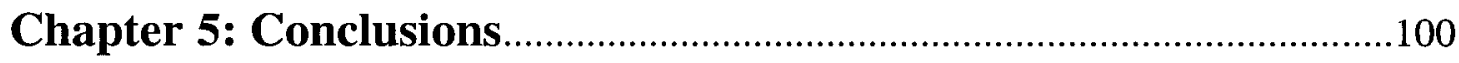

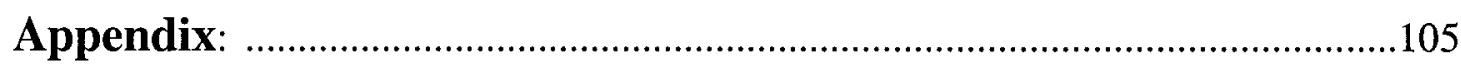

Table A.1 - Parameter values for the lateral PNP transistor in 0.35 $\mu$ CMOS ...........105 


\section{Chapter 1 - Introduction}

\section{1 - Motivation}

Log-domain filters have emerged in recent years as a new and important class of continuous-time filter. Unlike conventional classes of filters, in which linear circuits are implemented using non-linear devices, log-domain filters directly employ a transistor's non-linear (in this case, logarithmic) behaviour. Without the need for conventional circuit linearization techniques, log-domain filter circuits have a simple and elegant structure, and hold potential to run at high frequencies and operate from low power supplies. Logdomain filters also possess many other attractive features, including the ability to be electronically tuned over a wide range of frequencies. In addition, as is the case with all companding filters, log-domain filters are theoretically capable of obtaining very large dynamic ranges [1].

At the heart of the log domain filtering technique is the logarithmic / exponential relationship between voltage and current in a transistor - traditionally, a bipolar transistor. For economic and system-integration reasons, efforts are underway to extend the logdomain approach from bipolar to CMOS technology. Most research on this topic has focused on the use of the MOS transistor operating in weak inversion. Though such devices exhibit the desired logarithmic / exponential behaviour, the relatively low operating currents place limitations on the bandwidth which can reasonably be achieved. 
In addition, the modelling of subthreshold characteristics in commercial CMOS processes is often insufficient for high performance applications.

An alternate approach to the implementation of log domain filters in CMOS technology, and one which avoids the low current and low bandwidth limitations of subthreshold operation, is to design filters using the lateral bipolar device inherent in standard CMOS processes. While the characteristics of the lateral bipolar transistor have traditionally lagged far behind the vertical transistor, the continually decreasing dimensions of CMOS technology have made the lateral device increasingly more attractive. The development of log-domain filters in CMOS technology using lateral bipolar transistors shall be the subject of this dissertation.

The outline of this chapter is as follows: the first section will provide a description of the evolution of the log domain filter as it pertains to this work. The next section will present an introduction to the concepts of log domain filtering, and will provide an overview of a log-domain filter structure and implementation. The final section will discuss the organization of this thesis and provide an outline of its contents. A brief historical background shall be provided to begin.

\section{2 - A Historical Background}

The concept of the log-domain filter was originally proposed by Adams and introduced to the Audio Engineering Society in 1979 [2]. Adams had developed a method by which the resistor in an RC filter could effectively be replaced with a diode, a nonlinear element. By controlling the bias current of the diode, the cutoff frequency of the filter could be electronically tuned over several decades. Adams described the discovery as "a circuit, composed of both linear and non-linear elements, which, when placed between a $\log$ converter and an anti-log converter (in the "log domain"), will cause the system to act as a linear filter".

The technique introduced by Adams did not receive significant attention until 1993, when Frey [3] proposed a formal procedure for synthesizing log-domain filter 
functions. The method developed by Frey introduced state-space matrices as a means to describe the operation of the filter. Frey demonstrated the technique with the design of a biquadratic log-domain filter and a seventh order Chebychev filter, implemented as a cascade of biquadratic stages.

A more intuitive procedure for synthesizing log-domain filter functions was proposed by Perry and Roberts [4], [5] in 1995. The method introduced signal-flow graphs as a means to describe the operation of the filter, from which common filter design techniques, such as the operational simulation of LC ladders [6], could be applied. Such techniques can be used to simplify the filter design procedure, and can readily be extended to high-order systems. At the heart of this filter design approach is a circuit referred to as an integrator, or in this case, a "log-domain" integrator. Note that the signal flow graph, the method of operational simulation, and the concept and design of the log-domain integrator shall be described in detail and will figure prominently throughout this work.

Numerous implementations of bipolar log-domain integrators have been described in the literature. One of the earliest such integrators was introduced by Seevinck [7] in 1990. Though developed independently of the work by Adams or Frey, Seevinck described "a companding current-mode integrator", a circuit which performed logarithmic compression on input signals and exponential expansions on output signals, yet overall performed a linear integration, conforming very closely to the paradigm of a log-domain filter. Subsequently, many integrator implementations have been described in the literature, and several are described in detail in [1]. Of particular interest will be an NPNonly, low voltage integrator introduced by El-Gamal and Roberts [8], which shall form the basis for the integrator developed in Chapter 3 of this work.

The performance of high-order log-domain filters has been well reported in the literature. Among them, Frey has reported a high-frequency second-order bandpass filter [9], shown to be capable of operation at over $400 \mathrm{MHz}$. A low-power third-order BiCMOS filter, reported by Punzenberger and Enz [10], has been shown to be capable of being tuned over many decades of frequency, with a maximum frequency of about $10 \mathrm{MHz}$, while operating from a 1.2 V supply. The NPN-only integrator mentioned in the previous paragraph has been 
used by El-Gamal et. al. [11] in the design of a high-frequency third-order filter, shown to be capable of operation at up to $100 \mathrm{MHz}$ while operating from a $1.2 \mathrm{~V}$ supply.

Of particular interest in this thesis is the implementation of log-domain filters in CMOS technology. One of the first such implementations was reported by Toumazou et. al. [12] in 1994. In this work, Toumazou described a second order low-pass filter which made use of MOSFETs operating in weak inversion, intended for use in a micropower biomedical application. More recently, many papers have been devoted to the subject of CMOS log domain filters. Those which provide an experimental verification of their findings, however, are relatively few in number [13]-[19]. A comparison of the experimental results in previously published papers with respect to the filter performance attained in this work will be provided at the end of Chapter 4.

The vast majority of log-domain research in CMOS technology to this point has focused on the use of the MOS transistor operating in its subthreshold region [12]-[18], and therefore this research has been confined to low power and relatively low frequency implementations. Attempts have been made to extend the concepts of log-domain filters to CMOS circuits according to the square law relationship of a MOSFET transistor operating in saturation. Such an approach was proposed by Frey [20] in 2000. Although this approach may have merits, the methods involved are not straightforward. Circuits for implementing such relationships have been proposed [20], [21] though to date no experimental implementations of these filters have been reported in the literature, and no information on the simulated linearity and distortion of such filters has been provided.

Rather than making use of MOSFET transistors in weak inversion or developing a new system for implementing square law filters, an alternate approach to implementing $\log$ domain filters in CMOS technology is to make use of the lateral bipolar device inherently available within the CMOS process. The development of this approach shall be the subject of the subsequent chapters of this work. 


\section{3 - An Overview of Log-Domain Filtering}

A simple explanation of the principle of log domain filtering can be understood in terms of the diode - capacitor circuit first described by Adams, as shown in Figure 1.1. If one assumes that the current flowing through the diode can be expressed by a simple exponential function, the differential equation describing the operation of this circuit could be expressed as

$$
e^{\left(V_{I}-V_{o}\right)}=C \frac{d}{d t}\left(V_{o}\right)
$$

which can be rewritten as

$$
e^{V_{I}}=C \frac{d}{d t}\left(e^{V_{o}}\right)
$$

or written in integral form as

$$
e^{V_{o}}=\frac{1}{C} \int e^{V_{I}} d t .
$$

This circuit does not implement a linear integrator in terms of the input and output voltages $V_{I}$ and $V_{O}$. However, if these variables were replaced by $X_{I}$ and $X_{O}$, according to

$$
V_{I}=\ln \left(X_{I}\right), \quad V_{O}=\ln \left(X_{O}\right),
$$

then Eqn. (1.3) could be rewritten as

$$
X_{O}=\frac{1}{C} \int X_{I} d t
$$

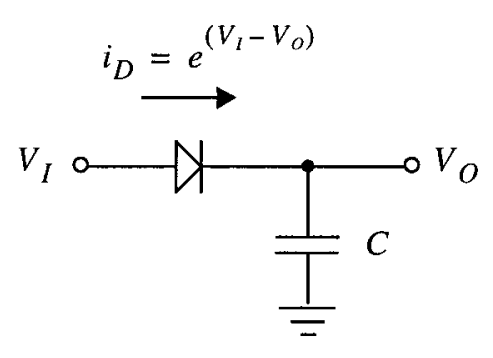

Figure 1.1 A simple log-domain integrator 
Therefore, by logarithmically compressing the signal $X_{I}$ according to Eqn. (1.4) to obtain $V_{I}$ and the circuit input and by exponentially expanding $V_{O}$ according to the inverse of Eqn. (1.4) to obtain $X_{O}$ at the circuit output, a linear integration can be implemented. Such a means of expansion and compression is readily available in the form of the voltage to current relationship of the diode equation itself. In this case, the desired input and output signals, $X_{I}$ and $X_{O}$, are in fact currents, and the integration which is taking place is performed on logarithmically compressed signals, or put another way, in the "logarithmic domain".

Though the advantages of doing so are not immediately obvious, it can be shown that the constant of integration (not included in the previous analysis) can be designed to be proportional to the bias current in the diode. Therefore, the cut-off frequencies of filters designed with such an integrator can be electronically tuned, in this particular case over a range of many decades. In addition, the capacitor storage element for this integrator is subject to signals which have undergone logarithmic compression, and therefore the theoretical dynamic range of the integrator can be very large. Furthermore, this system requires no feedback in order to linearize the response of its components; rather, the inherent non-linearity of its elements are used to its advantage. Each of these features make this simple integrator both intriguing and potentially very useful.

In Chapter 3, a more rigorous mathematical approach to the development of a logdomain will be presented, though the underlying principles will remain the same. Rather than a diode, a bipolar PNP transistor, with a logarithmic / exponential voltage to current relationship given by

$$
I_{C}=I_{S} e^{V_{B E} / v_{T}}
$$

will be used to implement the integrator to be used in this work.

A generic block diagram for a log domain filter is shown in Figure 1.2. This filter is composed of three distinct sections: an input compression stage, a filter stage, and an output expansion stage. Voltage to current conversion and logarithmic compression are 


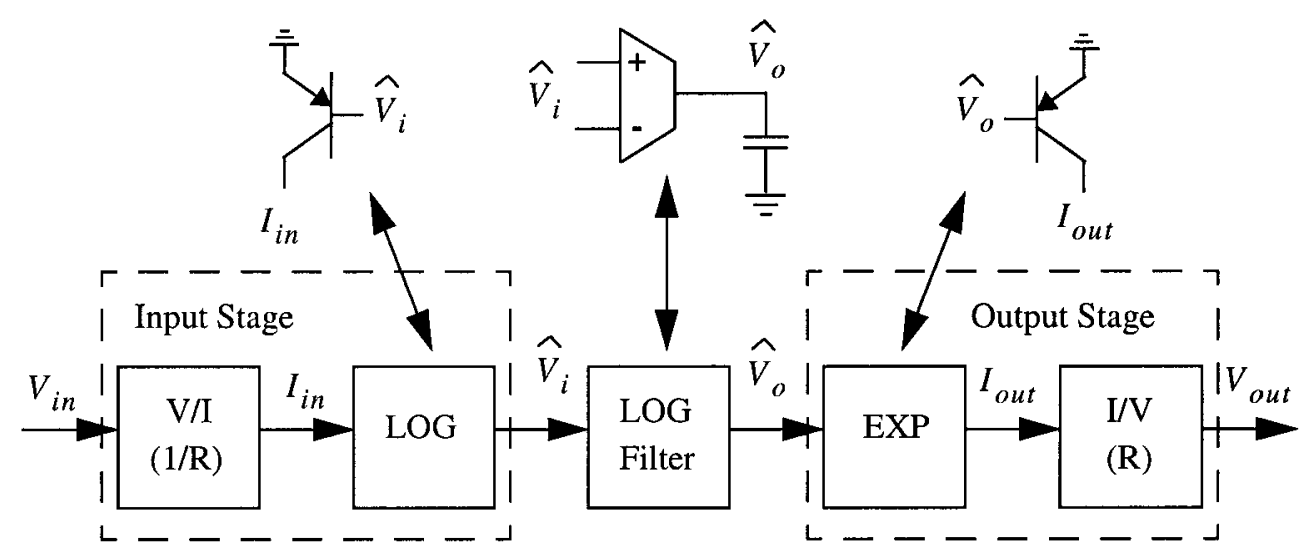

Figure 1.2 Block diagram of a log-domain filter

performed in the first stage, as shown by the progression of the $V_{i n}, I_{i n}$, and $\hat{V}_{i}$ signals in the figure. The $\mathrm{V} / \mathrm{I}$ conversion ratio is represented by $1 / R$ in the figure, and the logarithmic compression is performed by means of a bipolar transistor. Filtering (in the logarithmic domain) is performed in the second stage. The symbol for the log-domain integrator, the basic building block for the filter, is drawn in the figure. Exponential expansion and I/V conversion are performed in the final stage, as shown by the progression of $\hat{V}_{o}, I_{\text {out }}$, and $V_{\text {out }}$ signals. Exponential expansion is once again performed by means of a bipolar transistor, and the $\mathrm{I} / \mathrm{V}$ conversion ratio is represented in the figure by $R$. Each of the blocks shown above will be described in detail in this work.

\section{4 - Thesis Outline}

The development of log-domain filters in CMOS technology using lateral bipolar transistors shall be the subject of this dissertation. The presentation in this work will be organized according to three distinct levels of hierarchy. The first of the three shall be an investigation at a device level, where a characterization of the lateral PNP transistor will be performed. The second shall be an investigation at a circuit level, where the design of a log-domain integrator and associated circuitry will be developed. The third shall be an investigation at a system level, where the implementation of an entire log-domain filter will be described in detail. 
Following this outline, Chapter 2 will describe the characterization of a lateral PNP transistor in a standard $0.35 \mu$ CMOS process. The structure and layout of the transistor will be presented, and a SPICE-compatible circuit model for the device will be developed. Techniques for determining the device parameters and physical insights into the device operation shall be described, and a comparison of the modelled and measured low frequency response of the transistor shall be provided.

Chapter 3 will describe the development of a log-domain integrator which makes use of these lateral PNP devices. The theoretical background required to understand the operation of a log-domain integrator will be provided and the methodology of mapping the log-domain integrator from bipolar into CMOS technology will be presented. The simulated and experimentally measured integrator response shall be compared, demonstrating some of the capabilities and limitations of this new log-domain filtering approach.

Chapter 4 shall describe the complete implementation of an log-domain filter. The theoretical background required to synthesize second and higher-order filters, based upon on the log-domain integrator, will be provided. The filter synthesis, complete circuit implementation, and experimental characterization of a biqudratic and a third-order elliptic filter will be performed. A comparison of the characteristics of the CMOS logdomain filters developed in this work to other log-domain filter implementations described in the literature will be provided.

As a result of this work presented in these chapters, a first, second, and third order $\log$ domain filter will have been thoroughly investigated. The final chapter shall provide conclusions which may be drawn from this work, and will discuss a few future directions for log-domain circuit design in CMOS technology. 


\section{Chapter 2 - Modelling Lateral PNP}

\section{Transistors in CMOS}

\section{Technology}

In order to realize log-domain circuits in CMOS technology, a logarithmicexponential device such as a bipolar transistor is required. The lateral bipolar transistor, which may be fabricated in a standard CMOS process, can be used for this purpose. However, this device is not in widespread use in CMOS circuit design, and transistor models for the lateral bipolar device are generally not provided by semiconductor manufacturers. In this chapter, a SPICE compatible model for a lateral PNP transistor, fabricated in $0.35 \mu$ CMOS technology, will be presented.

Keeping the lateral PNP model as simple as possible has been one of the key motivations of this work. Therefore, the number of parameters used to describe the device behaviour has been kept to a minimum. Furthermore, each of the parameters can be either determined from physical considerations, extracted from straightforward device measurements, or adopted from standard information provided by the manufacturer. The model can therefore be readily developed within any CMOS process. 
The outline of this chapter is as follows: the first section will describe the lateral PNP transistor structure and will discuss several device layout considerations. The second section will describe each of the SPICE parameters used in modelling the transistor, and will provide both physical insights into the device operation and techniques for extracting the parameters from measurements where applicable. The final section of the chapter will provide the complete SPICE model for the transistor. The model will form the basis for all the designs and simulations of log-domain filter circuits described in this work.

\section{1 - Lateral PNP Transistors: Structure and Layout}

In standard n-well CMOS technology, two types of bipolar devices can be fabricated: a vertical PNP transistor and a lateral PNP transistor. A cross sectional diagram of each type of device is shown in Figure 2.1. Note that in an n-well technology, only PNP devices, rather than NPN devices, can be formed.

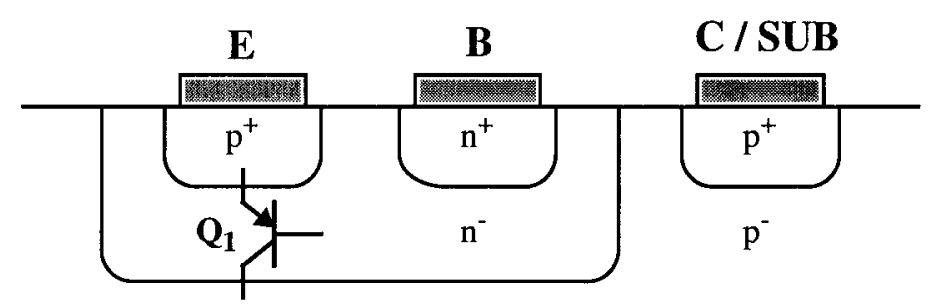

(a)

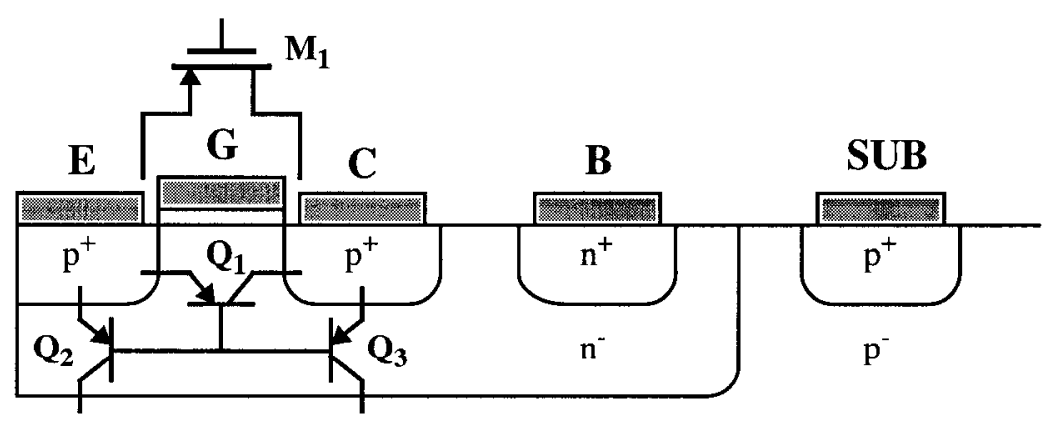

(b)

Figure 2.1 (a) Cross section of a vertical PNP transistor

(b) Cross section of a lateral PNP transistor 
The vertical PNP transistor is formed using a $\mathrm{p}^{+}$diffusion for the emitter, an n-well for the base, and the $\mathrm{p}^{-}$substrate for the collector, as indicated by $\mathrm{Q}_{1}$ in Figure 2.1(a). The structure is straightforward, and models for vertical PNP transistors are generally provided by the semiconductor manufacturer. These devices, however, are of limited use for most analog applications since the collector coincides with the substrate of the chip.

The lateral PNP transistor is formed using the structure of a PMOS transistor. The $\mathrm{p}^{+}$diffusions of the source and drain of the PMOS transistor are used to form the emitter and collector of the device, while the lightly doped n-well region under the gate of the PMOS transistor is used to form the base of the lateral device, as indicated by $Q_{1}$ in Figure 2.1(b). However, by the nature of this structure, three undesired devices are also present: two other parasitic vertical bipolar transistors $\left(Q_{2}-Q_{3}\right)$, and the original PMOS transistor $\left(\mathrm{M}_{1}\right)$ used in forming this lateral device.

The model of the behaviour can be simplified if the structure is operated with the gate terminal connected to a sufficiently large positive potential, thereby ensuring that the PMOS transistor $\left(\mathrm{M}_{1}\right)$ is off. The modelling can be further simplified by observing that the contribution of the second parasitic vertical bipolar transistor $\left(\mathrm{Q}_{4}\right)$ will be minimal if the lateral bipolar device $\left(\mathrm{Q}_{2}\right)$ is kept from operating deeply into saturation. The behaviour of this structure can therefore be modelled adequately by including only the two remaining devices, shown schematically in Figure 2.2(a) and symbolically in Figure 2.2(b). This two transistor configuration will form the basis for the lateral PNP model described in this chapter.

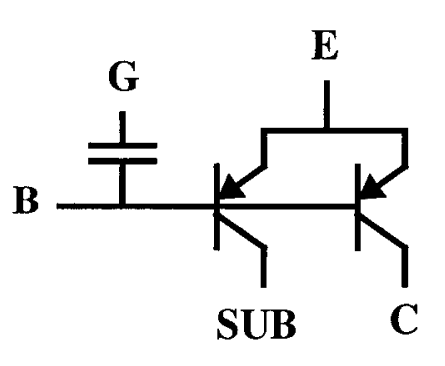

(a)

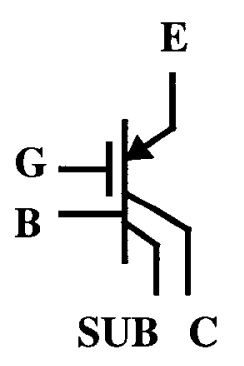

(b)

Figure 2.2 (a) Schematic and (b) Symbolic representation of a lateral PNP device 
Before discussing the specifics of the modelling, several layout considerations should be mentioned. First, lateral PNP transistors are conventionally laid out in circular fashion, with the emitter at the centre. This improves the injection efficiency, since the emitter is entirely enclosed by the collector, and serves to keep the injection uniformly distributed, since the device is symmetric. Secondly, the lateral emitter injection is, to a first approximation, proportional to the emitter perimeter, which scales with the diameter, while the vertical emitter injection is, to a first approximation, proportional to the emitter area, which scales with the square of the diameter. To maximize the ratio of lateral to vertical collector current, the diameter of the emitter should therefore be kept to a minimum. Finally, note that base width of the lateral device is set by the gate length of the original PMOS transistor. In order to maximize the current gain of the lateral device, the gate length should be kept to a minimum. The layout of a typical lateral PNP device, fabricated in $0.35 \mu$ CMOS technology, is shown in Figure 2.3.

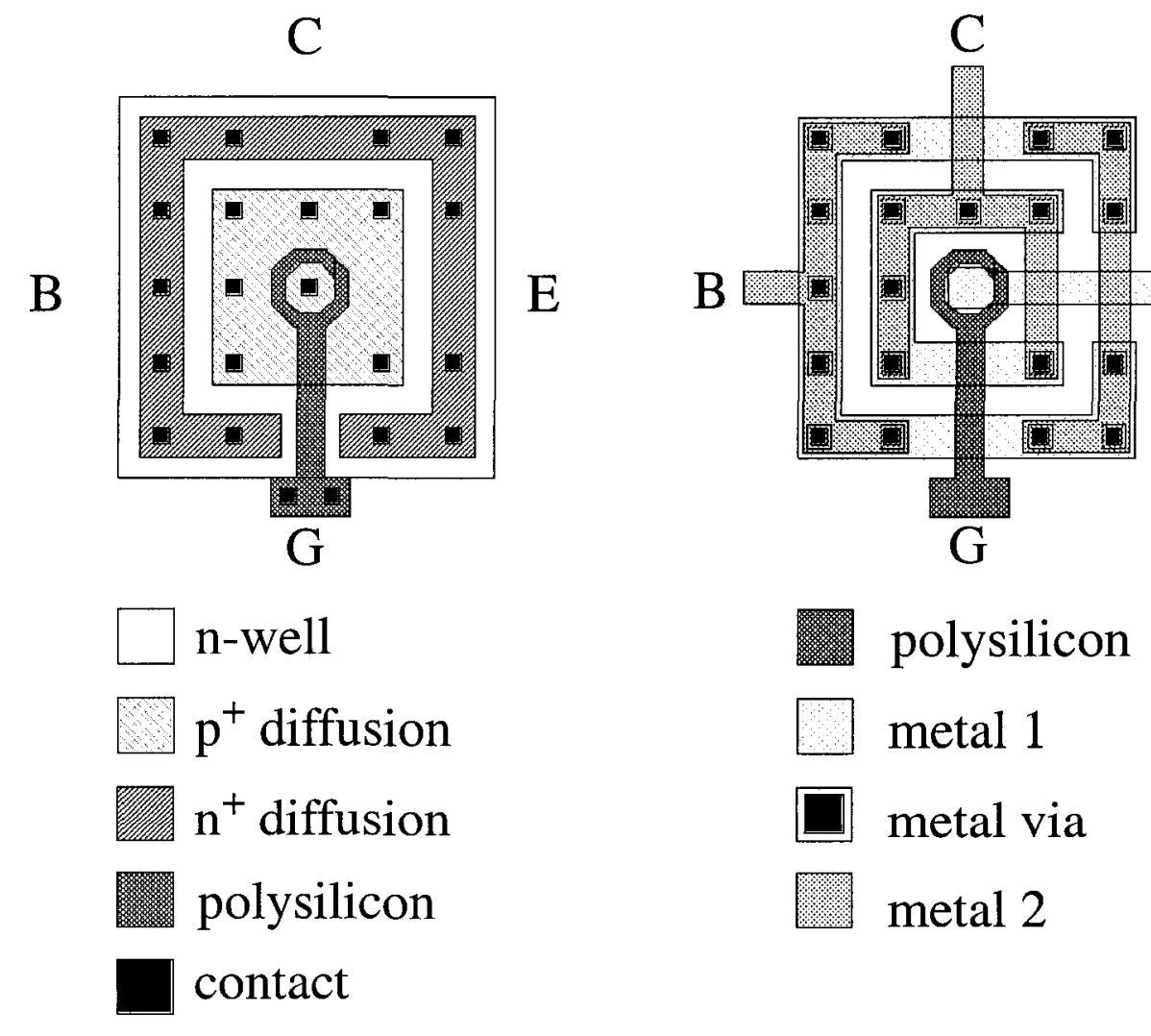

Figure 2.3 Layout of a minimum size 0.35 $\mu$ CMOS lateral PNP transistor 


\section{2 - Lateral PNP Transistors: SPICE Model Parameters}

A two-transistor SPICE compatible model as shown in Figure 2.2 has been developed for the $0.35 \mu$ CMOS lateral PNP transistor as shown in Figure 2.3. The number of parameters used to describe the device behaviour has been kept to a minimum (a total of fourteen SPICE parameters and one additional capacitance) with the intention that the model should be sufficiently simple to be readily developed in any CMOS process. Low frequency parameters have been measured or fitted directly, while AC parameters and parasitic parameters have been adapted from existing modelling information provided by the manufacturer. A listing of each of the SPICE parameters used in developing this model are given in Table 2.1. A detailed description of these parameters can be found in several references, including [22] and [23]. Previous work in developing SPICE models for lateral bipolar transistors can be found in [24] and [25].

Table 2.1:SPICE Parameter Listing

\begin{tabular}{|c|l|}
\hline $\begin{array}{c}\text { SPICE } \\
\text { Parameter }\end{array}$ & \multicolumn{1}{c|}{$\begin{array}{c}\text { Parameter Description } \\
(\text { Common Usage Name in Brackets })\end{array}$} \\
\hline IS & Saturation Current $\left(\mathrm{I}_{\mathrm{s}}\right)$ \\
\hline NF & Forward Emission Coefficient $\left(\mathrm{n}_{\mathrm{F}}\right)$ \\
\hline BF & Maximum Forward Current Gain $(\beta)$ \\
\hline VAF & Forward Early Voltage $\left(\mathrm{V}_{\mathrm{A}}\right)$ \\
\hline $\mathrm{IKF}$ & Forward Knee Current $\left(\mathrm{I}_{\mathrm{KF}}\right)$ \\
\hline $\mathrm{RE}$ & Ohmic Emitter Resistance $\left(\mathrm{R}_{\mathrm{E}}\right)$ \\
\hline $\mathrm{RB}$ & Ohmic Base Resistance $\left(\mathrm{R}_{\mathrm{B}}\right)$ \\
\hline $\mathrm{TF}$ & Forward Base Transit Time $\left(\tau_{\mathrm{F}}\right)$ \\
\hline $\mathrm{CJE}$ & Zero Bias Base-Emitter Junction Capacitance $\left(\mathrm{C}_{\mathrm{je}}\right)$ \\
\hline VJE & Base-Emitter Junction Built-In Potential $\left(\phi_{\mathrm{je}}\right)$ \\
\hline MJE & Base-Emitter Junction Grading Coefficient $\left(\mathrm{m}_{\mathrm{je}}\right)$ \\
\hline CJC & Zero Bias Base-Collector Junction Capacitance $\left(\mathrm{C}_{\mathrm{jc}}\right)$ \\
\hline VJC & Base-Collector Junction Built-In Potential $\left(\phi_{\mathrm{jc}}\right)$ \\
\hline MJC & Base-Collector Junction Grading Coefficient $\left(\mathrm{m}_{\mathrm{je}}\right)$ \\
\hline
\end{tabular}


One of the most significant complications in developing a model for the lateral PNP transistor stems from the fact that the lateral and vertical devices, which share emitter and base regions, do not operate independently of one another, as would be assumed by the two transistor model presented in Figure 2.2(a). This problem manifests itself in two ways. First, when making transistor measurements, the parameters of one device may depend on the bias conditions of the other device. For example, the vertical collector current has been observed to have a dependence on the voltage bias of the lateral collector-base junction. Secondly, when measuring certain device quantities, the contribution of the lateral and vertical devices cannot be distinguished from one another. For example, the base currents of the lateral and vertical devices cannot be separated, since they are connected though one common terminal. To alleviate the first of these problems, the transistors have been modelled using bias conditions which most closely resemble those under which the devices will operate in log-domain filter applications. To alleviate the second problem, reasonable approximations have been made when quantities cannot be directly measured. Comparison of measured values with first order physically-based models have been used for this purpose whenever possible.

Another significant complication in developing a model for the lateral PNP transistor stems from the relatively light doping of the n-well in this $0.35 \mu$ CMOS process. The doping concentration of the n-well, which is used to form the base of the transistor, on the order of $5 \times 10^{16} \mathrm{~cm}^{-3}$ (see the listing in the Appendix), which is approximately a factor of ten lower than would be the case in a standard bipolar process [26]. The light doping in the base affects the transistor behaviour in several ways, and will be given particular attention as the transistor model is presented.

The following sections of this chapter will describe the SPICE model parameters of Table 2.1, including the physical origins of each parameter as well as the parameter measurement techniques where applicable. The theoretical background presented this chapter has been compiled from [22], [26]-[29]. Additional information on lateral bipolar transistors, including detailed discussions of the devices physics, can be found in [24], [30] and [31].

Each of the parameter values developed in this section will be used in a final model optimization, which will be described in Section 2.3 at the end of the chapter. 


\subsection{1 - Saturation Current $\left(I_{S}\right)$}

The saturation current $\left(I_{S}\right)$ is one of the most fundamental parameters in the operation of a bipolar transistor. For a PNP device, the collector current is determined from $I_{S}$ according to

$$
I_{C}=I_{S} \cdot e^{\frac{V_{E B}}{v_{T}}}
$$

where $I_{C}$ represents the collector current, $V_{E B}$ represents the emitter-base voltage, and $v_{T}$ represents the thermal voltage. This section of the chapter will provide a brief theoretical derivation of the saturation current (which will be referenced throughout the chapter) and will present a comparison between the theoretically modelled and experimentally measured collector currents for the lateral PNP devices.

A first order model of collector current can be developed by assuming that the current arises entirely from the diffusion of holes (the minority carrier) through the base from the emitter to the collector of the device. This diffusion current can be expressed as

$$
i_{p}=q A_{E B} D_{p} \frac{d p_{n}}{d x}
$$

where $i_{p}$ represents the hole diffusion current, $q$ represents the charge of an electron, $A_{E B}$ represents the effective area of the emitter-base junction, $D_{p}$ represents the diffusion constant for holes in the base, and $\frac{d p_{n}}{d x}$ represents the gradient of the concentration of holes in the direction from the emitter to the collector.

If one assumes that negligible carrier recombination occurs in the base (a valid assumption given the light base doping of these devices) the concentration of holes can be approximated as varying uniformly between the emitter-base junction and the basecollector junction. The gradient of the concentration can therefore be expressed as

$$
\frac{d p_{n}}{d x}=\frac{p_{n(e b j)}-p_{n(b c j)}}{W_{B}} \approx \frac{p_{n(e b j)}}{W_{B}}
$$


where $p_{n(e b j)}$ and $p_{n(b c j)}$ represent the hole concentration on the n-doped side of the emitter-base junction and collector-base junction respectively, and $W_{B}$ represents the effective width of the base. Under normal operating conditions, the concentration of holes at the reversed biased base-collector junction is approximately zero, and therefore $\frac{d p_{n}}{d x}$ is directly proportional only to the concentration of holes in the base at the emitter-base junction, as indicated by the approximation in Eqn. (2.3).

For low to moderate levels of emitter-base voltage, the concentration of holes at the emitter-base junction will equal the rate of injection of holes from the emitter, given by

$$
p_{n(\mathrm{ebj})}=p_{n o} \cdot e^{\frac{V_{E B}}{v_{T}}}
$$

where $p_{n o}$ represents the equilibrium hole concentration in n-doped base, $V_{E B}$ represents the forward bias placed across the emitter-base junction, and $v_{T}$ represents the thermal voltage. Since holes are the minority carrier, the hole concentration can be expressed in terms of the majority carrier concentration, which at low levels of injection is equal to the doping concentration of the base. Therefore $p_{n o}$ can be replaced by $n_{i}^{2} / N_{B}$, where $n_{i}$ is the intrinsic carrier concentration of silicon and $N_{B}$ represents the doping concentration in the base. The collector current can therefore be expressed by combing equations (2.2), (2.3), and (2.4) to give

$$
I_{C}=\frac{q A_{E B} D_{p} n_{i}^{2}}{N_{B} W_{B}} \cdot e^{\frac{V_{E B}}{v_{T}}}=I_{S} \cdot e^{\frac{V_{E B}}{v_{T}}}
$$

where the saturation current, $I_{S}$, is defined according to Eqn. (2.5), as is found in several references [27]-[26]. This parameter is also often defined as the value of collector current when $V_{E B}$ is set equal to zero.

From this definition, it is possible to estimate a theoretical value of $I_{S}$ for both the lateral and vertical PNP transistors present in our model. Using the dimensions and physical constants tabulated in the Appendix, the theoretical value of $I_{S}$ can be calculated to be $2.09 \cdot 10^{-18} \mathrm{~A}$ for the lateral device and $7.53 \cdot 10^{-19} \mathrm{~A}$ for the vertical device. 
A comparison between the theoretically modelled and experimentally measured collector currents is shown in Figure 2.4. All measurements have been made with $V_{C B}$ fixed at $0 \mathrm{~V}$, the gate to emitter voltage, $V_{G E}$, set to $+0.5 \mathrm{~V}$, and the substrate voltage, $V_{S U B}$, set to $-2 \mathrm{~V}$ below the emitter voltage. This biasing arrangement most closely resembles the conditions under which the device will be operated in the intended log-domain filter applications. The experimental values shown here represent the average of six measurements: two independent sets of measurements each from three lateral bipolar devices, each device originating in a different silicon chip.

As is shown, there are significant deviations between the modelled and measured collector currents. The decrease in collector current for large values of base-emitter forward bias can be attributed to high-injection effects and parasitic base and emitter resistance. These effects will be addressed and modelled in Section 2.2 .5 and Section 2.2.6. There are also significant deviations in lateral collector current at low values of base-emitter voltage, and in particular, there is a variation in the slope between the modelled and measured lateral collector current curves. This effect is difficult to model theoretically, and has been accounted for empirically using the forward emission coefficient, $n_{F}$, which will be discussed in the next section.

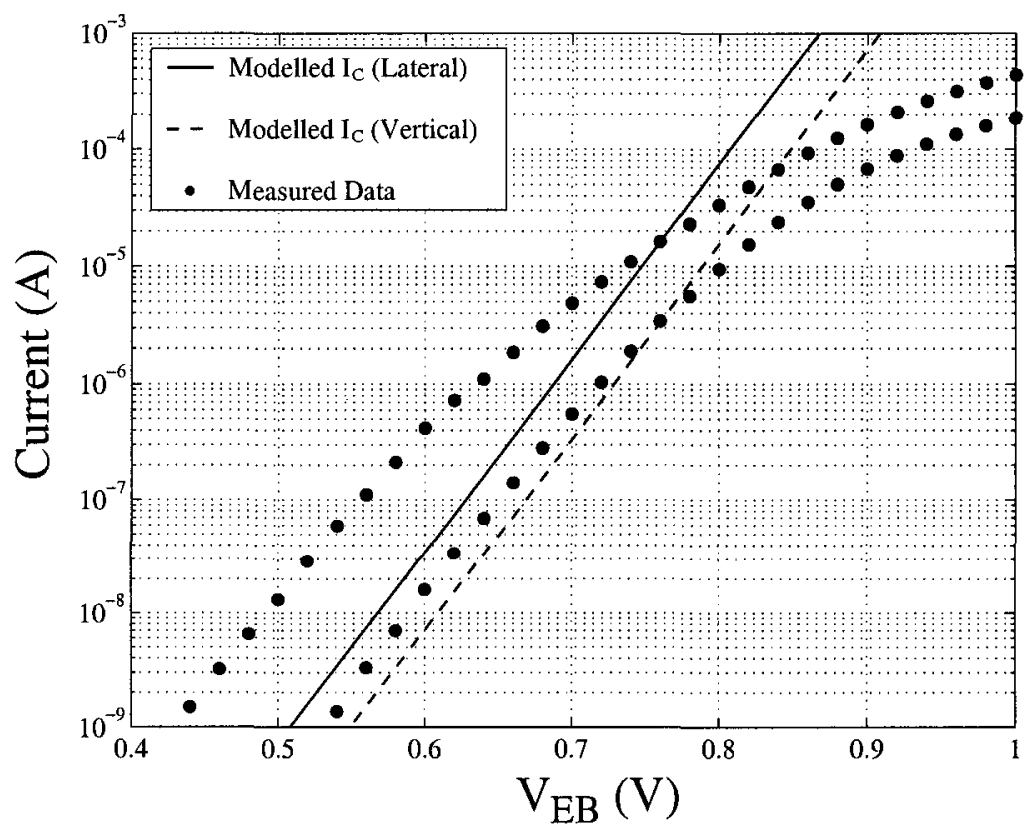

Figure 2.4 Comparison of Eqn. (2.5) and experimentally measured collector currents 


\subsection{2 - Forward Emission Coefficient $\left(n_{F}\right)$}

In order to compensate for the difference between the theoretical and experimental collector currents at low values of base-emitter forward bias, a second parameter, the forward emission coefficient $\left(n_{F}\right)$ needs to be introduced. The forward emission coefficient for a PNP transistor is defined in [22] and given by

$$
\frac{1}{n_{F}}=\left.\frac{v_{T}}{I_{c}} \cdot \frac{d I_{C}}{d V_{E B}}\right|_{V_{C B}=0}
$$

and is incorporated into the calculation of collector current according to

$$
I_{C}=I_{S} \cdot e^{\frac{V_{E B}}{n_{F} v_{T}}}
$$

In most modern bipolar devices, the value of $n_{F}$ is very close to unity and therefore this parameter is often omitted in device models. However, due to the relatively low doping concentration in the n-well in the $0.35 \mu$ CMOS process used here, this variable must be included. This section will provide a brief explanation of the physical mechanism which gives rise to the need to model $n_{F}$, and will describe the curve-fitting technique used to empirically determine $n_{F}$ from experimental data.

When a low forward bias is placed between the base and emitter, a depletion region exists on either side of base-emitter junction. The penetration of the depletion region into the base, $\mathrm{W}_{\mathrm{DR}}$, at a zero volt forward bias can be approximated, using equations given in [27], according to

$$
W_{\mathrm{DR}}=\left(\frac{2 \varepsilon V_{o}}{q N_{D}\left(1+N_{D} / N_{A}\right)}\right)^{1 / 2}
$$

where $V_{o}$ represents the built-in potential of the junction (approximately $0.9 \mathrm{~V}$ ), $V_{f}$ is the forward bias across the emitter-base junction, $N_{A}$ and $N_{D}$ are the doping concentrations on the emitter and base sides of the junction respectively, $\varepsilon$ represents the permittivity of silicon and $q$ represents the charge of the electron. The resulting penetration of the lateral 
emitter-base depletion region is approximately $0.12 \mu \mathrm{m}$ for a zero-volt forward bias. The penetration will approach zero as the forward bias approaches the built-in potential.

This modulation of the base, which amounts to slightly over $1 / 3$ of the entire base width, is similar, although inverse, to the Early effect (see Section 2.2.4) and has been facetiously referred to as the Late effect [22]. This modulation leads the collector current to be significantly higher than would be predicted by first order theory, specifically at low values of forward bias. This effect is difficult to model accurately, particularly with the geometries involved in these lateral devices. However, because the effect is so pronounced in this case, it must be accounted for. In general, the technique for compensating for the Late effect is to empirically model the collector current using the forward emission coefficient, $n_{F}$.

To perform an empirical fit, Eqn. (2.7) can be rearranged into the form $y=m x+b$,

$$
\ln \left(I_{C}\right)=\left(\frac{1}{n_{F} v_{T}}\right) v_{E B}+\ln \left(I_{S}\right)
$$

and values of $n_{F}$ and $I_{S}$ extracted from the slope and y-intercept of plot, respectively. Best fit curves for both lateral and vertical devices have been made using collector currents measured below $V_{E B}=0.7 \mathrm{~V}$. This was done to avoid including high injection effects which become a significant factor at approximately $V_{E B}=0.8 \mathrm{~V}$ (see Section 2.2.5). The resulting empirically calculated parameters are $I_{S}=1.20 \times 10^{-15} \mathrm{~A}$ and $n_{F}=1.19$ for the lateral device, and $I_{S}=2.75 \times 10^{-18} \mathrm{~A}$ and $n_{F}=1.03$ for vertical device. The empirically modelled collector currents, plotted with the experimentally measured currents, are shown in Figure 2.5. 


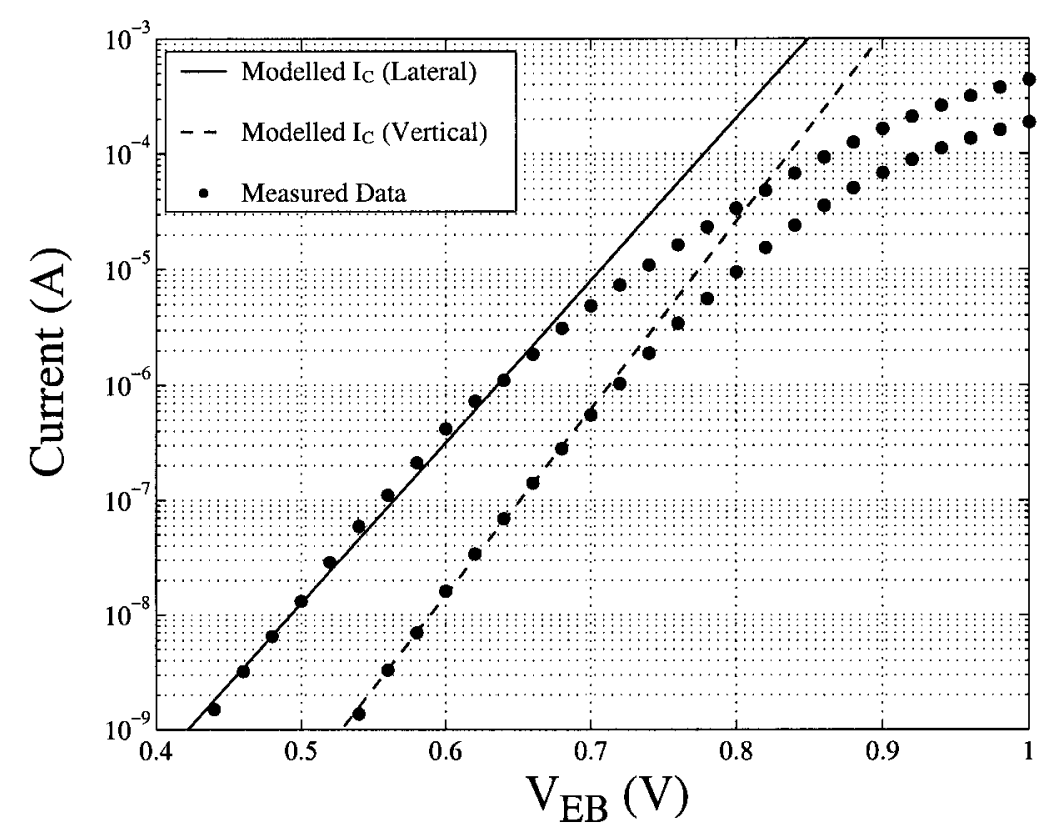

Figure 2.5 Empirical Fitting of $I_{S}$ and $n_{F}$ to experimentally measured currents

Note that the derivation given by Eqn. (2.2) to (2.5) remains a valid model for $I_{S}$. Between the regions of operation in which the Late effect and the high current injection and resistive effects dominate, Eqn. (2.5) can still be used to provide a reasonable estimate of $I_{S}$, and the derivations in Eqn. (2.2) to (2.5) will be used in later sections to derive reasonable theoretical quantities for parameters such as $I_{K F}$ and $\beta$, as described in upcoming sections. However, for the purpose of accurately capturing the collector current at low forward bias, the empirical values of $I_{S}$ and $n_{F}$ will be taken as the SPICE parameters. These parameters will be used as the starting point in the final model optimization, to be described in detail in Section 2.3. 


\subsection{3 - Forward Current Gain $(\beta)$}

The maximum forward current gain, $\beta$, is a fundamental parameter in the operation of a bipolar transistor. It is also a parameter which has been shown to have a significant impact on the performance of log-domain filters [32]. The value of $\beta$ is defined simply as ratio of collector to base current,

$$
\beta=I_{C} / I_{B} .
$$

This section will provide a derivation of the theoretical value of $\beta$ and will provide (by examining the resulting base currents) a comparison between the theoretically modelled and experimentally measured values of $\beta$ for these lateral PNP devices.

In most modern bipolar transistors, the dominant mechanism which gives rise to base current is the injection of minority carriers (electrons for a PNP transistor) from the base into emitter of the device [28]. In this case the theoretical value the base current can be derived in a similar manner to collector current, defined by Eqn. (2.2) to (2.5). The expression for base current can therefore be expressed by

$$
I_{B}=\frac{q A_{E B} D_{n} n_{i e}^{2}}{N_{E} W_{E}} \cdot e^{\frac{V_{E B}}{v_{T}}}
$$

where $N_{E}$ and $W_{E}$ are the doping concentration and effective width of the emitter, and $n_{i e}$ is the effective intrinsic concentration in the emitter.

In deriving this expression, it is important to distinguish the effective intrinsic concentration, $n_{i e}$, used in Eqn. (2.11), from the intrinsic concentration, $n_{i}$, used in Eqn. (2.5). In $0.35 \mu$ CMOS technology, the emitter is degenerately doped (that is, the doping level is sufficiently high that the Fermi level moves into the valence band for a p-type semiconductor, or into the conduction band for an n-type semiconductor). Therefore, the effective energy bandgap of a degenerately doped semiconductor is decreased by an amount $\Delta E_{g}$, leading to an effective intrinsic concentration given by 


$$
n_{i e}^{2}=n_{i}^{2} e^{\left(\frac{\Delta E_{g}}{k T}\right)}
$$

where $k$ represents Boltzman's constant and $T$ represents temperature [26]. The effective energy bandgap in the emitter is reduced by $0.125 \mathrm{eV}$, leading to an effective intrinsic concentration of $1.68 \times 10^{11} \mathrm{~cm}^{-3}$.

An expression for the theoretical value of $\beta$ can be found by combining Eqn. (2.11) with Eqn. (2.5) to give

$$
\beta=\frac{I_{C}}{I_{B}}=\frac{N_{E} W_{E} D_{p} n_{i}^{2}}{N_{B} W_{B} D_{n} n_{i e}{ }^{2}} .
$$

as stated in [28]. Using the dimensions and constants from Appendix, the theoretical value of $\beta$ can therefore be calculated to be $\beta=58$ for the lateral device and $\beta=8$ for the vertical device.

A comparison between the theoretically modelled and experimentally measured values of $\beta$ is shown in Figure 2.6. All measurements have been made with $V_{C B}=0 \mathrm{~V}$, $V_{G E}=+0.5 \mathrm{~V}$, and $V_{S U B}=-2 \mathrm{~V}$, and the measurements again represent the average of three individual devices. Since the base currents for the lateral and vertical devices cannot be independently measured in practice, the base currents shown in the figure are the sum of the contributions from both devices. Therefore, the accuracy of the theoretical value of $\beta$ is represented by the comparison of the theoretical and measured base currents.

The theoretical values of $\beta=58$ and $\beta=8$ have been used to calculate the theoretical base current, as shown by the solid line in the figure. The agreement between the theoretical and measured base currents is reasonable. An improved empirical fit, based on increasing each value of $\beta$ (while preserving the approximate ratio of lateral and vertical current gain) to $\beta=150$ and $\beta=20$, is shown by the dotted line in the figure. The increased empirical values will be used as the starting point in the final model optimization. 
Note that neither curve accounts for the decrease in collector current for large values of base-emitter forward bias. This decrease can be attributed to high-injection effects and parasitic resistances, and will be addressed in Section 2.2.5 and Section 2.2.6.

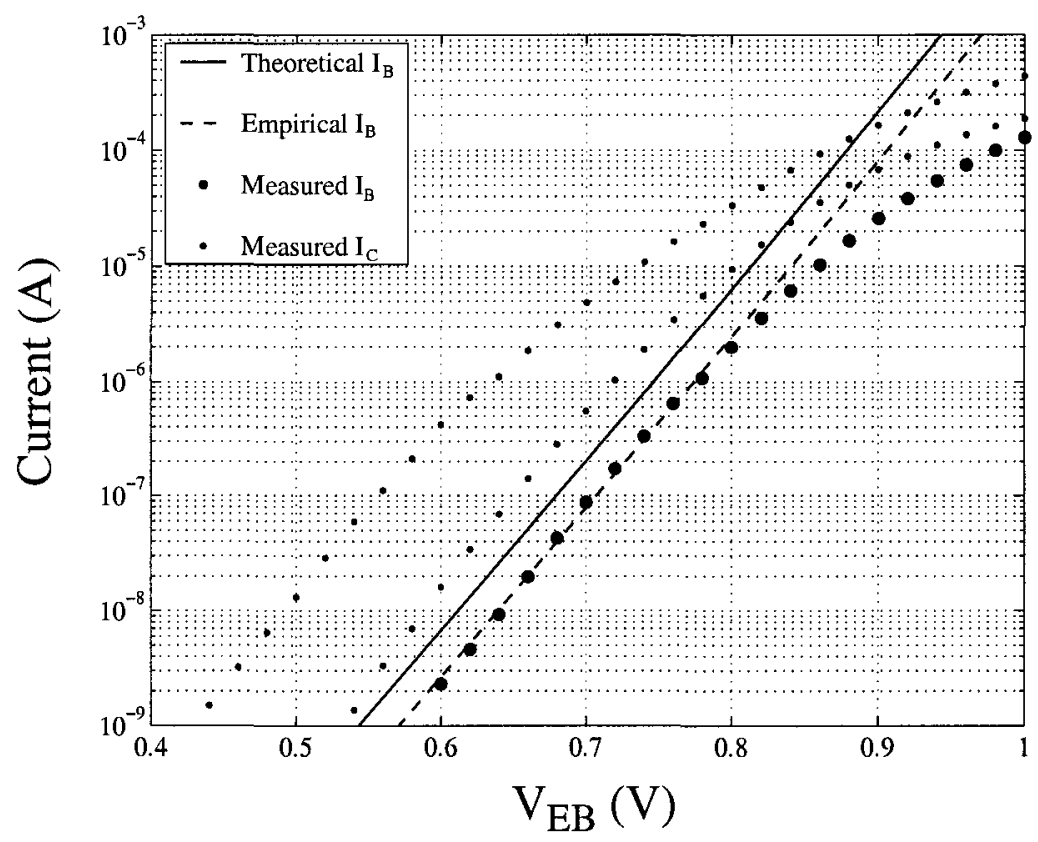

Figure 2.6 Comparison of modelled and measured base and collector currents

\subsection{4 - Early Voltage $\left(V_{A}\right)$}

The Early voltage $\left(V_{A}\right)$ is a fundamental parameter in the operation of a bipolar transistor. It is also a key parameter which has been shown to have a significant impact on the performance of log-domain filters [32]. The Early voltage for a PNP transistor is defined as

$$
V_{A}=\left(\left.\frac{1}{W_{B}} \cdot \frac{d W_{B}}{d V_{C B}}\right|_{V_{\mathrm{CB}=0}}\right)^{-1}
$$

where $W_{B}$ represents the base width, and $V_{C B}$ represents the voltage across the collectorbase junction [22]. A more common expression for Early voltage is given by 


$$
V_{A}=\frac{I_{C}}{r_{o}}
$$

where $r_{o}$ represents the effective output resistance of the transistor. This section will provide a brief derivation of a theoretical value of $V_{A}$, will describe some of the differences observed between the first order theory and the measured results, and will describe the technique used to empirically determine $V_{A}$ from experimental data.

The Early voltage is used to model the variation in the width of the depletion region at the base-collector junction as a function of the base-collector bias voltage. This variation modulates the effective width of the base, giving rise to variation in collector current. An approximation of the Early voltage can be found by relating the derivative in Eqn. (2.14) to the base-collector junction capacitance according to

$$
q N_{B} \cdot \frac{d W_{B}}{d V_{C B}}=c_{B C}
$$

where $c_{B C}$ represents the base-collector junction capacitance per unit area, which can in turn be described as $C_{B C} / A_{B C}$, the entire base-collector junction capacitance divided by the total effective base-collector junction area. The Early voltage can therefore be appoximated as

$$
V_{A}=\frac{q N_{B} W_{B} A_{B C}}{C_{B C}}
$$

as given in [28]. Using the parameters tabulated in the Appendix, the theoretical value of $V_{A}$ would be $2.4 \mathrm{~V}$ for the lateral device and $76 \mathrm{~V}$ for the vertical device. The expected value for the lateral device is extremely (and somewhat unrealistically) low, but demonstrates important relationships, both resulting from the relatively low base doping of these devices. First, since the Early voltage is proportional to $N_{B}$, the value of $V_{A}$ should be expected to be very low when a lightly doped n-well is used to form a base, as is the case here. Second, the Early voltage is proportional to $W_{B}$, and therefore the "Late effect" as described in Section 2.2.2 can be expected to have an impact on the measured value of $V_{A}$. This effect is demonstrated below. 
A comparison of experimentally measured collector currents, taken at two values of collector-base voltage, is shown in Figure 2.7. The dark data points in the figure represent measured currents with $V_{C B}=0 \mathrm{~V}$ at the lateral junction, while the light data points represent the currents with $V_{C B}=-1 \mathrm{~V}$. The lateral base width modulation which results from the increased reverse bias is reflected in the figure by the percentage change in the lateral collector current. The percentage change can be seen to vary over the range of $V_{E B}$, being most pronounced at low values of forward bias (when the base-emitter depletion region penetration is at its maximum). As a result, the measured value of $V_{A}$, which would normally be assumed to be a constant, is in fact an increasing function of $V_{E B}$

It is also worth noting that there is a second and somewhat surprising effect shown by Figure 2.7. The increase in lateral collector current (due to the reverse bias on the lateral junction base collector junction) is accompanied by a decrease in vertical collector current. The two transistor model of Figure 2.2 would assume the complete independence of the two devices, which is shown here not to be an entirely accurate assumption.

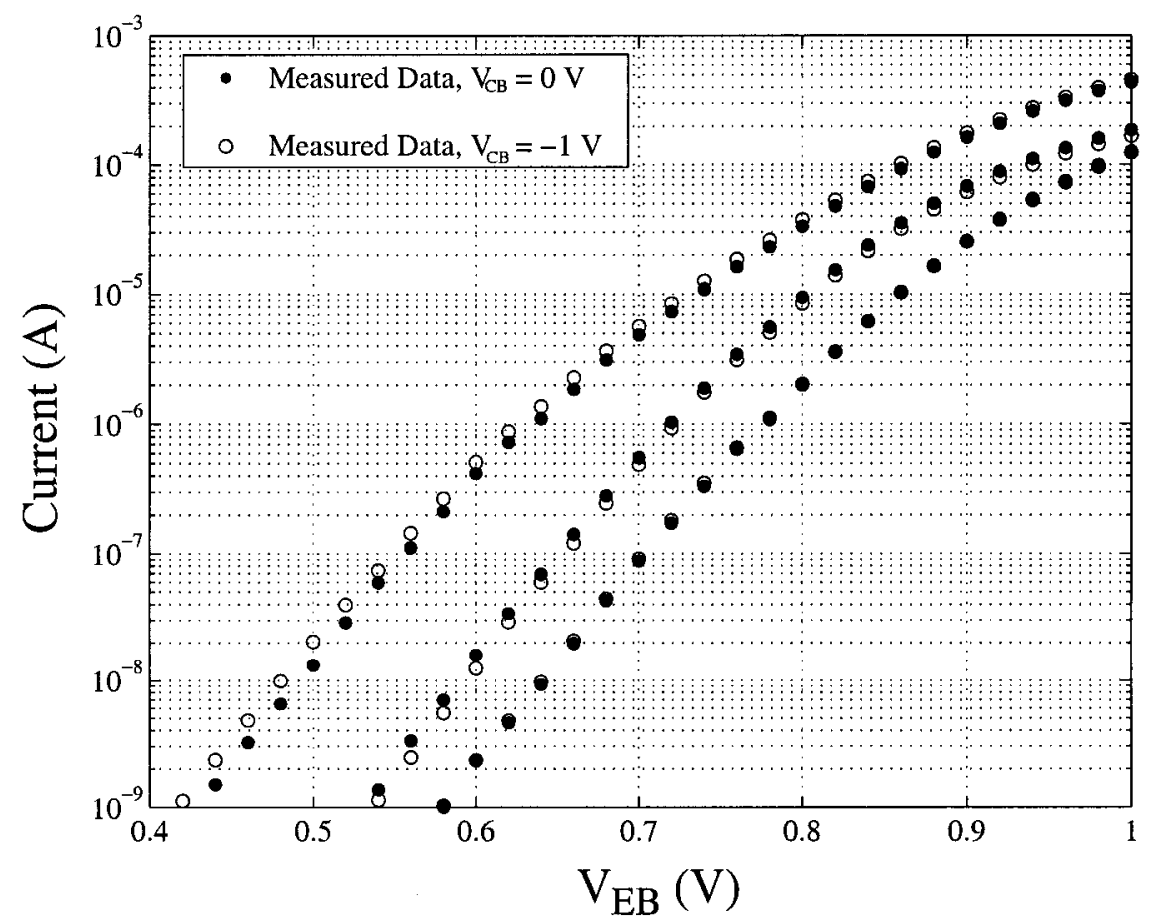

Figure 2.7 Measured base and collector currents with $V_{C B}=0 \mathrm{~V}$ and $V_{C B}=-1 \mathrm{~V}$ 
Neither of these effects can be adequately captured with a simple first order model. In the interests of model simplicity, a constant value of $V_{A}$, measured at the operating point $V_{E B}=0.7 \mathrm{~V}$, has been used. Measured lateral collector currents, plotted as a function of $V_{E C}$ for three values of $V_{E B}$, are shown in Figure 2.8. Each curve once again represents the average measurements taken from three individual devices. Best-fit curves have been included in the figure. The value $V_{A}$ can be extracted from the x-intercept of these best-fit curves. A summary of the extracted values of $V_{A}$ for $V_{E B}=0.6 \mathrm{~V}$ to $V_{E B}=0.8 \mathrm{~V}$ is also provided in the figure. The Early voltage can be seen to be an increasing function of $V_{E B}$ forward bias.

The value of Early voltage for the vertical device can be found in a similar manner shown in Figure 2.7. However, since the vertical collector is formed by the substrate, and since the substrate voltage is normally fixed, the value of $V_{A}$ for the vertical device itself is not a critical parameter to model. It has been measured at $V_{A}=40 \mathrm{~V}$. The two measured values of $V_{A}, 6.8 \mathrm{~V}$ for the lateral device and $40 \mathrm{~V}$ for the vertical device, will be taken for the final device optimization.

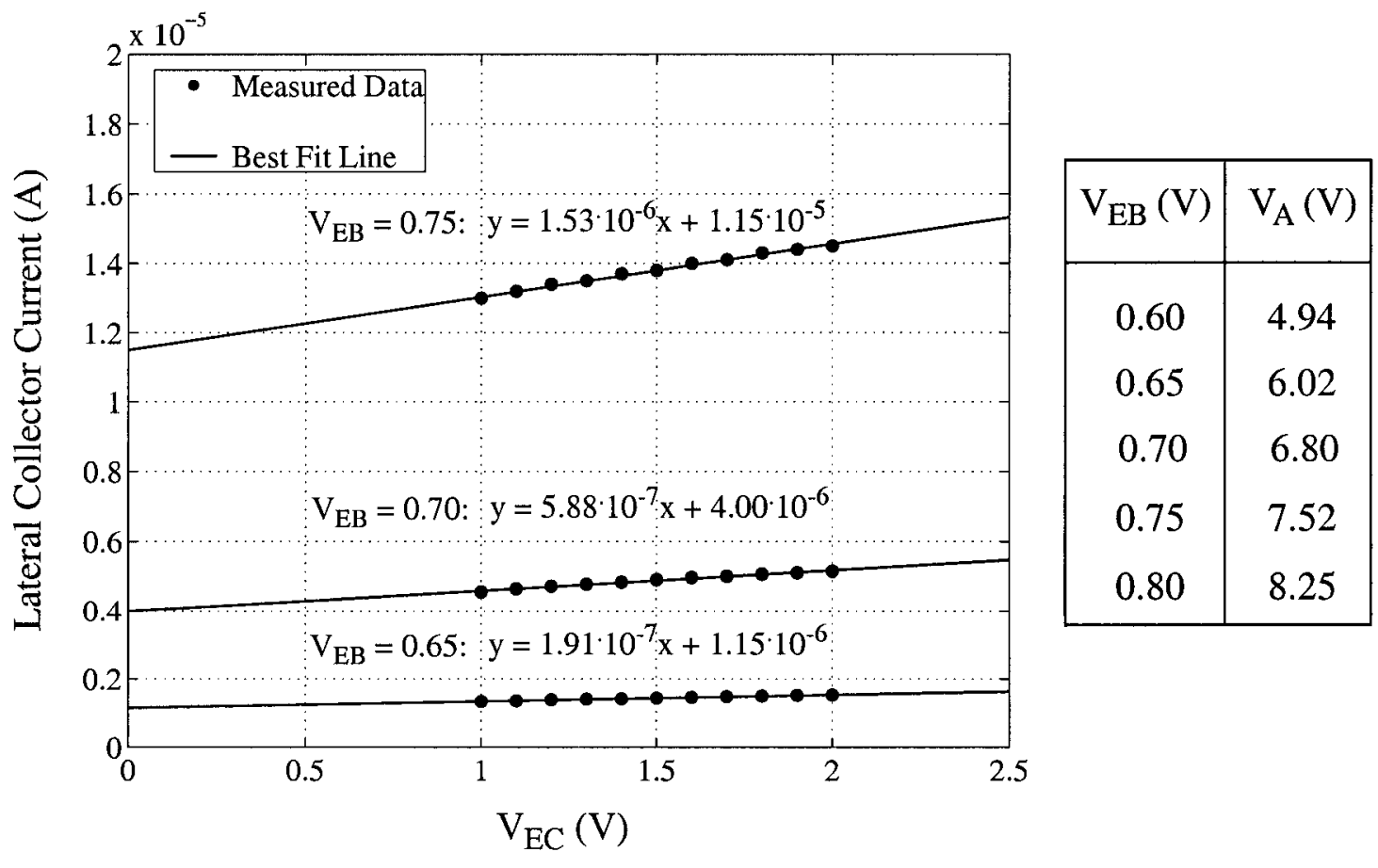

Figure 2.8 Measured collector currents vs $\mathrm{V}_{\mathrm{EC}}$ 


\subsection{5 - Forward Knee Current $\left(I_{K F}\right)$}

The forward knee current $\left(I_{K F}\right)$ is a fundamental parameter used to model highinjection effects in a bipolar transistor. High injection effects, along with the presence of parasitic emitter and base resistances, are largely responsible for the collector current rolloff at large values of $V_{E B}$, as has been noted in the previous sections. This section will provide a brief derivation of a theoretical background to high injection effects and will describe some of the considerations used in determining the value of $I_{K F}$.

The onset of high injection occurs when the minority carrier concentration injected into the base of the device becomes equal to the equilibrium majority carrier concentration in the base [28]. Due to charge neutrality, the majority carrier concentration at the baseemitter junction will be increased by an amount equal to the injected minority carrier concentration. This leads to a non-uniform distribution of majority carriers in the base, which in turn leads to an internal electric field being formed across the base region. The derivation of the expression for collector current, as given in Eqn. (2.2) to Eqn. (2.5), must be modified to include the effect of this electric field.

The previously derived expression for collector current, repeated here for convenience, is given by

$$
I_{C}=\frac{q A_{E B} D_{p} n_{i}^{2}}{N_{B} W_{B}} \cdot e^{\frac{V_{E B}}{v_{T}}}
$$

while the expression for collector current including high injection effects is given by

$$
I_{C}=\frac{2 q A_{E B} D_{p} n_{i}}{W_{B}} \cdot e^{\frac{V_{E B}}{2 v_{T}}}
$$

Note in particular the factor of two in the denominator of the exponent in the second equation. The voltage at which these two expressions for collector current coincide, shown graphically in Figure 2.9, can be found by equating $V_{E B}$ in equations Eqn. (2.18) and Eqn. (2.19), which gives 


$$
V_{K F}=2 v_{T} \ln \left(\frac{2 N_{B}}{n_{i}}\right) .
$$

The corresponding current can be found by substitution of $V_{K F}$ into either collector current expression, which gives

$$
I_{K F}=\frac{4 q A_{E B} D_{p} N_{B} n_{i}}{W_{B}}=I_{s}\left(\frac{2 N_{B}}{n_{i}}\right)^{2}
$$

as given in [28]. $I_{K F}$ is the parameter commonly used to model high injection effects in bipolar transistors. From the parameters tabulated in Appendix, the theoretical value of $I_{K F}$ can be calculated to be $145 \mu \mathrm{A}$ for the lateral device, and $21 \mu \mathrm{A}$ for the vertical device. The corresponding value of $V_{K F}$ is approximately $0.8 \mathrm{~V}$ in each case. The value of each of these parameters is quite low, due largely to the relatively light doping of the base region of these transistors. The fact that theoretical values of $I_{K F}$ and $V_{K F}$ are well within the expected operating regime of these transistors emphasizes the importance of adequately modelling the high injection.

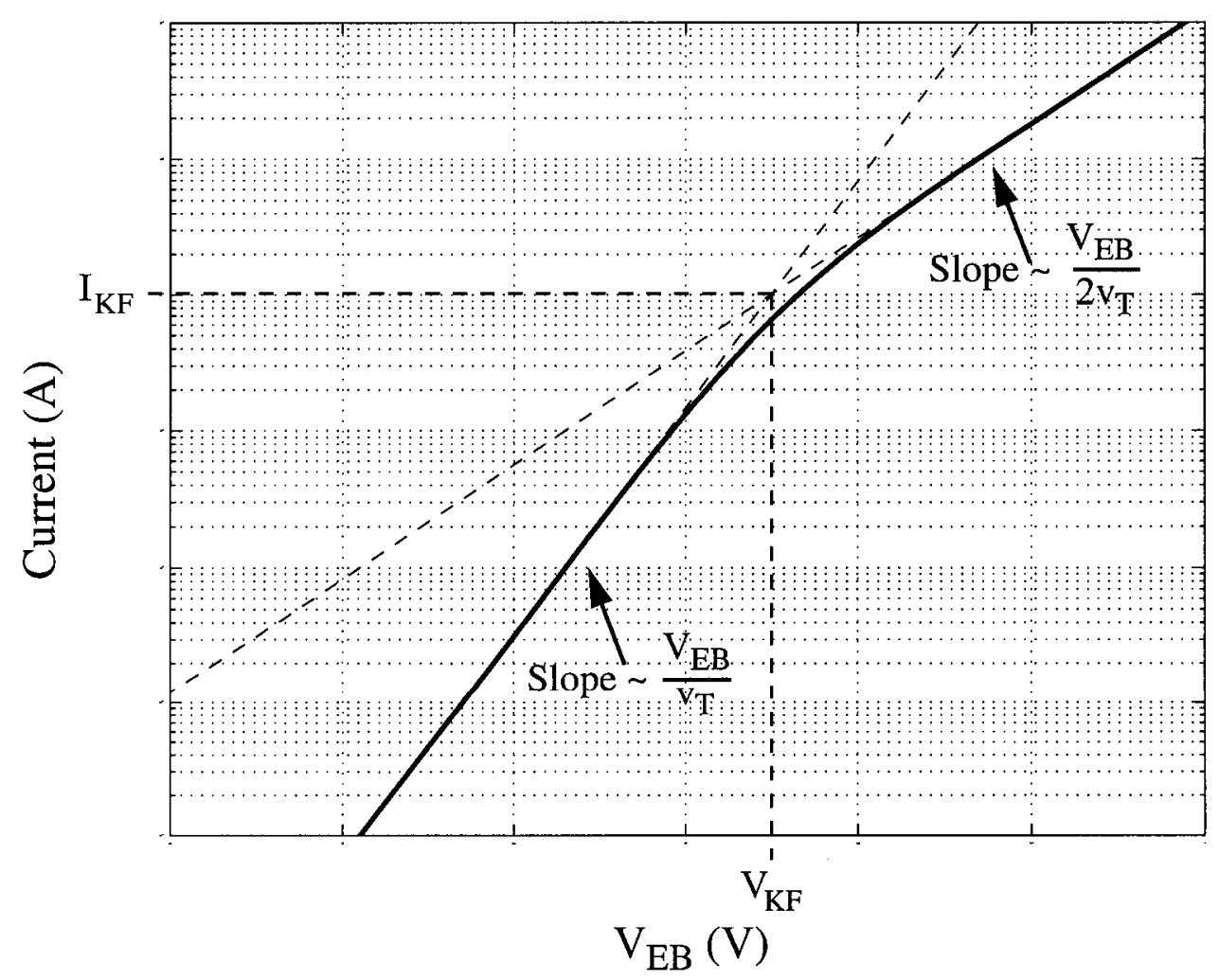

Figure 2.9 Representation of ideal collector current and high-injection effects 
A comparison between the theoretically modelled and experimentally measured values of $I_{K F}$ is difficult to make for several reasons. First, the onset of high injection effects are not abrupt, as shown by the dotted lines in Figure 2.9, but is instead gradual, as shown by the solid line of the figure. Second, the exact location of $I_{K F}$ is obscured by the presence of parasitic emitter and base resistances, which also lead to a reduction of collector currents at large values of forward bias. Third, the Late effect, though minimal at the point at which high injection effects occur, does affect the slope of the collector current in the low-injection, which makes the detection of $I_{K F}$ more difficult. Finally, and perhaps most importantly, since the lateral and vertical devices share a common base and emitter, and are observed to interact with one another, it is not clear from a theoretical standpoint whether the two values of $I_{K F}$ can be effectively measured (or calculated) independently of one another as was assumed above. In view of this, the values of $I_{K F}=145 \mu \mathrm{A}$ for the lateral device and $I_{K F}=21 \mu \mathrm{A}$ for the vertical device were used as the starting values for the final model optimization, but curve fitting in the high-injection region (which also includes the effect of parasitic resistances) must be used to determine the final values of $I_{K F}$.

\subsection{6 - Emitter and Base Resistance $\left(R_{E}\right.$ and $\left.R_{B}\right)$}

The emitter and base resistances, $R_{E}$ and $R_{B}$, are important parasitic parameters that must be taken into account in the operation of a bipolar transistor. These resistances are also important since they have been shown to have a significant impact on the performance of log-domain filters [32]. Since both parameters are parasitic, they are better measured experimentally than predicted theoretically. This section will discuss the techniques used for extracting emitter and base resistances and will provide measured estimates of these resistances.

The most common methods for determining emitter and/or base resistances include DC techniques, high frequency and S-Parameter techniques, pulse-measurement techniques, and noise measurement techniques [22]. Given the nature of log-domain applications, and given the complexity of making high frequency and noise measurements, DC measurement techniques are the most practical in this case. The most 
common DC techniques for determining emitter and base resistance make use of $I_{B}$ vs $V_{E B}$ measurement curves, and several variations on this method are described in [33]. The simplest such method [34] is outlined below.

Typical $I_{B}$ vs $V_{E B}$ characteristics have been shown previously, such as in Figure 2.6. The measured base current deviates from the ideal curve at large values of $V_{E B}$ due primarily to resistive drops across the emitter and base resistances. The voltage generated across these resistances can be expressed as

$$
\Delta V_{E B}=\left[R_{B}+(\beta+1) R_{E}\right] I_{B}
$$

which can be rearranged in the form $y=m x+b$,

$$
\frac{\Delta V_{E B}}{I_{B}}=\beta \cdot R_{E}+\left(R_{E}+R_{B}\right)
$$

where $\beta$ is the independent variable. A best-fit curve, measured at lower values of forward bias, can be used to calculate an ideal value for $V_{E B}$ and $I_{B}$, from which $\Delta V_{E B}$ can be determined. The value of $\frac{\Delta V_{E B}}{I_{B}}$ can then be plotted versus $\beta$, and the values of $R_{E}$ and $R_{B}$ extracted. However, note that this method, as with other DC extraction techniques, is made more complicated by the unique two device structure of these transistors. Because the base and emitters of each device are connected through common terminals, the value of $I_{B}$, and therefore $\beta$, cannot be individually determined. To most accurately estimate the values of $R_{E}$ and $R_{B}$ using this method, three different sets of data have been analysed.

For the first two sets of data, an assumption has been made that the total base current can be divided into two equal contributions, attributed to the lateral and to the vertical device. A value of $\beta$ can therefore be calculated in each case, and Eqn. (2.23) plotted for each device. For a third set of data, the assumption has been made that the two devices can be treated as a single unit. The value of $\beta$ can therefore be calculated based on the combined base and collector currents, and Eqn. (2.23) plotted once again. All three plots, along with best-fit curves, are shown in Figure 2.10. 


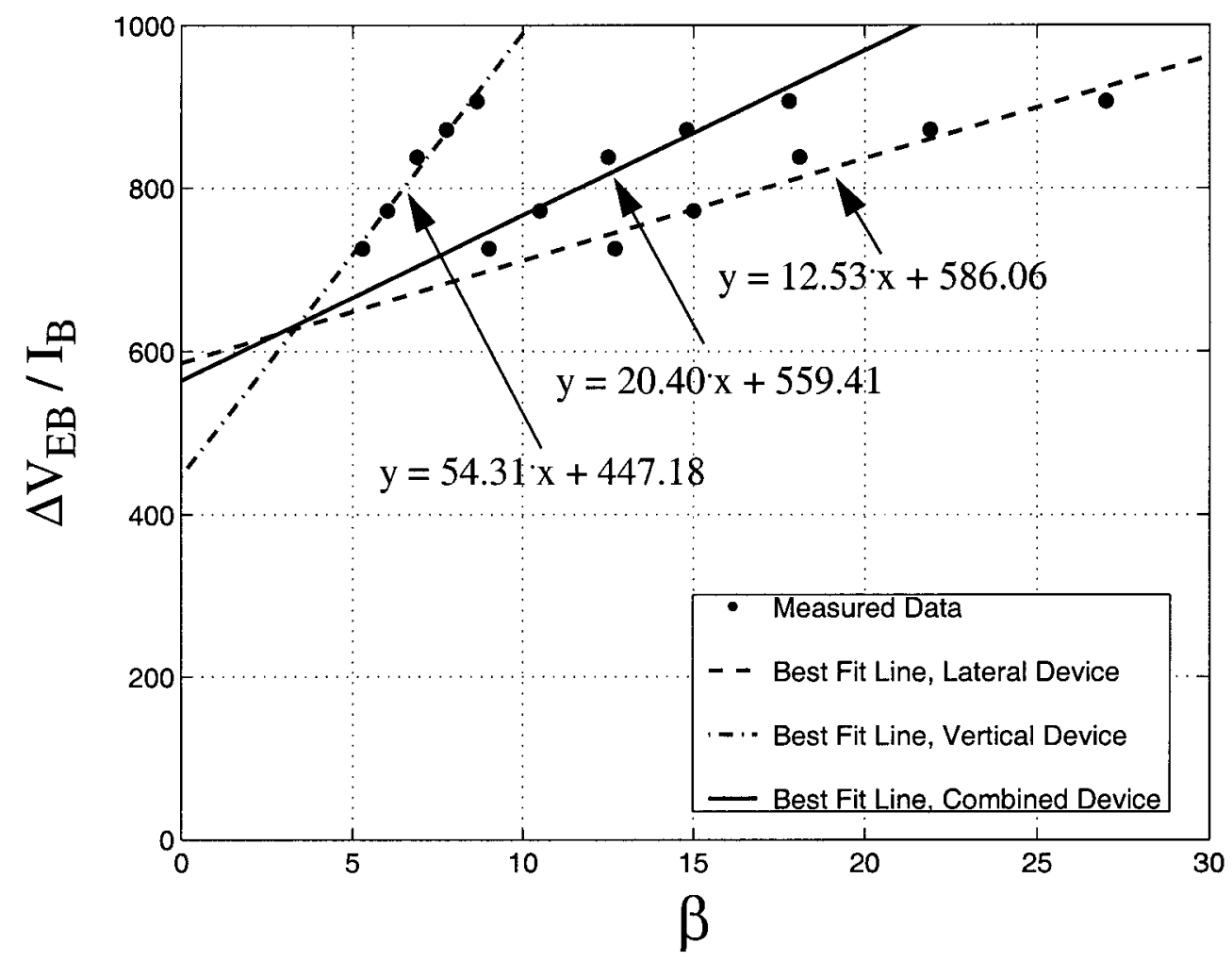

Figure 2.10 Best-fit curve for measurement of $R_{E}$ and $R_{B}$

The data in Figure 2.10 has been taken from $V_{E B}=0.8 \mathrm{~V}$ to $V_{E B}=0.9 \mathrm{~V}$, the minimum range of values for which a significant value of $\Delta V_{E B}$ could be measured. All values of $I_{B}$ and $I_{C}$ have been based on measurements from three individual devices.

From Figure 2.10, the extracted value of $R_{E}$ varies from $12.5 \Omega$ to $54.3 \Omega$, with an average of $29 \Omega$. The value of $R_{B}$ varies from $393 \Omega$ to $574 \Omega$, with an average of close to $500 \Omega$. The slope of the curves for the lateral and vertical devices was found to be very sensitive to the proportion of base current attributed to each device (chosen arbitrarily to be equal between the two devices) which partially explains the wide variation observed in the value for $R_{E}$.

To provide a verification of the value of emitter resistance, the open collector method [22] was used. Using this technique, the collector of the transistor is left in an open circuit condition, and the voltage at the collector can be expressed as 


$$
V_{E C}=v_{T} \ln \left(\frac{1}{\alpha_{R}}\right)+I_{B} R_{E}
$$

where $\alpha_{\mathrm{R}}$ represents the reverse current transfer ratio $\left(\alpha_{R}=\frac{\beta_{R}}{\beta_{R}+1}\right)$ and $I_{B}=I_{E}$. A plot of $I_{B}$ vs $V_{E C}$ produces a characteristic curve such as the one shown in Figure 2.11. The inverse of the slope of the curve in its linear region provides a measurement of $R_{E}$. The data in this case has been taken with both lateral and vertical collectors open, and once again taken from three individual devices.

The value of $R_{E}$ has been measured to be between $35 \Omega-45 \Omega$ in reasonable agreement with the average measured value of $R_{E}$ extrapolated from Eqn. (2.23). The values of $R_{E}=35 \Omega$ and $R_{B}=500 \Omega$, will be used as the starting point in the model optimization for both the lateral and vertical devices, and curve fitting (which will include the effect of $I_{K F}$ ) will be required to determine the final values of the emitter and base resistances.

Note that the collector resistance, $R_{C}$, has not been included within the SPICE model for the sake of simplicity. Based on geometric considerations, the value of $R_{C}$ can be assumed to be lower than $R_{E}$, and the inclusion of a collector resistance of this size does not have any significant impact on the simulated log-domain circuit performance.

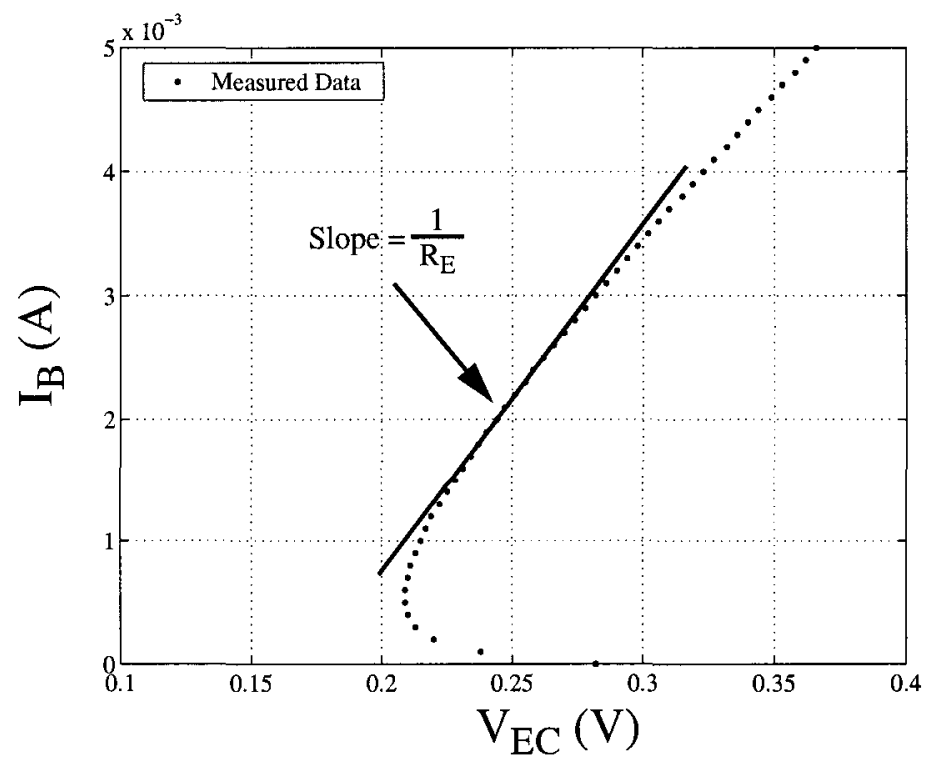

Figure 2.11 Plot of $I_{B}$ vs $V_{E C}$ used in measuring $R_{E}$ 


\subsection{7 - Forward Base Transit Time $\left(\tau_{F}\right)$}

The forward base transit time $\left(\tau_{F}\right)$ is one of the most important parameters in defining the frequency response of a transistor. Base transit time is defined according to

$$
\tau_{F}=\frac{Q_{b}}{I_{C}}
$$

where $Q_{b}$ represents the total dynamic minority carrier charge in the base, and $I_{C}$ represents collector current, leading to the physical interpretation that $\tau_{F}$ represents the average time required for minority carriers to diffuse from the emitter to collector of the device. The parameter $\tau_{F}$ is used to define the total base charging capacitance of the transistor, $C_{b}$, one of three important capacitances which primarily determine the transistor frequency response. The value of $C_{b}$ can be expressed as

$$
C_{b}=\tau_{F} g_{m}
$$

where $g_{m}$ represents the transconductance of the device. The value of $\tau_{F}$ can also be used to determine the upper bound on the maximum theoretically operating frequency of the device, which can be defined as

$$
f_{\text {Tmaximum }}=\frac{1}{2 \pi \tau_{F}}
$$

where $f_{\text {Tmaximum }}$ represents the maximum obtainable unity gain frequency of the transistor.

The value of $Q_{b}$ is a function of the emitter-base forward bias voltage. Assuming a simple rectangular base geometry and a charge distribution which varies linearly from $\mathrm{p}_{\mathrm{n}(\mathrm{ebj})}$ at the emitter to zero at the collector, the value of $Q_{b}$ can be expressed as

$$
Q_{b}=\frac{1}{2} q p_{n(e b j)} A_{E B} W_{B}=\frac{1}{2} q A_{E B} W_{B} \cdot \frac{n_{i}^{2}}{N_{B}} e^{\frac{V_{E B}}{v_{T}}}
$$


Combining equations (2.5), (2.25), and (2.28) provides the following simple expression often used to calculate the base transit time [27]-[26],

$$
\tau_{F}=\frac{W_{B}^{2}}{2 D_{p}}
$$

However, when working with two separate collectors (and working within a circular structure) the derivation of $Q_{b}$ is more complex. This section will present an analysis adapted from [29] that will be used to estimate the value of $Q_{b}$ which can be attributed to both the lateral and vertical devices. From these values, the base transit time, $\tau_{F}$, can be determined. Note that an experimental verification of the theoretical values of $\tau_{F}$ has not been performed in this case.

A cross sectional diagram, which can be used to approximate the minority charge distribution within a lateral PNP transistor, is shown in Figure 2.12. The three-dimensional cylindrical region below the emitter can be defined as $Q_{b 1}$, and the charge distribution within this cylinder can be modelled as varying uniformly in the vertical direction, from $p_{n(e b j)}$ at the emitter junction to zero at the substrate junction. The hollow cylindrical shell between the emitter and lateral collector can be defined as $Q_{b 2}$, and the charge distribution in this case can be modelled as varying uniformly in the radial direction, from $p_{n(e b j)}$ at the emitter to zero at the lateral collector. The remaining cylindrical shell can be defined as $Q_{b 3}$, and the charge

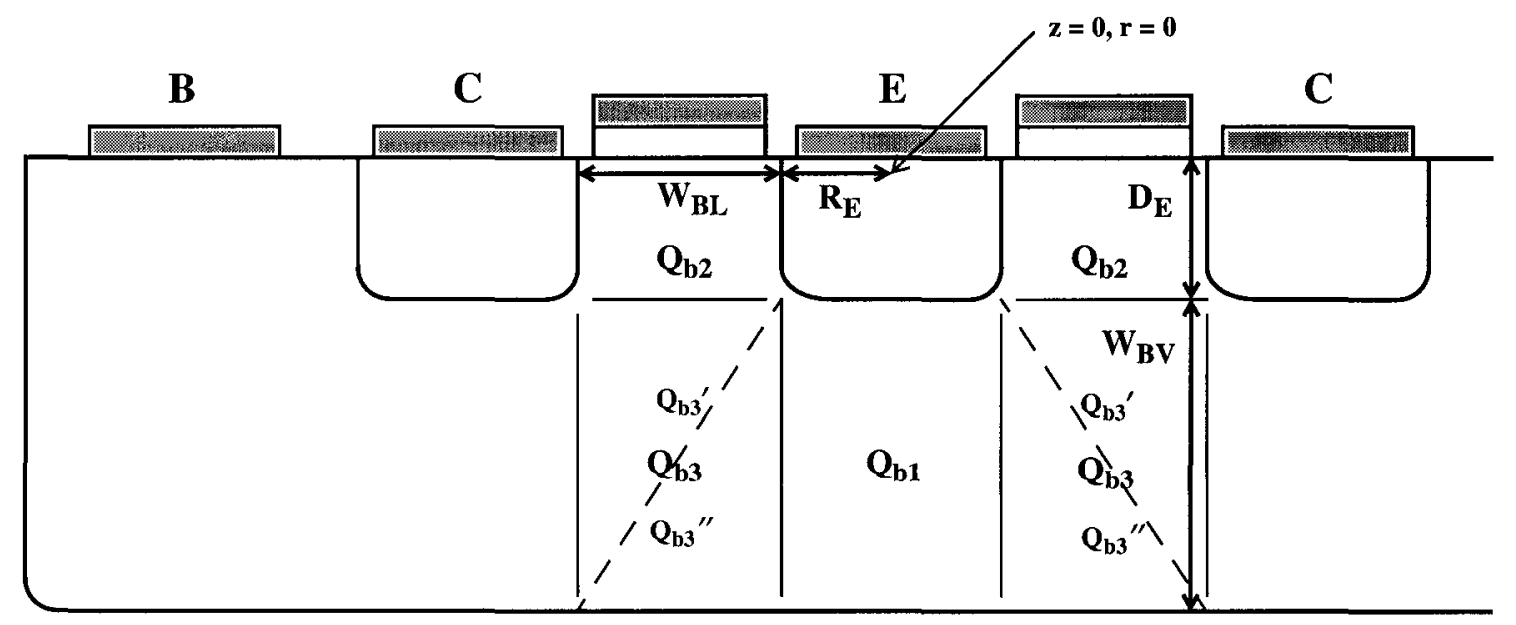

SUB

Figure 2.12 Cross sectional diagram of a lateral PNP minority charge distribution model (vertical axis not to scale) 
distribution can be modelled as varying in both the vertical and radial directions, from $p_{n(e b j)}$ at the emitter edge to zero at both the substrate junction and zero at cylinder's exterior edges. The minority charge outside these cylindrical shells can be treated as negligible [29] and will be omitted for the purposes of this analysis.

The total charge in the $Q_{b I}$ region can be found by integration along the vertical dimension of the vertical base region. Using the dimensions as defined Figure 2.12, the value of $Q_{b 1}$ can be approximated as

$$
\begin{gathered}
Q_{b 1}=q p_{n(e b j)} \int_{\mathrm{z}=\mathrm{D}_{E}}^{z=\mathrm{D}_{E}+\mathrm{W}_{B V}} \int_{\mathrm{r}=0}^{\mathrm{r}=\mathrm{R}_{E}}\left(1-\frac{z-D_{E}}{W_{B V}}\right) \cdot 2 \pi r d r d z \\
=q p_{n(e b j)} \cdot \frac{\pi}{2} \cdot W_{B V} R_{E}^{2} .
\end{gathered}
$$

Similarly, the total charge in the $Q_{b 2}$ region can be approximated as

$$
\begin{aligned}
Q_{b 2} & =q p_{n(e b j)} \int_{z=0}^{z=\mathrm{D}_{E}} \int_{\mathrm{r}=\mathrm{R}_{E}}^{\mathrm{r}=\mathrm{R}_{E}+W_{B L}}\left(1-\frac{r-R_{E}}{W_{B L}}\right) \cdot 2 \pi r d r d z \\
& =q p_{n(e b j)} \cdot \pi \cdot W_{B L} D_{E}\left(R_{E}+\frac{W_{B L}}{3}\right)
\end{aligned}
$$

and the total charge in the $Q_{b 3}$ region can be approximated as

$$
\begin{gathered}
Q_{b 3}=q p_{n(e b j)} \int_{\mathrm{z}=\mathrm{D}_{E}}^{z=\mathrm{D}_{E}+\mathrm{W}_{B V}} \int_{\mathrm{r}=\mathrm{R}_{E}}^{\mathrm{r}=\mathrm{R}_{E}+W_{B L}}\left(1-\frac{z-D_{E}}{W_{B V}}\right)\left(1-\frac{r-R_{E}}{W_{B L}}\right) \cdot 2 \pi r d r d z \\
=q p_{n(e b j)} \cdot \pi \cdot W_{B L} W_{B V}\left(\frac{R_{E}}{2}+\frac{W_{B L}}{6}\right) .
\end{gathered}
$$

The total dynamic minority charge in the base can therefore be calculated from the sum of $Q_{b 1}, Q_{b 2}$, and $Q_{b 3}$, which depend only on $p_{n(e b j)}$ and geometrical parameters.

The question remains as to the quantity of the charge which should be attributed to the lateral and vertical components of the transistor. As shown in Figure 2.12, a line has been drawn between two corners of the $Q_{b 3}$ region. It has been assumed that charge which lies above this partition, including $Q_{b 2}$ and the region labelled as $Q_{b 3}{ }^{\prime}$, can be considered to belong to the lateral device, and that charge which lies below this partition, including $Q_{b 1}$ and 
the region labelled as $Q_{b 3}{ }^{\prime \prime}$, can be considered to belong the vertical device. The values of $Q_{b 3}{ }^{\prime}$ and $Q_{b 3}{ }^{\prime \prime}$ can be calculated by modifying the limits of integration in Eqn. (2.32), and are given by

$$
\begin{aligned}
& Q_{b 3}{ }^{\prime}=q p_{n(e b j)} \int_{\mathrm{z}=\mathrm{D}_{E}}^{z=\mathrm{D}_{E}+\mathrm{W}_{B V}} \int_{r=R_{E}+\left(\frac{z-D_{E}}{W_{B V}}\right) W_{B L}}^{r=R_{E}+W_{B L}}\left(1-\frac{z-D_{E}}{W_{B V}}\right)\left(1-\frac{r-R_{E}}{W_{B L}}\right) \cdot 2 \pi r d r d z \\
& =q p_{n(e b j)} \cdot \pi \cdot W_{B L} W_{B V}\left(\frac{R_{E}}{4}+\frac{7 \cdot W_{B L}}{60}\right) \\
& z=\mathrm{D}_{E}+\mathrm{W}_{B V} \quad r=R_{E}+\left(\frac{z-D_{E}}{W_{B V}}\right) W_{B L} \\
& Q_{b 3}{ }^{\prime \prime}=q p_{n(e b j)} \int_{\mathrm{z}=\mathrm{D}_{E}}^{{ }} \int_{\mathrm{r}=\mathrm{R}_{E}}\left(1-\frac{z-D_{E}}{W_{B V}}\right)\left(1-\frac{r-R_{E}}{W_{B L}}\right) \cdot 2 \pi r d r d z \\
& =q p_{n(e b j)} \cdot \pi \cdot W_{B L} W_{B V}\left(\frac{R_{E}}{4}+\frac{W_{B L}}{20}\right)
\end{aligned}
$$

The base transit times can then be calculated using equations (2.5) and (2.30) - (2.34), according to the expressions

$$
\tau_{F(\text { lateral })} \approx \frac{Q_{b 2}+Q_{b 3}^{\prime}}{I_{c(\text { lateral })}}, \quad \tau_{F(\text { vertical })} \approx \frac{Q_{b 1}+Q_{b 3}{ }^{\prime \prime}}{I_{c(\text { vertical })}}
$$

Using the dimensions given in the Appendix, the values of base transit time can be estimated as $100 \mathrm{ps}$ for the lateral device and $1.3 \mathrm{~ns}$ for the vertical device, and these values have been used as the SPICE parameters for the final transistor model.

Note that there are many approximations and assumptions made in the analysis above. In particular, note that the minority charge under the lateral collector to the base contact has not been included in the analysis, and therefore the estimate of $\tau_{\mathrm{F}}$ is almost certainly an underestimate. Also note that this sort of derivation makes the assumption that the base is uniformly doped, which is likely not the case for this transistor. Despite these drawbacks, the high-frequency modelling of the transistor was found to be adequate for the circuit designed in this work, as will be discussed in detail in Chapters 3 and 4 . 


\subsection{8 - Parasitic Capacitance Parameters}

The final set of parameters which must be discussed are those associated with parasitic capacitances. Since information about the parasitic capacitances is provided by the manufacturer, these model parameters have been adapted from the manufacturer's literature and/or extracted using the available software layout tools. This section will briefly describe the parasitic parameters of interest, and provide a tabulation of the parameters to be used in the transistor model.

The two most important capacitances used to model the frequency response of a bipolar transistor are $C_{\pi}$ and $C_{\mu}$. The value of $C_{\pi}$ is defined as the sum of the base charging capacitance $C_{b}$, defined previously by Eqn. (2.26), and the parasitic emitter-base junction capacitance $C_{j e}$. The value of $C_{\mu}$ is defined as the parasitic collector-base junction capacitance $C_{j c}$. These parasitic capacitances are given by the two expressions

$$
\begin{gathered}
C_{j e}=\frac{C_{j e o}}{\left(1-\frac{V_{E B}}{V_{j e}}\right)^{m_{j e}}}, \\
C_{j c}=\frac{C_{j c o}}{\left(1-\frac{V_{C B}}{V_{j c}}\right)^{m_{j c}}}
\end{gathered}
$$

where $C_{j e o}$ and $C_{j c o}$ represent the zero bias capacitance across the emitter-base and collector base junctions respectively, $V_{E B}$ and $V_{C B}$ represent the built-in potentials of respective junctions, and $m_{j e}$ and $m_{j c}$ represent the grading coefficients of each respective junction. The effect of these parasitic capacitances on the transition frequency of the transistor, $f_{T}$, is given in [27] and can be expressed as

$$
f_{T}=\frac{1}{2 \pi} \cdot \frac{g_{m}}{C_{\pi}+C_{\mu}}=\frac{1}{2 \pi} \cdot \frac{1}{\left(\tau_{F}+\frac{C_{j e}}{g_{m}}+\frac{C_{j c}}{g_{m}}\right)} .
$$

One additional capacitance which must be included in this model is the gate to base capacitance, $C_{g b}$, formed by the MOSFET transistor, as shown by $M_{l}$ in 
Figure 2.1(b) and shown schematically in Figure 2.2(a). This capacitance does not fit in any standard bipolar SPICE model, and will be included as a stand-alone capacitance. The values of each of the parasitic capacitance parameters described above are provided below in Table 2.2.

Table 2.2:Parasitic Capacitance Parameters for the SPICE model

\begin{tabular}{|c|c|c|}
\hline Model Parameter & Lateral Device & Vertical Device \\
\hline \hline $\mathrm{C}_{\mathrm{jeo}}$ & $3.6 \mathrm{fF}$ & $3.6 \mathrm{fF}$ \\
\hline $\mathrm{V}_{\mathrm{je}}$ & $0.9 \mathrm{~V}$ & $0.9 \mathrm{~V}$ \\
\hline $\mathrm{M}_{\mathrm{je}}$ & 0.55 & 0.55 \\
\hline $\mathrm{C}_{\mathrm{jco}}$ & $25 \mathrm{fF}$ & $10 \mathrm{fF}$ \\
\hline $\mathrm{V}_{\mathrm{jc}}$ & $0.9 \mathrm{~V}$ & $0.55 \mathrm{~V}$ \\
\hline $\mathrm{M}_{\mathrm{jc}}$ & 0.55 & 0.35 \\
\hline \multicolumn{3}{|c|}{ Gate-Base Capacitance: $12 \mathrm{fF}$} \\
\hline
\end{tabular}

\section{3 - Lateral PNP Transistors: Complete SPICE Model}

A complete SPICE model of the lateral PNP transistor has been developed. The parameters described in Section 2.2 have been used for this model, and it will be shown that this set of parameters is sufficient to adequately capture the behaviour of the transistor. This section will present the initial SPICE model, briefly describe the model optimization, and present the final and complete SPICE model for the lateral PNP transistor.

An initial SPICE model has been developed based on the parameter values calculated and measured in Section 2.2, and are summarized in Table 2.3. A comparison of the DC characteristics of the modelled and the experimentally measured response is shown in Figure 2.13. Due to the interaction between the parameters in the combined model, the predicted DC characteristics shown in the figure are not ideal. In particular, there are significant deviations in the model for large values of $V_{E B}$ forward bias, 
indicating that high injection effects and parasitic base and emitter resistances have not been modelled accurately.

An optimization of the model has been performed. It was found that the values of $I_{K F}$ for the lateral device did not sufficiently model the roll-off of the lateral collector current. By reducing the value of $I_{K F}$ to that of the vertical device, a much better fit was generated. Since both devices have been observed to interact closely with one another, using a single value of $I_{K F}$ for both devices is reasonable. The final optimized value was $30 \mu \mathrm{A}$ for both devices.

It was also found that values of $R_{E}$ and $R_{B}$ were not sufficiently large to model the roll-off of the base current. Since direct open collector measurements had indicated the value of $R_{E}$ to be close to $35 \Omega$, already a large value for emitter resistance, it was the value of base resistance which was increased. A value of $R_{B}$ equal to $1000 \Omega$ provided the best results.

To further improve the fit, the value of $\beta$ was decreased to 120 for the lateral device and 15 for the vertical device, closer to the theoretically expected values. To more accurately capture the overall slopes of the collector currents, the values of $n_{F}$ were increased to 1.25 for the lateral device and 1.10 for the vertical device, and the values of $I_{S}$ fit accordingly. The parameters $V_{A}, \tau_{F}$, and the parasitic capacitances were not adjusted in the optimization.

The optimized parameter values for the final transistor model are summarized in Table 2.4. A comparison of the DC characteristics of the modelled response and the experimentally measured response is shown in Figure 2.14. Note in particular the fit at $V_{E B}=0.7 \mathrm{~V}$, the point about which the optimization has been performed. A linear plot of the modelled and experimentally measured responses has also been included in Figure 2.15 in order to more clearly show the high-bias current agreement. 
Table 2.3:Initial values for SPICE lateral PNP Model

\begin{tabular}{|c|c|c|c|c|c|}
\hline $\begin{array}{c}\text { SPICE } \\
\text { Parameter }\end{array}$ & Lateral & Vertical & $\begin{array}{c}\text { SPICE } \\
\text { Parameter }\end{array}$ & Lateral & Vertical \\
\hline \hline IS & $1.2 \times 10^{-15}$ & $2.7 \times 10^{-18}$ & TF & $1.0 \times 10^{-10}$ & $1.3 \times 10^{-9}$ \\
\hline NF & 1.19 & 1.03 & CJE & $3.6 \times 10^{-15}$ & $3.6 \times 10^{-15}$ \\
\hline BETA & 150 & 20 & VJE & 0.9 & 0.9 \\
\hline VAF & 6.5 & 40 & MJE & 0.55 & 0.55 \\
\hline IKF & $1.45 \times 10^{-4}$ & $2.1 \times 10^{-5}$ & CJC & $2.5 \times 10^{-14}$ & $1.0 \times 10^{-14}$ \\
\hline RE & 35 & 35 & VJC & 0.9 & 0.55 \\
\hline RB & 500 & 500 & MJC & 0.55 & 0.35 \\
\hline \multicolumn{7}{|c|}{ Gate Base Capacitance: $1.2 \times 10^{-14}$} \\
\hline
\end{tabular}

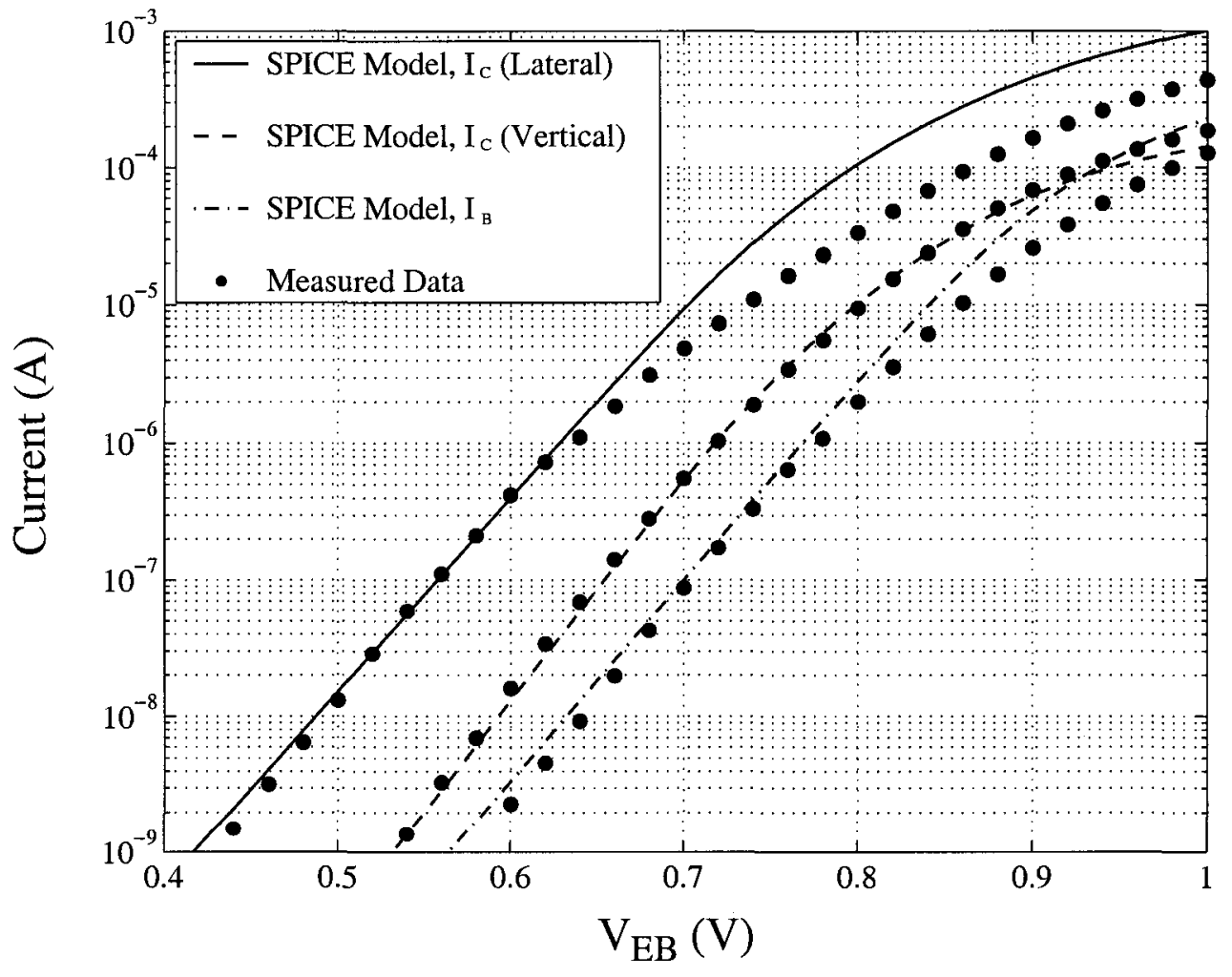

Figure 2.13 Initial DC characteristic curves for lateral PNP SPICE model 
Table 2.4:Optimized values for SPICE lateral PNP Model

\begin{tabular}{|c|c|c|c|c|c|}
\hline $\begin{array}{c}\text { SPICE } \\
\text { Parameter }\end{array}$ & Lateral & Vertical & $\begin{array}{c}\text { SPICE } \\
\text { Parameter }\end{array}$ & Lateral & Vertical \\
\hline \hline IS & $1.8 \times 10^{-15}$ & $1.0 \times 10^{-17}$ & TF & $1.0 \times 10^{-10}$ & $1.3 \times 10^{-9}$ \\
\hline NF & 1.25 & 1.10 & CJE & $3.6 \times 10^{-15}$ & $3.6 \times 10^{-15}$ \\
\hline BETA & 120 & 15 & VJE & 0.9 & 0.9 \\
\hline VAF & 6.5 & 40 & MJE & 0.55 & 0.55 \\
\hline IKF & $3.0 \times 10^{-5}$ & $3.0 \times 10^{-5}$ & CJC & $2.5 \times 10^{-14}$ & $1.0 \times 10^{-14}$ \\
\hline RE & 35 & 35 & VJC & 0.9 & 0.55 \\
\hline RB & 1000 & 1000 & MJC & 0.55 & 0.35 \\
\hline \multicolumn{7}{|c}{ Gate Base Capacitance: $1.2 \times 10^{-14}$} \\
\hline
\end{tabular}

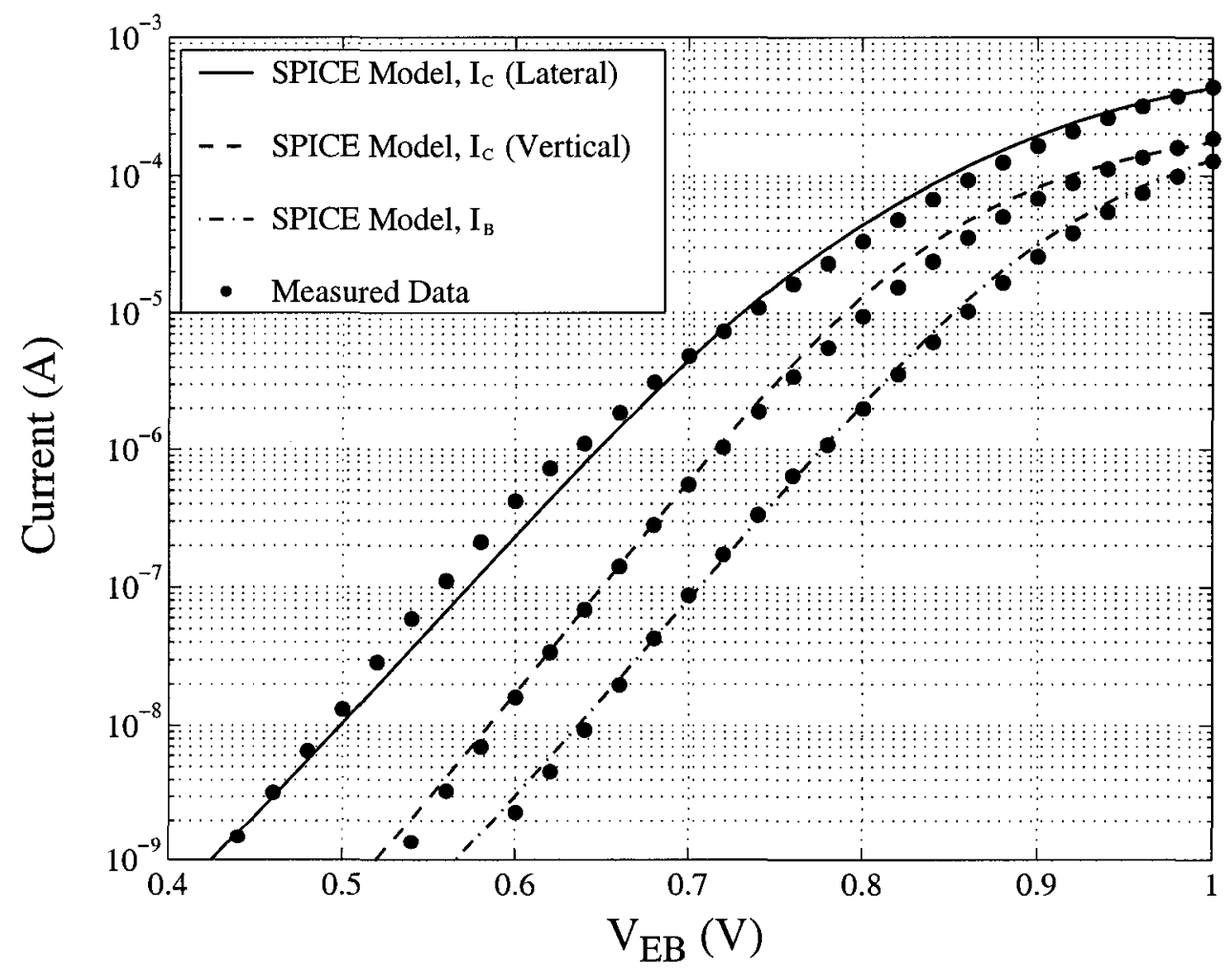

Figure 2.14 Optimized DC characteristic curves for lateral PNP SPICE model 


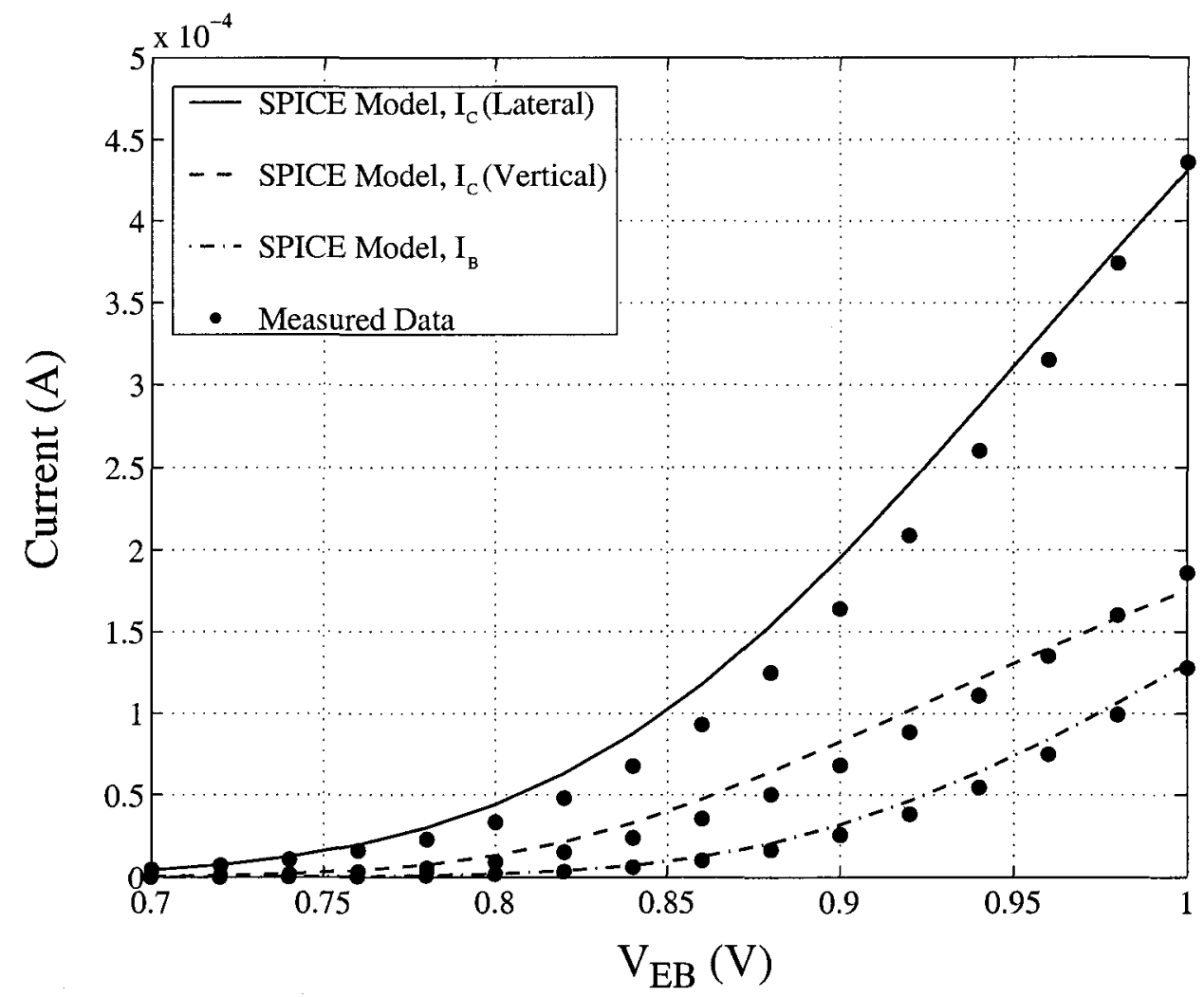

Figure 2.15 Linear plot of DC characteristic curves for lateral PNP SPICE model

Since the high frequency performance of these devices was not directly measured, no optimization or comparison of the high frequency performance was undertaken. Furthermore, the evaluation of parameters such as the transition frequency, $f_{T}$, are somewhat difficult to define, since the base current of the lateral and vertical transistors are connected through a common terminal. However, if the effective current gain is defined as

$$
\beta_{e f f}=I_{C(\text { lateral })} / I_{B(\text { lateral }+ \text { vertical })}
$$

then a number similar in nature to the transition frequency $f_{T}$ can be calculated. In simulation, the value of this modified value of $\mathrm{f}_{\mathrm{T}}$ was found to be $320 \mathrm{MHz}$ at $10 \mu \mathrm{A}$, and $490 \mathrm{MHz}$ at $100 \mu \mathrm{A}$. While these values would appear to overestimate of the performance of these lateral PNP devices, it will be demonstrated in Chapter 3 and 4 that log-domain filter circuits which make use these devices can operate at frequencies at over $10 \mathrm{MHz}$. It 
has previously been shown that log-domain filters can be made to operate up to frequencies of approximately $1 / 10$ of the $f_{T}$ of the slowest transistors [5]. If this were the case in this work as well, it would suggest that the effective $f_{T}$ of the lateral PNP transistors would be on the order of $100 \mathrm{MHz}$ or higher, well within a factor of ten of the effective $f_{T}$ values above from simulation. Therefore, while the lateral PNP model can be expected to overestimate frequency performance (due in large part to an underestimation of $\tau_{\mathrm{F}}$ ), the modelled transistor frequency response is still reasonable, based on experimental measurements from working log-domain circuits.

In conclusion, a SPICE compatible model has been developed for a lateral PNP transistor fabricated in $0.35 \mu \mathrm{CMOS}$ technology. The model requires only fifteen parameters in total, each of which can be estimated based on physical considerations, extracted from straightforward device measurements, or adopted from standard information provided by the manufacturer. The model is sufficiently simple that it can be readily developed in any CMOS technology.

This model will form the basis for all the designs and simulations of the logdomain filter circuits described in the subsequent chapters, and will be used to demonstrate the feasibility of implementing circuits using the lateral PNP transistor in CMOS technology. 


\section{Chapter 3 - The Design of a Log-Domain}

\section{Integrator in CMOS}

\section{Technology}

In the previous chapter, it has been shown that lateral PNP transistors with promising characteristics can be fabricated in CMOS technology. These transistors can be used to implement a log-domain integrator, a circuit which serves as a fundamental building block for log-domain filter designs. In this chapter, a log-domain integrator, developed in $0.35 \mu$ CMOS technology, will be presented.

Several important designs for log-domain integrators have been described in the literature [1]. Of these, some can be implemented using only NPN transistors in the signal path. Such implementations are of particular interest, since these circuits can be mapped directly in CMOS technology using the lateral PNP transistor. However, as is the case whenever a circuit design is mapped into a new technology, there are several considerations which must be taken into account. This chapter will describe the process of developing the log-domain integrator in CMOS technology.

The outline of this chapter is as follows: the first section will provide the theoretical background required to describe the operation of a log-domain integrator. The second section will describe the methodology of mapping the log-domain integrator into 
CMOS technology, as well as describing the required interface circuitry. The final section will present both simulated results and experimental measurements of the log-domain integrator, demonstrating both the capabilities and limitations of this new log-domain filtering approach.

\section{1 - Fundamentals of Log-Domain Integrator Design}

The following section will provide the background required to describe the firstorder operation of a log-domain integrator. A method of mathematical notation will be introduced and applied to a simple, all-bipolar log-domain integrator circuit. In addition, an important variation of the circuit, the damped log-domain integrator, will be described. The damped integrator circuit will be used for simulations and experimental measurements of the integrator performance provided later in this chapter.

\subsection{1 - The Log-Domain Cell and the LOG(x) and EXP(x) Operators}

A basic log-domain cell is shown below in Figure 3.1. Only two PNP transistors are required for the cell, yet it forms the heart of all the log-domain circuitry to be presented in this chapter. To derive the transfer function for this cell for the general case, the circuit can be treated as a translinear loop. The sum of the voltages around this loop can be expressed as

$$
V_{1}-V_{E B 1}+V_{E B 2}-V_{2}=0 .
$$

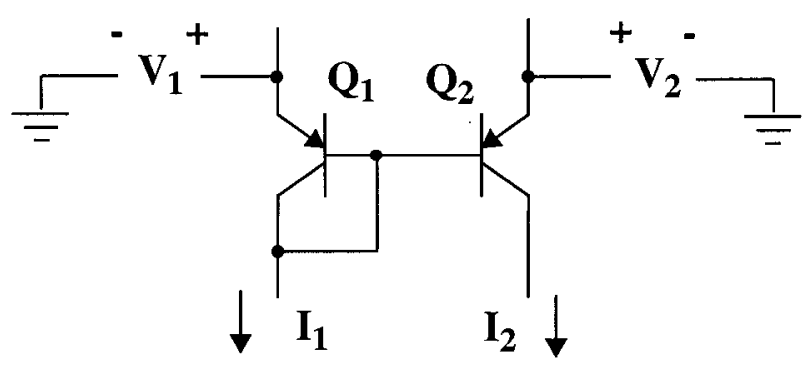

Figure 3.1: The basic two-transistor log-domain cell 
By expressing $I_{C}$ as an exponential function of $V_{E B}$, and neglecting the effects of $\beta, V_{A}$, and $\mathrm{n}_{\mathrm{F}}$, Eqn. (3.1) can be rearranged to give

$$
V_{2}=V_{1}-v_{T} \cdot \ln \left(\frac{I_{1}}{I_{2}}\right)
$$

where $I_{1}$ and $I_{2}$ are as shown in Figure 3.1. Equation (3.2) can also be re-arranged as

$$
I_{2}=I_{1} e^{\frac{V_{2}-V_{1}}{v_{T}}} .
$$

Two special cases of this cell are shown below in Figure 3.2. From Eqn. (3.2), the output voltage $V_{\text {out }}$ for the circuit in Figure 3.2(a) can be expressed as

$$
V_{\text {out }}=-v_{T} \cdot \ln \left(\frac{I_{o}+I_{\text {in }}}{I_{o}}\right)
$$

while from Eqn. (3.3), the current $I_{\text {out }}$ for the circuit in Figure 3.2(b) can be expressed as

$$
I_{\text {out }}=I_{o} e^{\frac{-V_{\text {in }}}{v_{T}}}-I_{o} .
$$
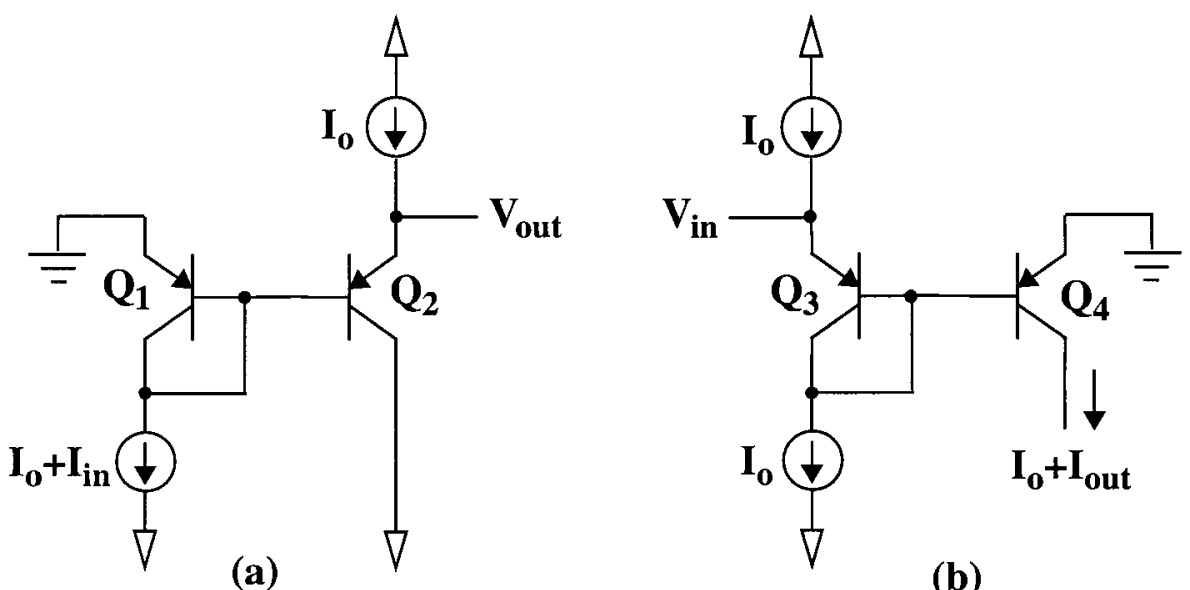

(b)

Figure 3.2: Two special cases demonstrating the $\operatorname{LOG}(x)$ and the $\operatorname{EXP}(x)$ operations respectively 
From these, two complementary mathematical operators can be defined:

$$
\operatorname{LOG}(x)=-v_{T} \cdot \ln \left(\frac{I_{o}+x}{I_{o}}\right)
$$

and

$$
\operatorname{EXP}(x)=I_{o} e^{\frac{-x}{v_{T}}}-I_{o}
$$

These functions imitate logarithmic and exponential operations, and can be implemented directly using the circuitry shown in Figure 3.2. In fact, the circuit in Figure 3.2(a) will be used to directly implement a logarithmic compression stage, and the circuit in Figure 3.2(b) will be used to directly implement an exponential expansion stage, each as shown in the block diagram of Figure 1.2 in Chapter 1. Note also that the operations defined in Eqns. (3.6) and (3.7) will be used throughout the next two chapters, and will form the mathematical language used to describe and develop log-domain circuits throughout this work.

With the $\operatorname{LOG}(\mathrm{x})$ and $\operatorname{EXP}(\mathrm{x})$ functions and associated circuitry defined, the development of a log-domain integrator can now be described.

\subsection{2 - The Log-Domain Integrator}

A PNP-only log-domain integrator is shown below in Figure 3.3. The cell has been adapted from a low voltage NPN-only integrator described in [1], [11]. The circuit below has two inputs, labelled as $\hat{V}_{p}$ and $\hat{V}_{n}$, and an output labelled as $\hat{V}_{\text {out }}{ }^{1}$. The integrator is implemented using two translinear loops. The first loop (from $\hat{V}_{p}$ to $\hat{V}_{\text {out }}$ ) is formed by $\mathrm{Q}_{1}$ and $\mathrm{Q}_{2}$, while the second loop (from $\hat{V}_{n}$ to $\hat{V}_{\text {out }}{ }^{\prime}$ ) is formed by $\mathrm{Q}_{3}$ and $\mathrm{Q}_{4}$. A simple voltage buffer from $\hat{V}_{\text {out }}$ to $\hat{V}_{\text {out }}$ is formed by $\mathrm{Q}_{5}$ and $\mathrm{Q}_{6}$, while the current pulled into the $\hat{V}_{\text {out }}$ ' node is mirrored into the $\hat{V}_{\text {out }}$ node by $\mathrm{Q}_{7}$ and $\mathrm{Q}_{8}$. A more detailed analysis of the circuit is provided below.

1. The "^" mark has been used to designate voltages that represent signals which have undergone logarithmic compression. 


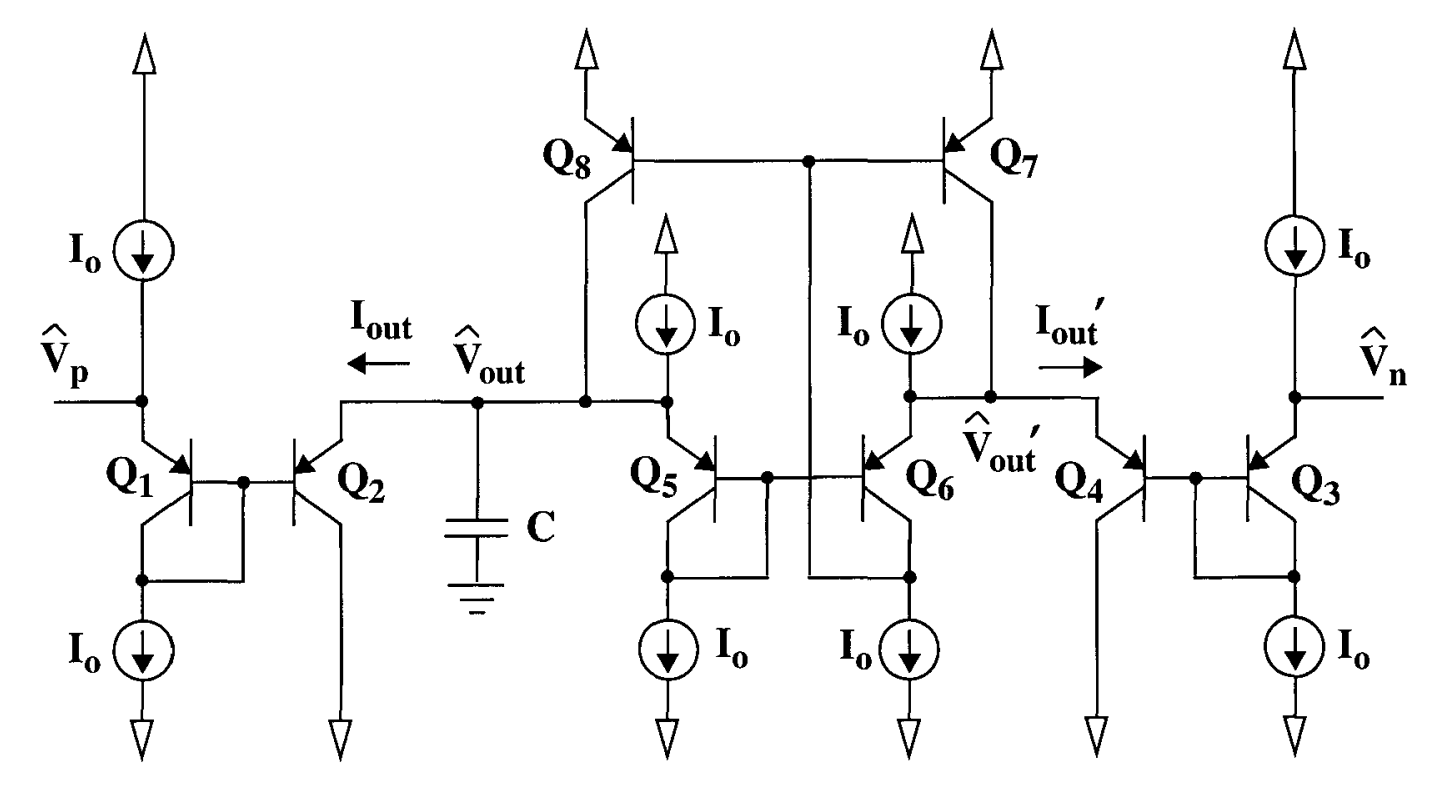

Figure 3.3: A PNP-only log-domain integrator

To derive the transfer function of the circuit, an expression relating the voltages around each translinear loop must be determined. For the first loop the output current can be expressed in terms of Eqn. (3.3), given by

$$
I_{\text {out }}=I_{o} e^{\frac{\hat{V}_{\text {out }}-\hat{V}_{p}}{v_{T}}}
$$

where $I_{0}$ is the bias current in $Q_{1}$ and $I_{o u t}$ is the bias current in $Q_{2}$, as shown in Figure 3.3. For the second loop, a similar expression can be derived, given by

$$
I_{\text {out }}{ }^{\prime}=I_{o} e^{\frac{\hat{V}_{\text {out }}-\hat{V}_{n}}{v_{T}}}
$$

A voltage buffer, formed by $\mathrm{Q}_{5}$ and $\mathrm{Q}_{6}$, has been inserted between the two loops. Each transistor is biased with a constant current such that each $V_{E B}$ is fixed. The signal $\hat{V}_{\text {out }}$ at the emitter of $\mathrm{Q}_{5}$ will be level shifted the base of $\mathrm{Q}_{5}$ and $\mathrm{Q}_{6}$, and then replicated at the emitter of $\mathrm{Q}_{6}, \hat{V}_{\text {out }}{ }^{\prime}$. Therefore the voltage $\hat{V}_{\text {out }}$ ' will be equal to $\hat{V}_{\text {out }}$. This is achieved without loading the node $\hat{V}_{\text {out }}$. 
A current mirror, formed by $\mathrm{Q}_{7}$ and $\mathrm{Q}_{8}$, provides a means of subtracting the two output currents $I_{\text {out }}$ and $I_{\text {out }}{ }^{\prime}$. The current drawn from the $\hat{V}_{\text {out }}{ }^{\prime}$ by $\mathrm{Q}_{4}$ will be pulled from $Q_{7}$, and since transistor $Q_{8}$ has the same $V_{E B}$ voltage as $Q_{7}$, the current will be mirrored, with a sign inversion, into the node $\hat{V}_{\text {out }}$. Note that the bases of $\mathrm{Q}_{7}$ and $\mathrm{Q}_{8}$ are connected to the collector of $\mathrm{Q}_{6}$, ensuring that the voltages at collectors of $\mathrm{Q}_{7}$ and $\mathrm{Q}_{8}$ are both floating and free to follow the voltages at the $\hat{V}_{\text {out }}$ and $\hat{V}_{\text {out }}{ }^{\prime}$ nodes.

Combining the voltage buffering and current mirroring of transistors $\mathrm{Q}_{5}-\mathrm{Q}_{8}$, the sum of the currents flowing at the output node, $\hat{V}_{\text {out }}$, can be expressed as

$$
-C \cdot \frac{d \hat{V}_{\text {out }}}{d t}=I_{o} e^{\frac{\hat{V}_{\text {out }}-\hat{V}_{p}}{v_{T}}}-I_{o} e^{\frac{\hat{V}_{\text {out }}-\hat{V}_{n}}{v_{T}}} .
$$

Applying the chain rule, the derivative term can be expanded, and the equation rewritten as

$$
C \cdot \frac{v_{T}}{I_{o}} \cdot e^{\frac{\hat{V}_{o u t}}{v_{T}}} \cdot \frac{d}{d t}\left(I_{o} e^{\frac{-\hat{V}_{o u t}}{v_{T}}}\right)=I_{o} e^{\frac{\hat{V}_{o u t}-\hat{V}_{p}}{v_{T}}}-I_{o} e^{\frac{\hat{V}_{o u t}-\hat{V}_{n}}{v_{T}}} .
$$

By multiplying through by $e^{\frac{-\hat{V}_{\text {out }}}{v_{T}}}$ and then subtracting the term $\mathrm{I}_{\mathrm{O}}$ from each exponential expression, the equation can be written in form

$$
C \cdot \frac{v_{T}}{I_{o}} \cdot \frac{d}{d t}\left(I_{o} e^{\frac{-\hat{V}_{\text {out }}}{v_{T}}}-I_{o}\right)=\left(I_{o} e^{\frac{-\hat{V}_{p}}{v_{T}}}-I_{o}\right)-\left(I_{o} e^{\frac{-\hat{V}_{n}}{v_{T}}}-I_{o}\right)
$$

Using the mapping defined in Eqn. (3.7), the expression can be rewritten as

$$
\operatorname{EXP}\left(\hat{V}_{\text {out }}\right)=\frac{I_{o}}{v_{T}} \cdot \frac{1}{C} \cdot \int\left\{\operatorname{EXP}\left(\hat{V}_{p}\right)-\operatorname{EXP}\left(\hat{V}_{n}\right)\right\} d t
$$

Therefore, in terms of the logarithmic and exponential operations defined in the previous section, the circuit in Figure 3.3 can be used to implement a log-domain integrator. 


\subsection{3 - The Damped Log-Domain Integrator}

An important variation of the above log-domain integrator is the damped integrator, which can be implemented by connecting the output of the standard integrator,

$\hat{V}_{\text {out }}$, to the negative input, $\hat{V}_{n}$. A symbolic representation for the standard integrator and the damped integrator are shown Figure 3.4(a) and (b) respectively. The circuit equation for a damped integrator, as given by Eqn. (3.10), can be simplified to

$$
C \cdot \frac{d \hat{V}_{\text {out }}}{d t}=I_{o} e^{\frac{\hat{V}_{p}-\hat{V}_{\text {out }}}{v_{T}}}-I_{o} .
$$

Notice that the damped integrator circuit can be realized by replacing transistors $\mathrm{Q}_{3}-\mathrm{Q}_{8}$ in Figure 3.3 by a constant current source, $\mathrm{I}_{\mathrm{o}}$, as shown in Figure 3.4(c). The transfer function of the damped integrator can be described by

$$
\operatorname{EXP}\left(\hat{V}_{\text {out }}\right)=\frac{I_{o}}{v_{T}} \cdot \frac{1}{C} \cdot \int\left\{\operatorname{EXP}\left(\hat{V}_{p}\right)-\operatorname{EXP}\left(\hat{V}_{\text {out }}\right)\right\} d t,
$$

which will be stable at all frequencies, unlike an undamped integrator, which is theoretically unstable in the presence of a DC input. The damped integrator circuit will be used for simulations and experimental measurements of the integrator performance provided later in this chapter.
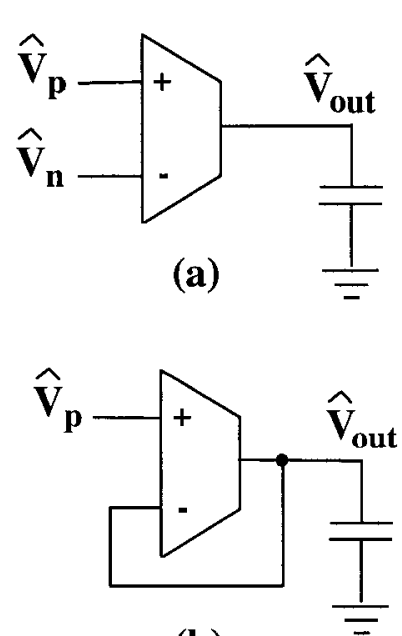

(b)

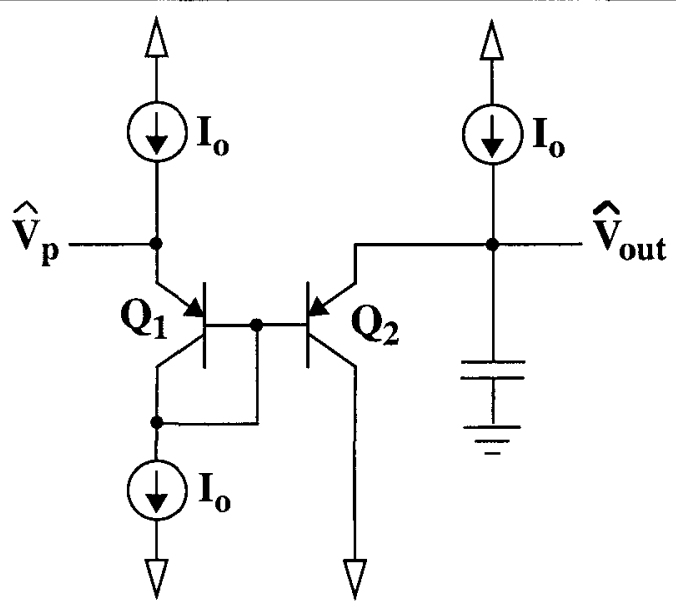

(c)

Figure 3.4: (a) Symbolic representation of an integrator (b) Symbolic representation of a damped integrator (c) A reduced realization of a PNP-only damped integrator circuit 


\section{2 - The CMOS Log-Domain Integrator}

The following section will describe the methodology used in mapping the bipolar log-domain integrator into a CMOS process. Several considerations, including issues surrounding the increase in circuit complexity and the circuit power consumption, will be discussed. In addition, the supporting circuitry including logarithmic compression and exponential expansion circuitry will be described at the end of this section.

\subsection{1 - Mapping the Log-Domain Integrator in CMOS Technology}

In order to develop a log-domain integrator in CMOS technology, first note that the lateral PNP transistors could be used to directly implement the PNP-only integrator presented in Section 3.1. This circuit, included here for comparative purposes, is shown in Figure 3.5(a).

To improve upon this design, observe that only the translinear loops $\left(\mathrm{Q}_{1}, \mathrm{Q}_{2}, \mathrm{Q}_{3}\right.$ and $\mathrm{Q}_{4}$ ) need to be implemented with bipolar transistors, and that PMOS transistors (operating in strong inversion) could be used to implement remaining circuitry. It follows that when working in a CMOS process which has been optimized for MOS performance (and which provides rigorous MOSFET models), PMOS transistors should be used in place of lateral PNP transistors when possible. In addition, since the Early voltage of the lateral PNP devices is relatively low, PMOS transistors can be used to provide cascoding, which has been shown through simulation to significantly improve the integrator performance. Further improvements can also be realized by placing level shifting PMOS transistors in the diode-connected devices $\left(\mathrm{Q}_{1}, \mathrm{Q}_{3}\right.$, and $\mathrm{Q}_{6}$ in Figure 3.5(a)), which serve not only to keep cascode transistors from operating in triode, but also to provide DC beta compensation for the bipolar devices in each of the translinear loops. The improved logdomain integrator is shown in Figure 3.5(b). 


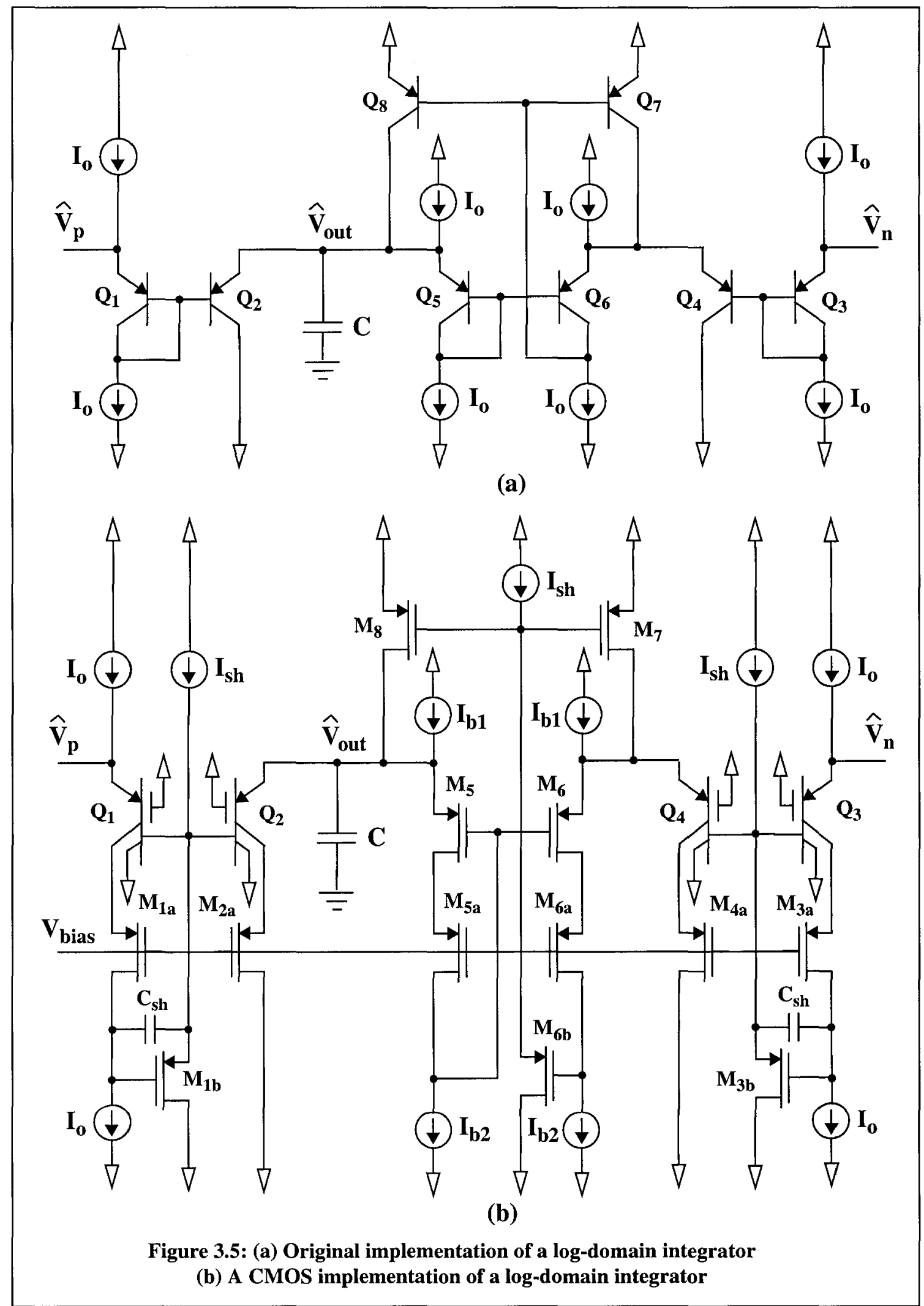


While the original circuit in Figure 3.5(a) was biased with a universal value of current, designated by $\mathrm{I}_{0}$, the improved circuit in Figure 3.5(b) has been biased with four independent currents, designated by $\mathrm{I}_{\mathrm{o}}$ (the bias current for the translinear loop), $\mathrm{I}_{\mathrm{b} 1}$ and $I_{b 2}$ (the bias current of the voltage/current buffer) and $I_{s h}$ (the bias current for the level shifter). The reason for this change stems from the replacement of bipolar transistors by MOSFETs in the improved design. While the lateral bipolar transistors can operate over a range of several decades of current, the MOSFET transistors (particularly those associated with $\mathrm{Q}_{5}-\mathrm{Q}_{8}$ ) have not been intended to be biased at low levels of current, at which point they enter the subthreshold mode of operation. For this reason, $\mathrm{I}_{\mathrm{b} 1}$ and $\mathrm{I}_{\mathrm{b} 2}$ are generally not dropped below a few microamps, and need to be controlled independently when the translinear loop bias current is dropped to $1 \mu \mathrm{A}$ and below. In addition, the difference between these bias currents $\left(\mathrm{I}_{\mathrm{b} 2}-\mathrm{I}_{\mathrm{b} 1}\right)$ can be used to ensure a constant current bias of a few microamps in $\mathrm{M}_{7}$ and $\mathrm{M}_{8}$. Finally, the remaining bias current $\mathrm{I}_{\mathrm{sh}}$ is used to control the gate-source voltage of the level shifting transistors associated with $\mathrm{Q}_{1}, \mathrm{Q}_{3}$, and $\mathrm{Q}_{5}$. This current is generally kept at a bias value of a few microamps. Note that the bias currents themselves have been generated using current mirrors implemented entirely with MOSFET transistors.

Circuit complexity issues aside, the most significant drawback to the modifications made to the integrator in Figure 3.5(b) come in the form of increased power consumption. The increase is due to two factors: firstly, because of the use of cascoding and the relatively large threshold voltage of the PMOS transistors (approximately $0.8 \mathrm{~V}$ in the $0.35 \mu$ CMOS process used in this work) the circuit must operate from a higher voltage supply than in the initial implementation. While its original bipolar technology counterpart was capable of operation down to $1 \mathrm{~V}$ [8], the circuit in Figure 3.5(b)has been designed to operate from a $2.5 \mathrm{~V}$ supply, and was found not to perform well at supplies below $2.2 \mathrm{~V}$. The second factor leading to increased power consumption stems from the fact that the values of $\mathrm{I}_{\mathrm{b} 1}, \mathrm{I}_{\mathrm{b} 2}$, and $\mathrm{I}_{\mathrm{sh}}$ are not generally not scaled in proportion to $\mathrm{I}_{\mathrm{o}}$ for low levels of bias current, as has been described above. Therefore, the circuit in Figure 3.5(b) is not as efficient as its predecessor for low-power applications. 
An additional comment should be made regarding the capacitance $\mathrm{C}_{\mathrm{sh}}$, as shown in Figure 3.5(b). At low levels of $\mathrm{I}_{0}$, a resonance in the integrator response can occur due to feedback in the loop formed by $\mathrm{Q}_{1}, \mathrm{M}_{1 \mathrm{a}}$, and $\mathrm{M}_{1 \mathrm{~b}}$ in the level shifting circuitry (and similarly for $\mathrm{Q}_{3}, \mathrm{M}_{3 \mathrm{a}}$ and $\mathrm{M}_{3 \mathrm{~b}}$ ). The resonance can be reduced or eliminated by inserting $\mathrm{a}$ small additional capacitance $(\leq 1 \mathrm{pF})$ into this loop, as shown by $\mathrm{C}_{\mathrm{sh}}$, without reducing the bandwidth of the integrator. The inclusion of this capacitance, though not strictly required, can improve the ideality of the frequency response of the integrator at the expense of additional layout area. This trade-off could be a particularly important consideration if capacitances as large as $1 \mathrm{pF}$ are used for each $\mathrm{C}_{\mathrm{sh}}$. The circuitry described in the experimental results section of this work utilized $\mathrm{C}_{\mathrm{sh}}=1 \mathrm{pF}$. No such capacitance was included in the MOS-only loop formed by $M_{6}, M_{6 a}, M_{6 b}$, and $M_{7}$.

The final design of the log-domain integrator, implemented in $0.35 \mu$ CMOS technology, is shown in Figure 3.5(b) with transistor dimensions as given in Table 3.1. All devices were reduced to the minimum gate length in order maximize the potential operating speed of the circuit, with the exception of $M_{7}$ and $M_{8}$, which were kept at a gate length of $0.8 \mu$ since these devices were not cascoded.

The integrator conforms to the integrator equation given by Eqn. (3.13) and the simulated and measured responses will be discussed in detail in Section 3.3. Before presenting these results, a description of the logarithmic compression and exponential expansion circuitry will be provided.

Table 3.1: Transistor Dimensions in CMOS Log-Domain Integrator Circuit

\begin{tabular}{|c|c|}
\hline Transistor & Transistor Dimensions \\
\hline \hline $\mathrm{Q}_{1}, \mathrm{Q}_{2}, \mathrm{Q}_{3}, \mathrm{Q}_{4}$ & $\begin{array}{c}2 \times \text { unit size lateral PNP transistor } \\
\text { (shown in Figure 2.3) }\end{array}$ \\
\hline $\mathrm{M}_{1 \mathrm{a}}, \mathrm{M}_{2 \mathrm{a}}, \mathrm{M}_{3 \mathrm{a}}, \mathrm{M}_{4 \mathrm{a}}$ & $4 \times(3 \mu \times 0.35 \mu)$ \\
\hline $\mathrm{M}_{1 \mathrm{~b}}, \mathrm{M}_{3 \mathrm{~b}}$ & $4 \times(12 \mu \times 0.35 \mu)$ \\
\hline $\mathrm{M}_{5}, \mathrm{M}_{5 \mathrm{a}}, \mathrm{M}_{6}, \mathrm{M}_{6 \mathrm{a}}$ & $4 \times(6 \mu \times 0.35 \mu)$ \\
\hline $\mathrm{M}_{6 \mathrm{~b}}$ & $8 \times(12 \mu \times 0.35 \mu)$ \\
\hline $\mathrm{M}_{7}, \mathrm{M}_{8}$ & $4 \times(12 \mu \times 0.8 \mu)$ \\
\hline
\end{tabular}




\subsection{2 - Input Compression / Output Expansion and Interface Circuitry}

In order to interface log-domain filter circuits to the outside (linear, as opposed to logarithmic) world, additional supporting circuitry is required. First, since log-domain filters are essentially current-mode circuits, a means must be provided to convert linear currents into the logarithmically compressed voltages, and in turn to convert logarithmically compressed voltages to linear currents. In addition, since it is most convenient to interface filters to test equipment using voltages rather than currents, a means should be included to provide linear voltage to current (V/I) conversion at the filter input and linear current to voltage (I/V) conversion at the filter output. Each of these four functions can be realized in a simple and compact manner using the circuitry described in this section.

A CMOS implementation of the logarithmic compression circuit, originally given in Figure 3.2(a), is shown below in Figure 3.6(a). In this circuit, the emitter of $Q_{1}$ is grounded and an input current, $\mathrm{I}_{\mathrm{in}}$, is introduced at the drain of $\mathrm{M}_{1 \mathrm{a}}$. The input current is pulled through $\mathrm{Q}_{1}$ and is logarithmically compressed in the form of the voltage at the emitter of $\mathrm{Q}_{2}$, as was previously derived in Eqn. (3.4). This voltage can be expressed in mathematical notation, according to the operator defined in Eqn. (3.6), as $\hat{V}_{i}=\operatorname{LOG}\left(I_{i n}\right)$. Note also that this circuitry can be implemented directly in place of the positive (or negative) translinear loops of the log-domain integrator itself.

A CMOS implementation of the exponential expansion circuit, is shown in Figure 3.6(b). In this circuit, the emitter of $Q_{4}$ is grounded and a logarithmically compressed input voltage is introduced at the emitter of $\mathrm{Q}_{3}$. The difference in voltages at the emitters of $\mathrm{Q}_{3}$ and $\mathrm{Q}_{4}$ lead to an output current, $\mathrm{I}_{\text {out }}$, which represents an exponential expansion of the applied input voltage, as was previously derived in Eqn. (3.5). This current can be expressed in mathematical notation, according the operator defined in Eqn. (3.6), as $I_{\text {out }}=\operatorname{EXP}\left(\hat{V}_{i}\right)$. In addition, by including a current source at the emitter of $\mathrm{Q}_{3}$, as shown in Figure 3.6(b), the circuit can be used to exponentially expand the voltage at any node without loading the node itself. The transistor dimensions for both the input compression and output expansion circuits are as given previously in Table 3.1 
It is important to note that the two circuits shown in Figure 3.6 can be used within any portion of the implementation of a log-domain filter or circuit. In fact, as will be seen in Chapter 4, the derivation of both biquadratic and LC-ladder based log-domain filters are based upon the original assumption that each integrator block is preceeded by a logarithmic compression stage and immediately followed by an exponential expansion stage. However, as will become apparent when a signal flow graph approach is applied, a natural cancellation of the logarithmic and exponential operations can be made to occur during the filter design process, leaving the entire filter with only a single compression stage at its input and a single expansion stage at its output. The filter will therefore conform to the block diagram presented in Figure 1.2 in Chapter 1, in which only single compression and expansion stages are present.

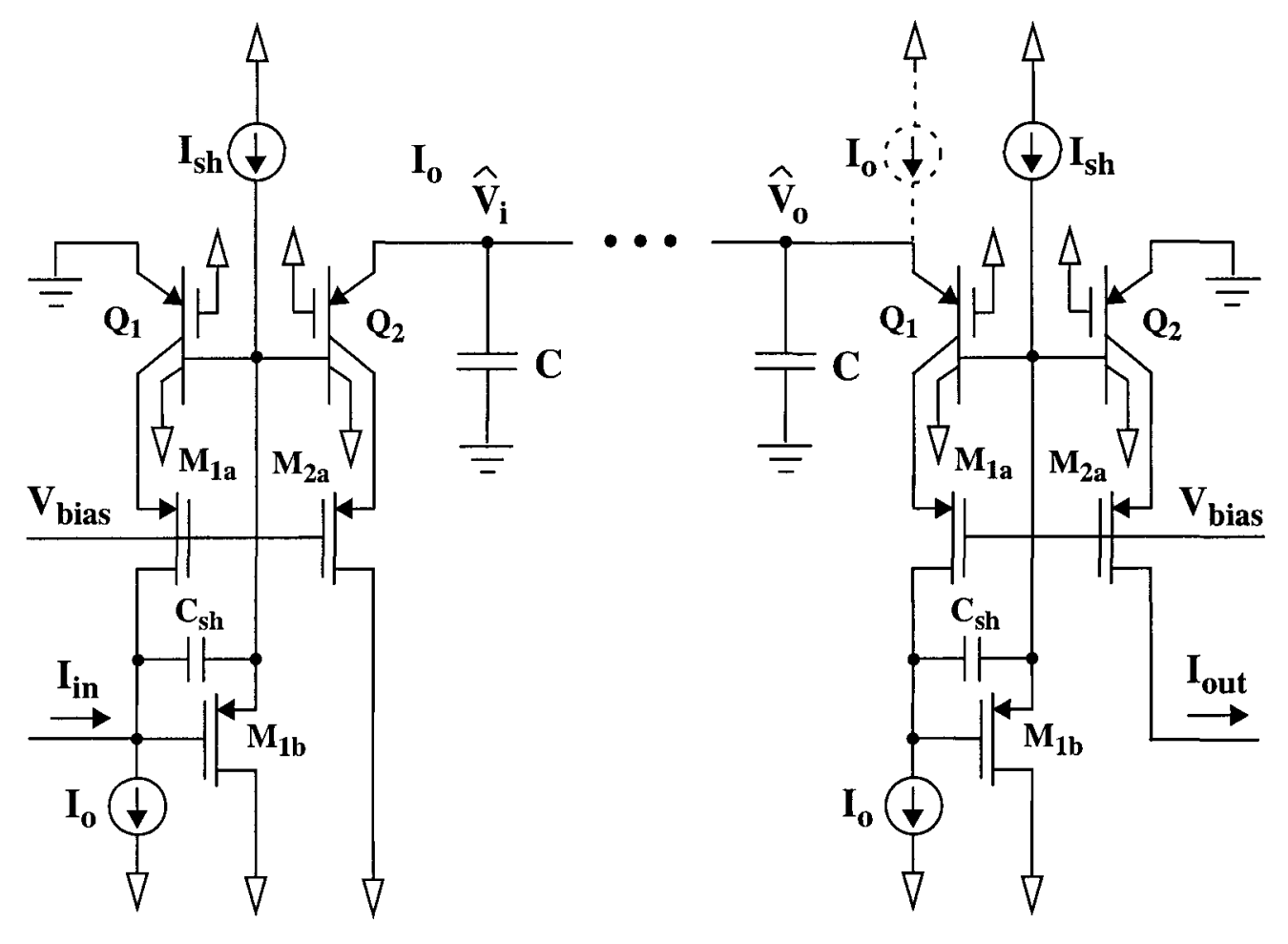

(a) $\hat{V}_{i}=\operatorname{LOG}\left(I_{i n}\right)$

(b) $I_{\text {out }}=\operatorname{EXP}\left(\hat{V}_{o}\right)$

Figure 3.6: (a) A CMOS implementation of a logarithmic compression circuit (b) A CMOS implementation of an exponential expansion circuit 
A compact all-CMOS implementation of a V/I converter, adapted from [11], is shown in Figure 3.7. In this circuit, transistors $\mathrm{M}_{1}$ and $\mathrm{M}_{2}$ are each biased with a constant current $I_{v i}$, while the diode connection between the gate of $M_{2}$ and the drain of $M_{2 a}$ provide a reference voltage to the gate of $\mathrm{M}_{1}$. This arrangement ensures that the source of $\mathrm{M}_{1}$ will be biased to DC ground. Furthermore, the feedback path formed by transistors $M_{1}, M_{1 a}, M_{3}$ (and the DC level shifter $M_{4}$ ) serve to decrease the impedance seen at the source of M1 such that the node also serves as a virtual signal ground. This allows the V/I conversion to be implemented with a resistor, $R_{1}$, as shown in the figure.

To deliver this current to the desired log-domain circuit, note that the signal at the input will be first shunted through $\mathrm{M}_{3}$, then replicated by $\mathrm{M}_{5}$. The current will be forced through $\mathrm{M}_{5 \mathrm{a}}$, reaching the output node at the drain of $\mathrm{M}_{5 \mathrm{~b}}$. The impedance of this node is high due to the use of cascoding, and the DC voltage at the node is compatible with the input logarithmic compression stage shown in Figure 3.6.

In this way, a simple circuit can be used to provide the required input V/I conversion at the input of the filter. Since the constant of voltage conversion is directly set by $R_{1}$, the input current can be readily scaled to an appropriate level. In addition, the output $\mathrm{I} / \mathrm{V}$ conversion can be implemented in a very simple manner by connecting the exponential expansion stage, as shown in Figure 3.6(b), to a resistor $R_{2}$ at its output. In this case, the overall gain of the filter can be conveniently set using the ratio $\left(R_{2} / R_{1}\right)$. The dimensions for the transistors shown in Figure 3.7 are provided below in Table 3.2.

Table 3.2: Transistor dimensions for compression and expansion circuits

\begin{tabular}{|c|c|}
\hline Transistor & Transistor Dimensions \\
\hline $\mathrm{M}_{1}, \mathrm{M}_{1 \mathrm{a}}, \mathrm{M}_{2}, \mathrm{M}_{2 \mathrm{a}}, \mathrm{M}_{4 \mathrm{a}}, \mathrm{M}_{4 \mathrm{~b}}$ & $4 \times(6 \mu \times 0.35 \mu)$ \\
\hline $\mathrm{M}_{3}, \mathrm{M}_{4}$ & $8 \times(12 \mu \times 0.8 \mu)$ \\
\hline $\mathrm{M}_{5}$ & $8 \times(12 \mu \times 0.35 \mu)$ \\
\hline
\end{tabular}




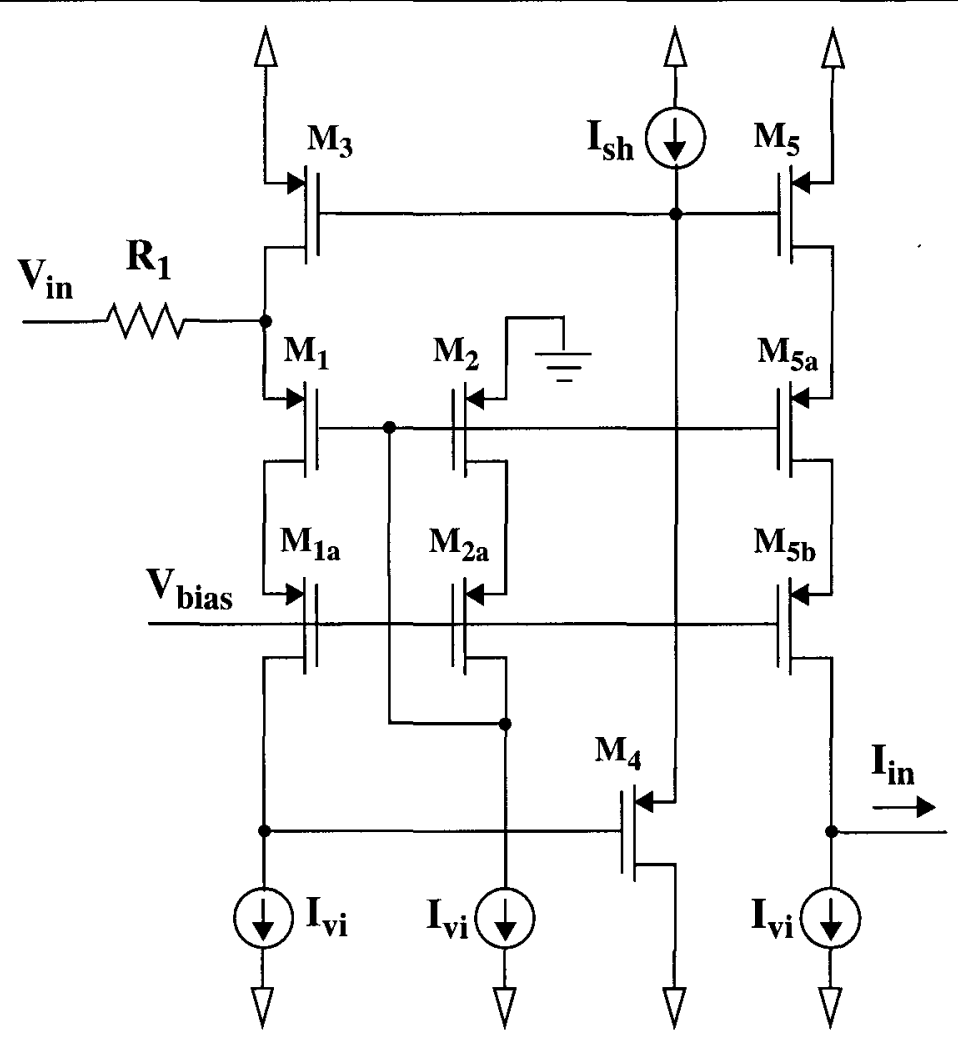

Figure 3.7: (a) A CMOS compatible V/I converter

The circuitry presented in this section can be used to implement a complete logdomain integrator, and the remainder of the chapter will be devoted to the simulated and experimental characterization of this integrator. It will be demonstrated that these circuits have promising performance characteristics, and by extension that the lateral PNP transistor can be used to implement useful circuits in CMOS technology.

\section{3 - Simulated and Experimental Results}

The final section of this chapter will discuss the simulated and experimental performance of a log-domain integrator fabricated in $0.35 \mu$ CMOS technology. Three categories of tests have been used to characterize the integrator performance: frequency response, linearity and distortion performance, and noise performance. Each will be described in detail, and a summary of performance will be provided at the end of the chapter. The section will begin with a description of the damped integrator circuit itself. 


\subsection{1 - Description of the Log-Domain Integrator Test Circuit}

The log-domain circuit under test is shown in Figure 3.8(a). Transistors used as cascode devices and level shifters have been omitted from the diagram for visual clarity, though all simulations and measurements have been made using the complete circuit implementations originally shown in Figures 3.5 - 3.7. The circuit is composed of an input V/I stage $\left(M_{1}, M_{3}, M_{4}\right.$ and $\left.R_{1}\right)$, a combined input compression and damped log-domain integrator stage $\left(Q_{1}, Q_{2}\right.$, and $\left.C\right)$, an output compression stage $\left(Q_{3}\right.$ and $\left.Q_{4}\right)$ and a $I / V$ converter (implemented with $\mathrm{R}_{2}$ ). As previously mentioned, the damped integrator circuit, rather than a two input integrator, was used for testing owing to its stability over all frequencies, unlike a standard integrator which is theoretically unstable in the presence of a DC input. Note that this circuit configuration does not allow for direct testing of the voltage/current buffer (transistors $\mathrm{M}_{5}-\mathrm{M}_{8}$ in Figure 3.5) within the integrator itself. However, initial simulations have clearly indicated that this portion of the circuitry does not impose significant limitations on the frequency response, linearity, or noise performance of the overall integrator. A symbolic representation of the test circuit is shown in Figure 3.8(b). The input compression stage in the diagram has incorporated into the integrator, as indicated in the figure.

In the testing which follows, integrating capacitors of $10 \mathrm{pF}$ (on chip), $100 \mathrm{pF}$ and 1 $\mathrm{nF}$ (placed off chip) were used, and the input and output resistors were set to $500 \Omega$ The current sources were implemented using PMOS and NMOS current mirrors, and the values of $I_{0}, I_{v i}$, and $I_{s h}$ could be independently controlled with off-chip references. For current biases from $I_{o}=10 \mu \mathrm{A}$ to $100 \mu \mathrm{A}$, the value of $\mathrm{I}_{\mathrm{vi}}$ was set to be equal to $\mathrm{I}_{\mathrm{o}}$, but for current biases with $\mathrm{I}_{\mathrm{o}}<10 \mu \mathrm{A}, \mathrm{I}_{\mathrm{vi}}$ was kept fixed at $10 \mu \mathrm{A}$. A value of $\mathrm{I}_{\mathrm{sh}}=5 \mu \mathrm{A}$ was used in all tests described in this section.

The power supplies used for the simulation and testing of this circuit were nominally set to $\mathrm{V}_{\mathrm{DD}}=2.5 \mathrm{~V}$, AGND (analog ground) $=2.0 \mathrm{~V}$, and $\mathrm{V}_{\mathrm{SS}}=0 \mathrm{~V}$. The measured frequency response of the log-domain filters was often found to be slightly improved by lowering $\mathrm{V}_{\mathrm{DD}}$ to $2.35 \mathrm{~V}$ to $2.45 \mathrm{~V}$, and most measurements were taken from this slightly lowered voltage. The bias voltage for the cascodes in both the integrator and the V/I circuit could be independently controlled, but was kept at $0.55 \mathrm{~V}$ above $\mathrm{V}_{\mathrm{SS}}$ for all tests which are described here. 
The log-domain circuit was manufactured in $0.35 \mu$ CMOS technology. All circuit simulations were performed using HSPICE and made use of the lateral PNP model developed in Chapter 2 as well as the $0.35 \mu$ models MOS provided by the manufacturer. All measurements were made using a Hewlett Packard 3588A Spectrum Analyzer, a Tektronix TDS800 Oscilloscope, and Hewlett Packard 3314A Function Generator. An off-chip unitygain buffer (an AD817 Op-Amp) was used between the filter output and the various test equipment.

A microphotograph of the chip is shown in Figure 3.9(a). The total die area for a single integrator (not including current sources) was $220 \mu \times 110 \mu$, while the total area for onchip (poly/poly) capacitors in this technology was $1700 \mu^{2} / \mathrm{pF}$. The circuit board used for testing is shown in Figure 3.9(b).

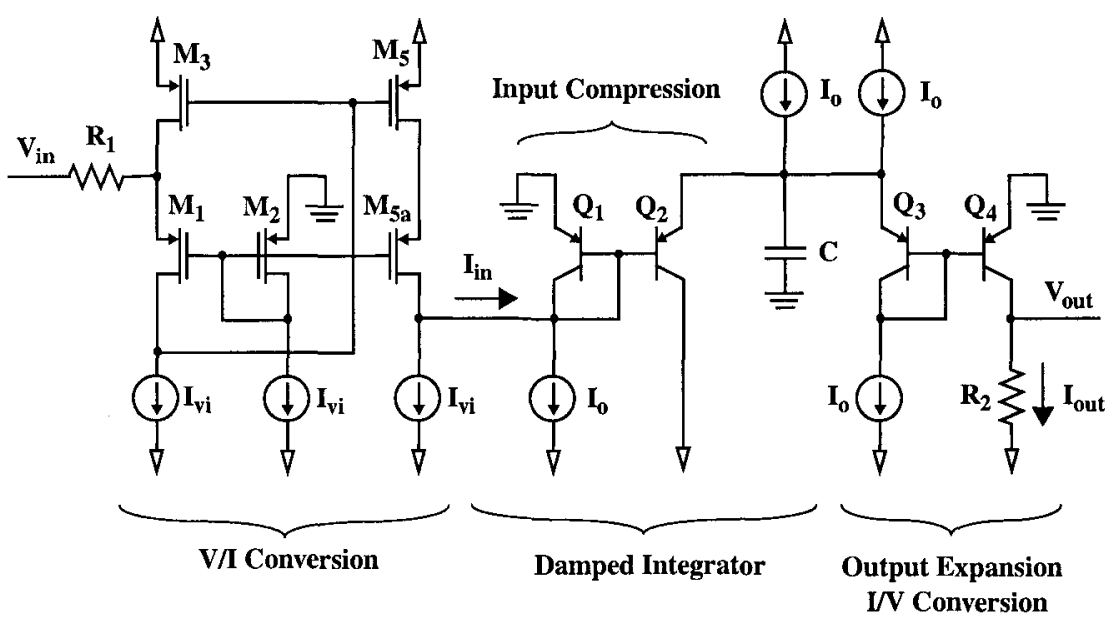

(a)

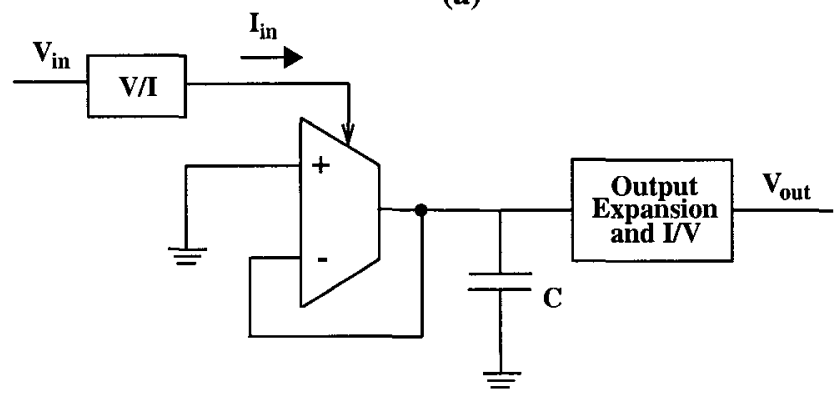

(b)

Figure 3.8: (a) A simplified schematic of the damped integrator circuit under test (b) A symbolic representation of the damped integrator circuit under test 


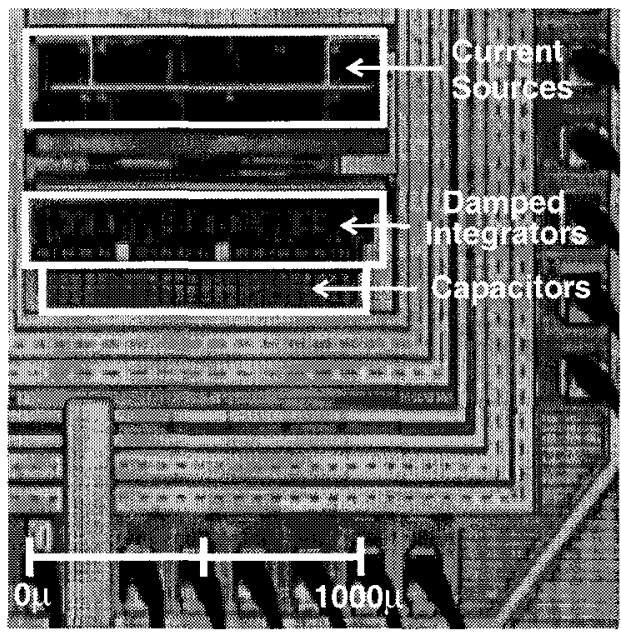

(a)

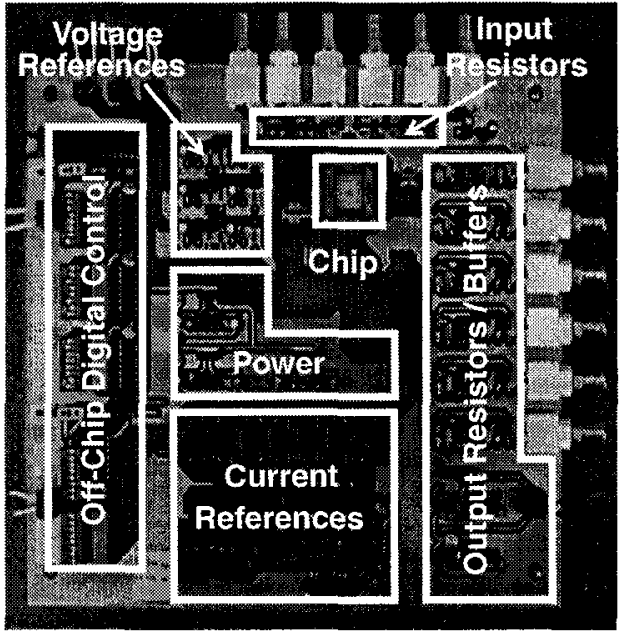

(b)

Figure 3.9: (a) A microphotograph of the damped integrators under test and associated circuitry (b) Photograph of the printed circuit board used to test the damped integrator

\subsection{2 - Frequency Response}

The first tests performed on the log-domain integrator measured the frequency response. Simulated and experimentally measured frequency responses for the integrator are shown in Figures 3.10 and 3.11. In the first figure, a constant capacitance of $10 \mathrm{pF}$ was used while the bias current $\mathrm{I}_{\mathrm{o}}$ was varied from $1 \mu \mathrm{A}$ to $100 \mu \mathrm{A}$. In the second figure, a constant current of $I_{o}=100 \mu \mathrm{A}$ was used while the capacitance was varied from $10 \mathrm{pF}$ to $1 \mathrm{nF}$. A summary of frequency performance of the integrator is provided in Table 3.5.

As previously mentioned, the damped integrator is, in effect, a first order low-pass filter. The proportionality between the $3-\mathrm{dB}$ frequency and the bias current $I_{0}$, and the inverse proportionality between the $3-\mathrm{dB}$ frequency and the integrating capacitor $\mathrm{C}$, is clearly demonstrated. These figures provide a verification that this CMOS integrator follows the log-domain relationships given by Eqn. (3.13) and (3.15) and further indicates that this CMOS lateral PNP transistor behaves in the manner expected of traditional bipolar devices. 


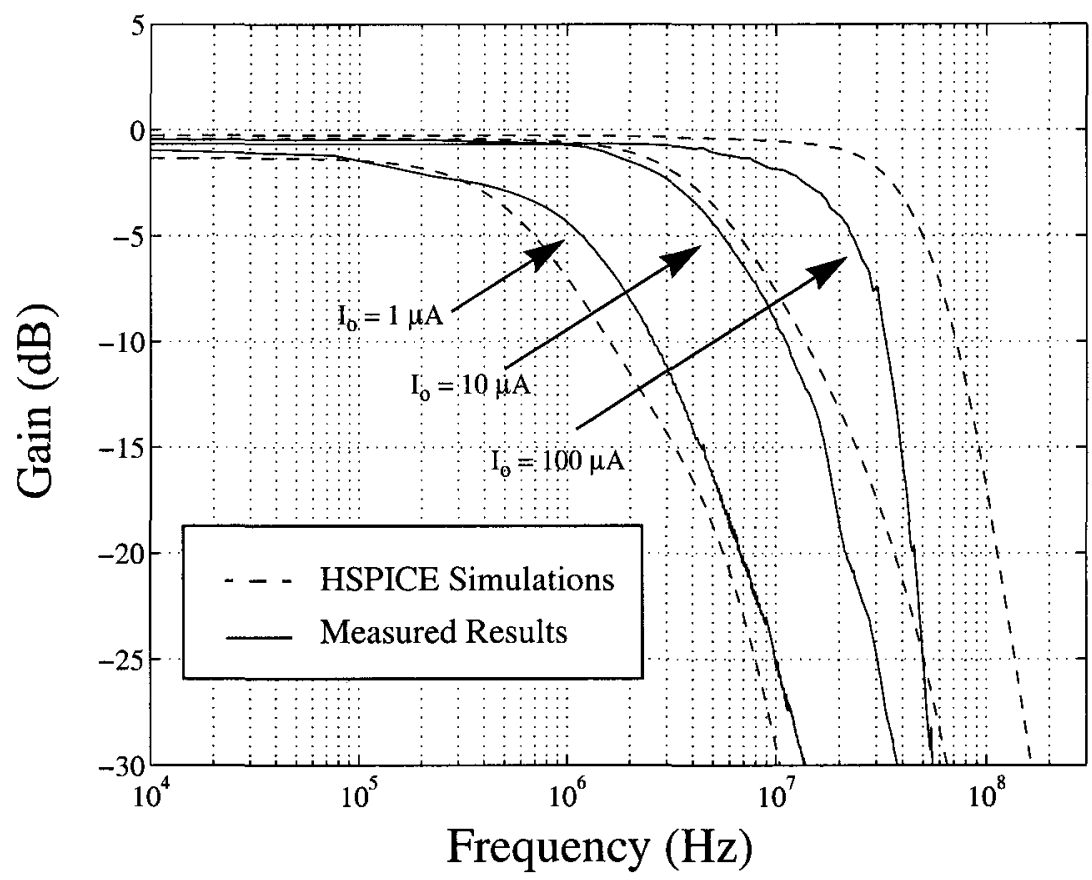

Figure 3.10: Frequency response of the $\log$-domain integrator, $C=10 \mathrm{pF}$.

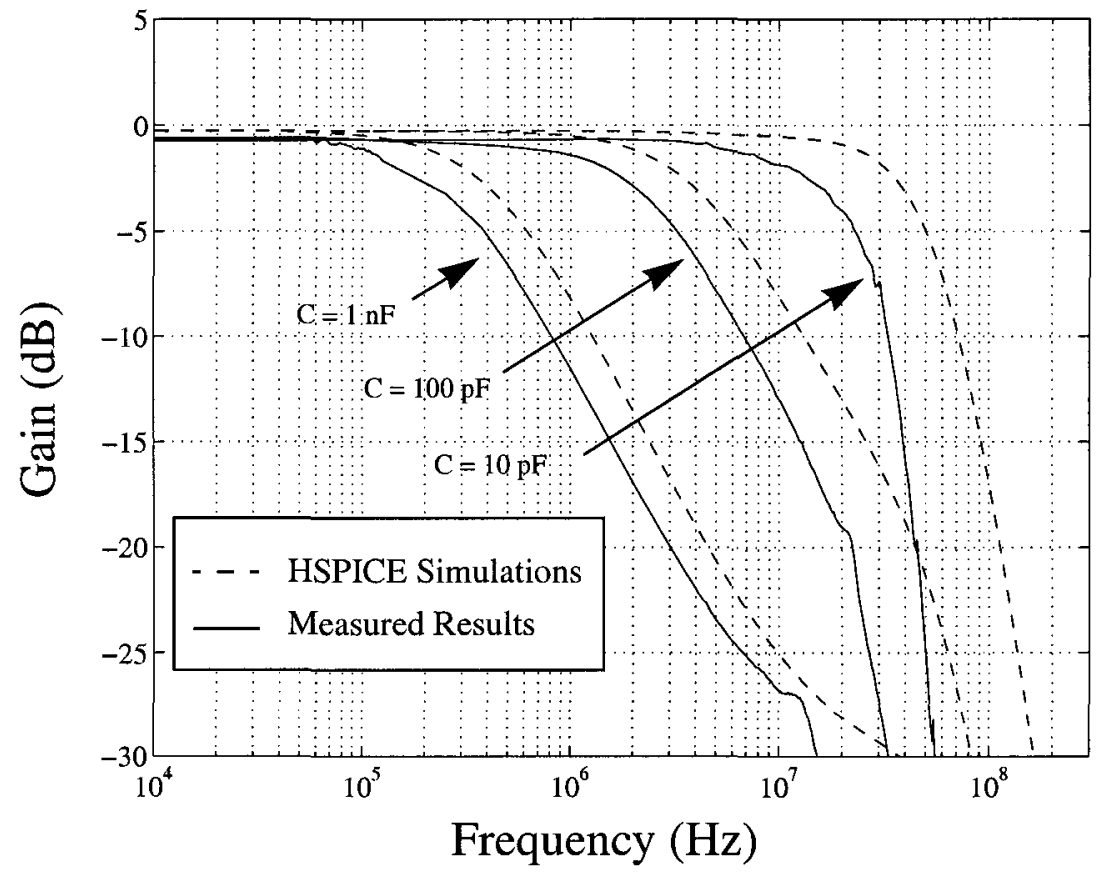

Figure 3.11: Frequency response of the log-domain integrator, $I_{0}=100 \mu \mathrm{A}$. 
The most significant deviations between the simulated and measured responses occurred at high bias levels and at high frequencies. At a bias current level of $\mathrm{I}_{\mathrm{o}}=100 \mu \mathrm{A}$, a significant drop in the measured frequency response was observed, and can be seen in both figures. Also note the increased slope of filter attenuation at high frequencies and high currents, reflecting the fact that the simulations did not adequately account for parasitic capacitances, both on the chip and on the circuit board. This deviation also points to the possibility that the frequency performance (specifically, the base transit time) of the lateral bipolar transistor has been underestimated during the device modelling.

These issues aside, it has been demonstrated that the 3-dB frequency of the filter can be tuned by almost two decades, and that an experimentally measured 3-dB frequency of 15 $\mathrm{MHz}$ or higher can be achieved with these devices.

\subsection{3 - Linearity and Distortion Performance}

All log-domain filter circuits suffer from distortion, due to part to non-idealities of the bipolar transistor being used. In the case of this CMOS log-domain integrator, distortion measurements are of particular interest since they provide an evaluation of the quality of the CMOS lateral bipolar transistors themselves. The distortion of this integrator has been measured in terms of two types of quantities: total harmonic distortion (THD), and the input and output third order intercept points (IIP3 and OIP3). A plot showing the simulated and experimental THD is shown in Figure 3.12 and a measurement demonstrating the calculation of the input and output intercept points is shown in Figure 3.13. Both measurements have been taken with $\mathrm{I}_{\mathrm{o}}=10 \mu \mathrm{A}$, and with an input signal of $100 \mathrm{kHz}$. Additional measurements were made with $\mathrm{I}_{\mathrm{o}}=50 \mu \mathrm{A}$, and the distortion performance from both sets of measurements is summarized in Table 3.3. Note that only single-tone distortion tests have been performed.

For the case where $\mathrm{I}_{\mathrm{o}}=10 \mu \mathrm{A}$, the experimentally measured THD at $\mathrm{I}_{\mathrm{in}}=0.5 \mathrm{I}_{\mathrm{o}}$ was $44.3 \mathrm{~dB}$. In general for $\mathrm{I}_{\mathrm{in}}<\mathrm{I}_{\mathrm{o}}$, the experimentally measured THD was close to, though a few decibels higher than, the THD predicted by simulation. At an increased bias current of $I_{0}=$ $50 \mu \mathrm{A}$, the experimentally measured THD at $\mathrm{I}_{\text {in }}=0.5 \mathrm{I}_{\mathrm{o}}$ was $-30.1 \mathrm{~dB}$, in close agreement with simulations values, but showing a significant drop from the $10 \mu \mathrm{A}$ case. 


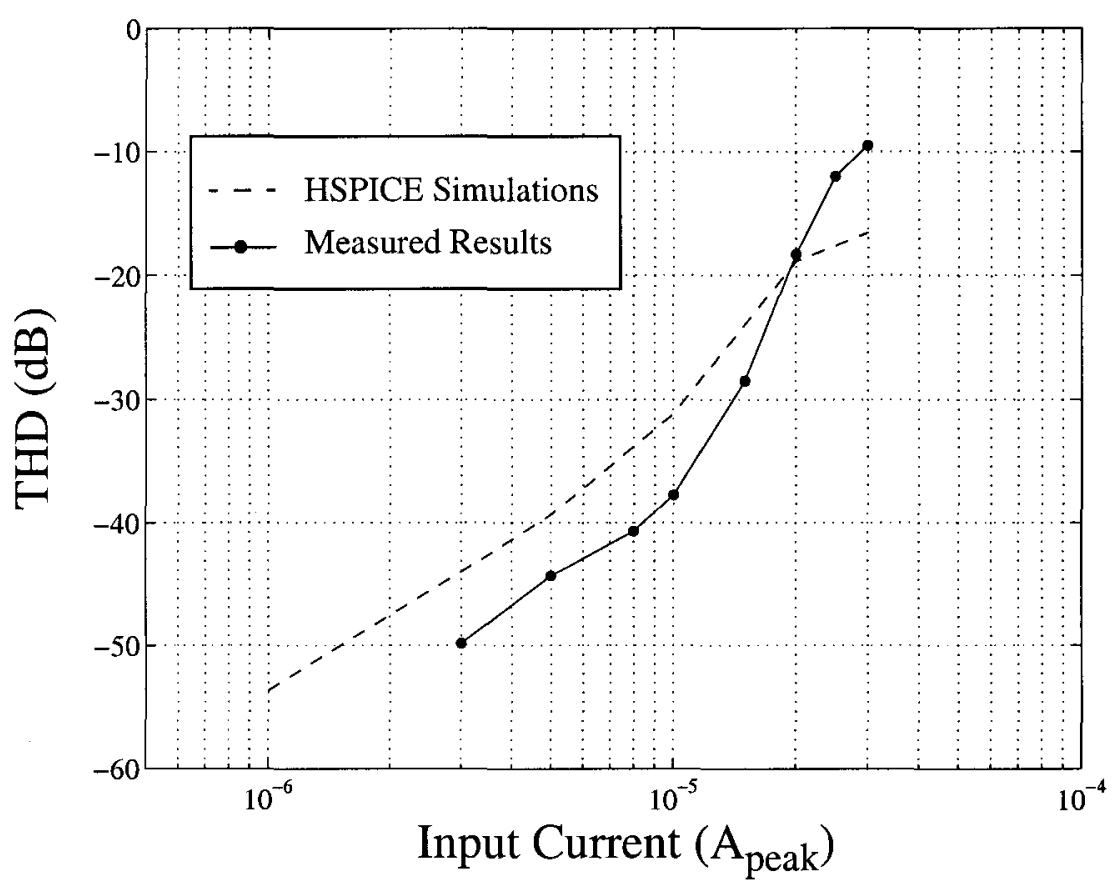

Figure 3.12: Total Harmonic Distortion, $I_{0}=10 \mu A, I_{\text {in }}=100 \mathrm{kHz}$

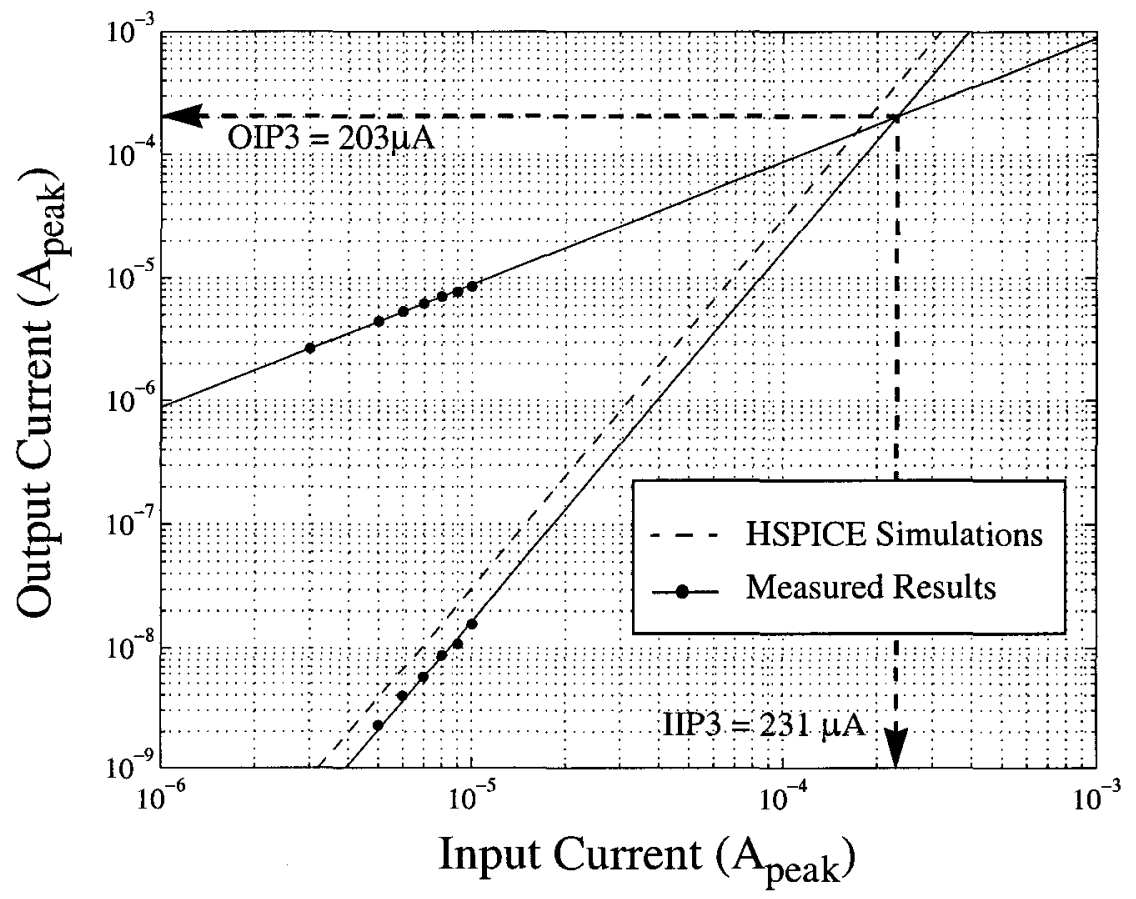

Figure 3.13: Third order intercept points, $I_{0}=10 \mu \mathrm{A}, I_{\mathrm{in}}=100 \mathrm{kHz}$ 
Table 3.3: Simulated and Measured Distortion Performance

\begin{tabular}{|c|c|c|c|}
\hline Parameter & Bias Current & $\begin{array}{c}\text { Simulation } \\
\text { Results }\end{array}$ & $\begin{array}{c}\text { Experimental } \\
\text { Results }\end{array}$ \\
\hline $\begin{array}{c}\text { Total Harmonic } \\
\text { Distortion } \\
\left(\mathrm{I}_{\text {in }}=0.5 \mathrm{I}_{\mathrm{o}}\right)\end{array}$ & $\mathrm{I}_{\mathrm{o}}=10 \mu \mathrm{A}$ & $-39.4 \mathrm{~dB}$ & $-44.3 \mathrm{~dB}$ \\
\cline { 2 - 4 } & $\mathrm{I}_{\mathrm{o}}=50 \mu \mathrm{A}$ & $-32.0 \mathrm{~dB}$ & $-30.1 \mathrm{~dB}$ \\
\hline $\begin{array}{c}\text { Third Order } \\
\text { Intercept } \\
\text { (OIP3) }\end{array}$ & $\mathrm{I}_{\mathrm{o}}=10 \mu \mathrm{A}$ & $180 \mu \mathrm{A}_{\text {peak }}$ & $203 \mu \mathrm{A}_{\text {peak }}$ \\
\cline { 2 - 4 } & $\mathrm{I}_{\mathrm{o}}=50 \mu \mathrm{A}$ & $670 \mu \mathrm{A}_{\text {peak }}$ & $439 \mu \mathrm{A}_{\text {peak }}$ \\
\hline
\end{tabular}

Further simulations have indicated that for bias currents above $50 \mu \mathrm{A}$, the circuit distortion increases significantly, since under such conditions the collector current begins to roll off due to high injection effects (see Figure 2.14) and therefore the transistors behave less like ideal exponential devices. For the case of $I_{0}=10 \mu \mathrm{A}$, the experimentally measured input and output third order intercepts were $231 \mu \mathrm{A}_{\text {peak }}$ and $203 \mu \mathrm{A}_{\text {peak }}$ respectively. As seen in Figure 3.13, these measurements are again close to, though slightly more favourable than, those predicted by simulation. At an increased bias of $\mathrm{I}_{\mathrm{O}}=50 \mu \mathrm{A}$, the experimentally measured input and output intercepts were $467 \mu \mathrm{A}_{\text {peak }}$ and $439 \mu \mathrm{A}_{\text {peak }}$ respectively, in this case slightly lower than the intercept points predicted by simulation.

In summary, the log-domain integrator exhibits reasonable linearity at low values of bias current, though distortion may place limitations on the maximum bias current levels (and therefore the bandwidths) at which the filter may be most useful.

\subsection{4 - Noise Performance}

The noise performance of the integrator is the final characteristic which has been examined. The noise performance of the CMOS integrator has been measured in terms of two quantities: the signal to noise ratio (SNR) and output noise current. Measurements of both quantities have again been made for $\mathrm{I}_{\mathrm{o}}=10 \mu \mathrm{A}$ and $\mathrm{I}_{\mathrm{o}}=50 \mu \mathrm{A}$. The SNR (as well as the $\mathrm{THD}$ ) for the case $\mathrm{I}_{\mathrm{o}}=10 \mu \mathrm{A}$ has been plotted in Figure 3.14. 


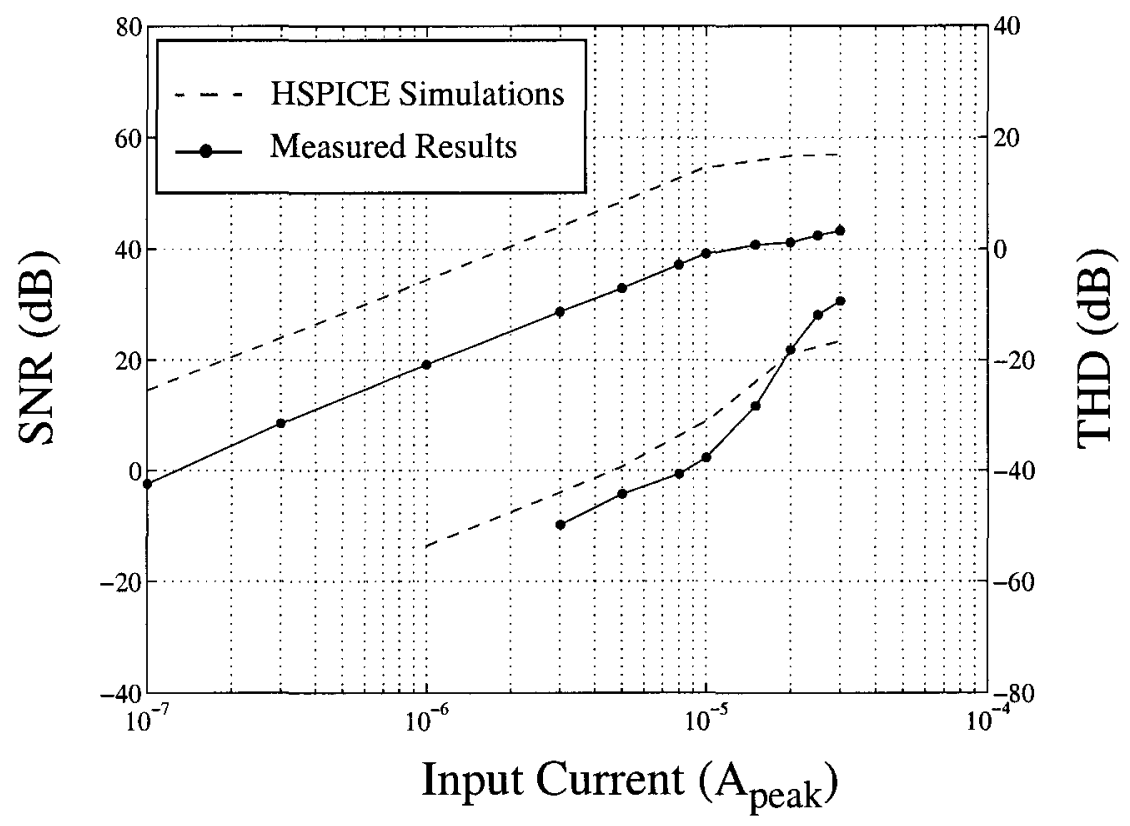

Figure 3.14: SNR (top curves) and THD (bottom curves) for $I_{0}=10 \mu \mathrm{A}$

In measuring both SNR and output noise current, a damping capacitance of $100 \mathrm{pF}$ was used, and the bandwidth was chosen based on the theoretical 3-dB frequency of the circuit. For bias currents of $\mathrm{I}_{\mathrm{o}}=10 \mu \mathrm{A}$ and $50 \mu \mathrm{A}$, the measurement bandwidths were set to $640 \mathrm{kHz}$ and $3.2 \mathrm{MHz}$ respectively, each exceeding the experimentally measured bandwidths by more than $100 \mathrm{kHz}$. The input signal was used for measuring SNR was set to $100 \mathrm{kHz}$, as was the case for THD measurements.

From the measurement of SNR and the harmonic distortion, a third important parameter, the dynamic range can be determined. The dynamic range has been defined as the entire range over which the signal to noise and distortion ratio (SNDR) is greater than unity. The lower end of this range is shown in the figure as the point at which SNR is equal to $0 \mathrm{~dB}$, while the upper end of this range at occurs when the output signal begins to clip, as shown in the figure by the significant increase in THD when $I_{\text {in }}>I_{0}$. The values of dynamic range, SNR, and output noise current measured for the log-domain integrator are each summarized in Table 3.4. 
Table 3.4: Simulated and Measured Noise Performance

\begin{tabular}{|c|c|c|c|}
\hline Parameter & Bias Current & Simulation Results & $\begin{array}{c}\text { Experimental } \\
\text { Results }\end{array}$ \\
\hline \multirow{2}{*}{ Maximum SNR } & $\mathrm{I}_{\mathrm{o}}=10 \mu \mathrm{A}$ & $57.0 \mathrm{~dB}$ & $43.4 \mathrm{~dB}$ \\
\cline { 2 - 4 } & $\mathrm{I}_{\mathrm{o}}=50 \mu \mathrm{A}$ & $60.8 \mathrm{~dB}$ & $44.9 \mathrm{~dB}$ \\
\hline \multirow{2}{*}{$\begin{array}{c}\text { Total Noise } \\
\text { Current }\end{array}$} & $\mathrm{I}_{\mathrm{o}}=10 \mu \mathrm{A}$ & $10.3 \mathrm{nA}_{\mathrm{rms}}$ & $90.0 \mathrm{nA}$ rms \\
\cline { 2 - 4 } & $\mathrm{I}_{\mathrm{o}}=50 \mu \mathrm{A}$ & $37.5 \mathrm{nA}_{\mathrm{rms}}$ & $176 \mathrm{nA}$ rms \\
\hline \multirow{2}{*}{\begin{tabular}{c} 
Dynamic Range \\
\cline { 2 - 4 }
\end{tabular}} & $\mathrm{I}_{\mathrm{o}}=10 \mu \mathrm{A}$ & $59.7 \mathrm{~dB}$ & $39.1 \mathrm{~dB}$ \\
\cline { 2 - 4 } & $\mathrm{I}_{\mathrm{o}}=50 \mu \mathrm{A}$ & $62.5 \mathrm{~dB}$ & $45.6 \mathrm{~dB}$ \\
\hline
\end{tabular}

The maximum experimentally measured values for SNR and dynamic range each exceeded $40 \mathrm{~dB}$. However, these values are approximately $15 \mathrm{~dB}$ lower than predicted by HSPICE simulations, most likely indicating that the experimental test set-up was far from optimal from a noise perspective. All measurements shown here have been made within a Faraday cage, an environment virtually free from electromagnetic interference. However, several possible sources of noise, including the large resistances $(10 \mathrm{k} \Omega-100 \mathrm{k} \Omega)$ used in generating off-chip current references, have likely contributed to the observed degradation. It is likely that the SNR and dynamic range measurements of these filters could be further improved.

An additional note regarding the noise measurements should be made. The circuit board used for measurements, as shown in Figure 3.9(b), was observed to suffer from lowfrequency oscillations. An output spectrum which clearly shows this behaviour is given in Figure 3.15. For this plot, $\mathrm{I}_{\mathrm{o}}=50 \mu \mathrm{A}, \mathrm{I}_{\text {in }}=30 \mu \mathrm{A}_{\text {peak }}$ at $200 \mathrm{kHz}$, and the resolution bandwidth used was $18 \mathrm{~Hz}$. Though the source of the oscillations was not precisely determined, the behaviour was not found to be correlated to the operation of the circuits under test, and as such the oscillations need not be included in the spectral noise measurement. To minimize the impact of these oscillations in the noise calculations, the effective bandwidths used to measure SNR, output noise, and dynamic range were taken starting from $100 \mathrm{kHz}$. Therefore, at a resolution of $10 \mathrm{kHz}$, the first ten frequency 
samples were omitted from calculations. This omission is reasonable, particularly given that upper bandwidth for all measurements exceeded the experimental integrator 3-dB frequency by $100 \mathrm{kHz}$ or more, as mentioned above. The input signal, set to $100 \mathrm{kHz}$ for SNR and dynamic range measurements, was therefore placed at the lower edge of the measurement bandwidth.

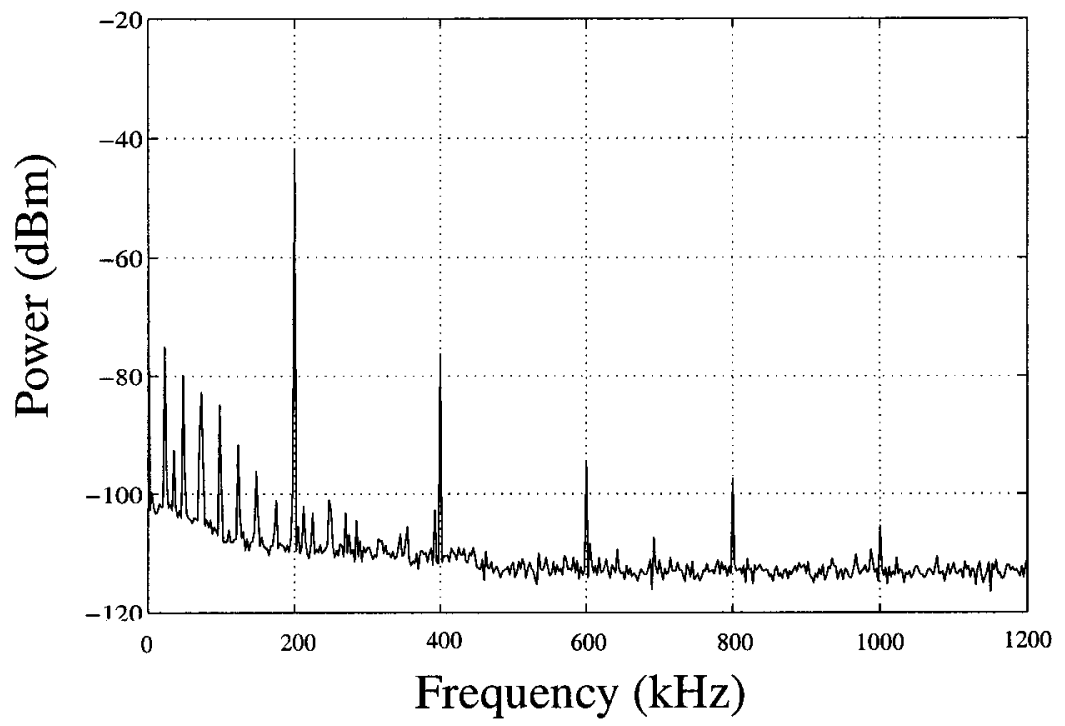

Figure 3.15: Typical output spectrum for $I_{0}=50 \mu \mathrm{A}, I_{\text {in }}=30 \mu A_{\text {peak }}$ at $200 \mathrm{kHz}$

Overall, the maximum SNR and the dynamic range of the integrator were measured to be above $40 \mathrm{~dB}$ and the integrator output noise currents were measured to be between $45 \mathrm{nA}_{\mathrm{rms}}$ to $88 \mathrm{nA}_{\mathrm{rms}}$. This measured performance can likely be further increased through improvements to the experimental test set-up.

\subsection{5 - Summary of the Damped Integrator Performance}

A summary of the damped integrator performance is given in Table 3.5. The measured characteristics demonstrate that the integrator is capable of operation at $10 \mathrm{MHz}$ and above, that the total harmonic distortion (just before the onset of clipping) has been measured at over $-40 \mathrm{~dB}$ for moderate levels of bias current, and that an SNR and dynamic range of over $40 \mathrm{~dB}$ can be achieved. Agreement between the simulated and experimentally measured results is reasonable, indicating that the model developed in Chapter 2 can be a useful tool in the design of bipolar circuity in CMOS technology. 
Table 3.5: A summary of the damped integrator performance

\begin{tabular}{|c|c|c|c|c|}
\hline Parameter & Bias Current & Conditions & $\begin{array}{l}\text { Simulation } \\
\text { Results }\end{array}$ & $\begin{array}{l}\text { Experimental } \\
\text { Results }\end{array}$ \\
\hline \multirow{2}{*}{$\begin{array}{c}\text { Frequency Range } \\
\left(\begin{array}{l}\left(\mathbf{f}_{3 \mathrm{~dB}}\right)\end{array}\right.\end{array}$} & $\mathrm{I}_{\mathrm{o}}=1 \mu \mathrm{A}-100 \mu \mathrm{A}$ & $\mathrm{C}=10 \mathrm{pF}$ & $400 \mathrm{kHz}-38 \mathrm{MHz}$ & $530 \mathrm{kHz}-15 \mathrm{MHz}$ \\
\hline & $\mathrm{I}_{\mathrm{o}}=100 \mu \mathrm{A}$ & $\mathrm{C}=10 \mathrm{pF}-1 \mathrm{nF}$ & $400 \mathrm{kHz}-38 \mathrm{MHz}$ & $220 \mathrm{kHz}-15 \mathrm{MHz}$ \\
\hline \multirow{2}{*}{$\begin{array}{c}\text { THD } \\
\left(\mathrm{I}_{\mathrm{in}}=0.5 \mathrm{I}_{\mathrm{o}}\right)\end{array}$} & $\mathrm{I}_{\mathrm{o}}=10 \mu \mathrm{A}$ & \multirow{2}{*}{$\begin{array}{c}100 \mathrm{kHz} \text { Input } \\
\text { Signal }\end{array}$} & $-39.4 \mathrm{~dB}$ & $-44.3 \mathrm{~dB}$ \\
\hline & $\mathrm{I}_{\mathrm{o}}=50 \mu \mathrm{A}$ & & $-32.0 \mathrm{~dB}$ & $-30.1 \mathrm{~dB}$ \\
\hline \multirow{2}{*}{$\begin{array}{l}\text { Third Order } \\
\text { Intercept } \\
\text { (OIP3) }\end{array}$} & $\mathrm{I}_{\mathrm{o}}=10 \mu \mathrm{A}$ & \multirow{2}{*}{$\begin{array}{l}100 \mathrm{kHz} \text { Input } \\
\text { Signal }\end{array}$} & $180 \mu \mathrm{A}_{\text {peak }}$ & $203 \mu A_{\text {peak }}$ \\
\hline & $\mathrm{I}_{\mathrm{o}}=50 \mu \mathrm{A}$ & & $670 \mu \mathrm{A}_{\text {peak }}$ & $439 \mu A_{\text {peak }}$ \\
\hline \multirow{2}{*}{ Maximum SNR } & $\mathrm{I}_{\mathrm{o}}=10 \mu \mathrm{A}$ & $\mathrm{BW}=640 \mathrm{kHz}$ & $57.0 \mathrm{~dB}$ & $43.4 \mathrm{~dB}$ \\
\hline & $\mathrm{I}_{\mathrm{o}}=50 \mu \mathrm{A}$ & $\mathrm{BW}=3.2 \mathrm{MHz}$ & $60.8 \mathrm{~dB}$ & $44.9 \mathrm{~dB}$ \\
\hline \multirow{2}{*}{$\begin{array}{l}\text { Total Noise } \\
\text { Current }\end{array}$} & $\mathrm{I}_{\mathrm{o}}=10 \mu \mathrm{A}$ & $\mathrm{BW}=640 \mathrm{kHz}$ & $10.3 \mathrm{nA}_{\mathrm{rms}}$ & $90.0 \mathrm{nA}_{\mathrm{rms}}$ \\
\hline & $\mathrm{I}_{\mathrm{o}}=50 \mu \mathrm{A}$ & $\mathrm{BW}=3.2 \mathrm{MHz}$ & $37.5 \mathrm{nA}_{\mathrm{rms}}$ & $176 \mathrm{nA}_{\mathrm{rms}}$ \\
\hline \multirow{2}{*}{ Dynamic Range } & $\mathrm{I}_{\mathrm{o}}=10 \mu \mathrm{A}$ & $\mathrm{BW}=640 \mathrm{kHz}$ & $59.7 \mathrm{~dB}$ & $39.1 \mathrm{~dB}$ \\
\hline & $\mathrm{I}_{\mathrm{o}}=50 \mu \mathrm{A}$ & $\mathrm{BW}=3.2 \mathrm{MHz}$ & $62.5 \mathrm{~dB}$ & $45.6 \mathrm{~dB}$ \\
\hline \multirow{2}{*}{ Power * } & $\mathrm{I}_{\mathrm{o}}=10 \mu \mathrm{A}$ & & $229 \mu \mathrm{W}$ & - \\
\hline & $\mathrm{I}_{\mathrm{o}}=100 \mu \mathrm{A}$ & & $1.47 \mathrm{~mW}$ & - \\
\hline
\end{tabular}

* Power listed above includes input $\mathrm{V} / \mathrm{I}$ and output $\mathrm{I} / \mathrm{V}$ conversion circuitry

The characteristics of this log-domain integrator compare well against subthreshold CMOS integrator implementations, and a comparison between filters designed using this integrator and other CMOS log-domain filters in the literature will be provided at the conclusion of Chapter 4 . Before presenting this comparison, however, a description of a biquadratic and a third order log-domain filter will be provided. The implementation of higher order filters, based upon the CMOS log-domain integrator described above, will be the subject of the next chapter. 


\section{Chapter 4 - The Design of Bipolar Log- Domain Filters in CMOS} Technology

In the previous chapters, it has been established that a lateral PNP transistor with promising characteristics can be fabricated in $0.35 \mu$ CMOS technology and that a logdomain integrator capable of operation at $10 \mathrm{MHz}$ and above can be implemented using these transistors. Having performed these device-level and circuit-level investigations, attention can now be turned to a system-level implementation of log-domain filter circuits in CMOS technology.

The outline of this chapter is as follows: the first section will present the necessary theoretical background to synthesize second and higher-order filters based upon on the log-domain integrator. The second section will provide an overview of the design of a second order low pass biquadratic filter and a third order low pass elliptic filter. The next section will describe in detail the experimentally measured characteristics of the biquadratic and elliptic filters, focusing on frequency response, circuit linearity, and noise performance. The final section will compare the characteristics of the CMOS log-domain filters developed in this chapter to other log-domain filter implementations described in the literature. The comparison will demonstrate that the performance of these $0.35 \mu$ 
CMOS log-domain filters is on par with the performance which could be achieved in a semicustom bipolar process, and will also demonstrate that these $0.35 \mu$ CMOS logdomain filters compare well with other CMOS log-domain filter implementations.

\section{1 - Fundamentals of Log-Domain Filter Design}

The following section will provide the background required to synthesize highorder log-domain filters. First, a design technique known as the operational simulation of LC ladders will be introduced. Using this technique, a signal flow graph representations of LC ladder filters can be generated, and it will be shown that the log-domain integrator of Chapter 3 can used with this representation. The design technique will be used in Section 4.2 to generate biquadratic and third order elliptic log-domain filters. Further information on the methods presented in this section can be found in [1], [6].

\subsection{1 - An Overview of the Operational Simulation of LC Ladders}

The operational simulation of LC ladders is a common technique for synthesizing high order active filters. The objective of this design technique is to develop a circuit which simulates each of the voltages and currents that define the behaviour of an LC (inductor-capacitor) ladder circuit. The generation of LC ladder filters is a well understood and well documented process, and by using LC ladders as prototype filters, many of the desirable characteristics of these filters, notably their low sensitivity to component variation, can be retained in an active circuit implementation (in this case, a log-domain implementation). An overview of the technique is provided below.

A general third-order low-pass LC-ladder filter is shown in Figure 4.1(a). The first step in the synthesis procedure is the development of a system of equations which completely describe the operation of the LC ladder prototype. This can be achieved by writing expressions for the voltage across each capacitor, the current in each inductor, and then applying the Kirchoff's equations to each loop and node in the LC ladder circuit. For the prototype LC shown in Figure 4.1(a), these equations can be expressed as 


$$
\begin{gathered}
v_{1}=\frac{1}{c_{1}} \cdot \int\left(\frac{v_{i}-v_{1}}{r_{S}}-i_{2}\right) d t, \\
i_{2}=\frac{1}{l_{2}} \cdot \int\left(v_{1}-v_{3}\right) d t \\
v_{3}=\frac{1}{c_{3}} \cdot \int\left(i_{2}-v_{3}\right) d t .
\end{gathered}
$$

and

Once a system of equation has been determined, a signal flow graph representation of the filter can be constructed. For the third order low pass filter, the signal flow graph is shown in Figure 4.1(b). Note that the behaviour of the original LC prototype has now been completely described in terms of integration blocks and summing nodes. These two types of components can be realized by a variety of methods which include active-circuit implementations. Such an implementation is shown in Figure 4.1(c). (In this case, $r_{S}$ and $r_{L}$ have been set to unity for simplicity.) Of obvious interest here is the fact that the circuit in Figure 4.1(c) can be realized using the log-domain integrator described in the previous chapter.

Once a circuit representation for the LC ladder has been created, the variables from the prototype filter can be mapped to the final circuit implementation. In this case, there is a direct correspondence between nodes $v_{1}, i_{2}$, and $v_{3}$ in Figure 4.1(b) and nodes $v_{1}, v_{2}$, and $V_{3}$ in Figure 4.1(c), and therefore a relationship can be derived between $c_{1}, 1_{2}$, and $c_{3}$ in the prototype and $\mathrm{C}_{1}, \mathrm{C}_{2}$, and $\mathrm{C}_{3}$ in the final implementation. Once this correspondence is found, the filter design is complete. However, in the case of the synthesis of a logdomain filter, some additional design steps are required in order that the non-linear nature of the log-domain integrator be taken into account. A signal flow graph representation for the log-domain integrator, which could be included in the above analysis, is discussed next. 


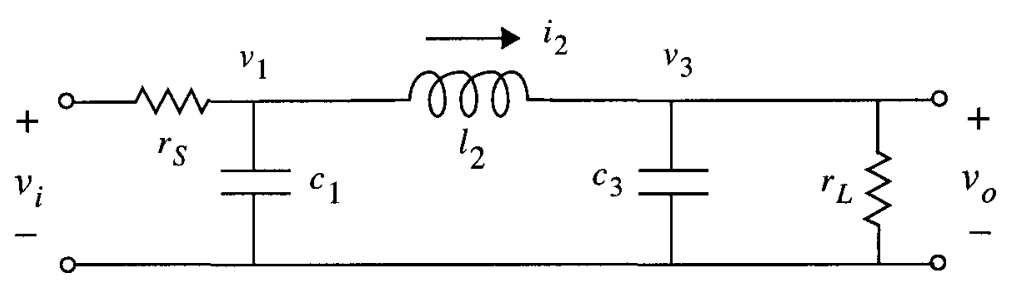

(a)

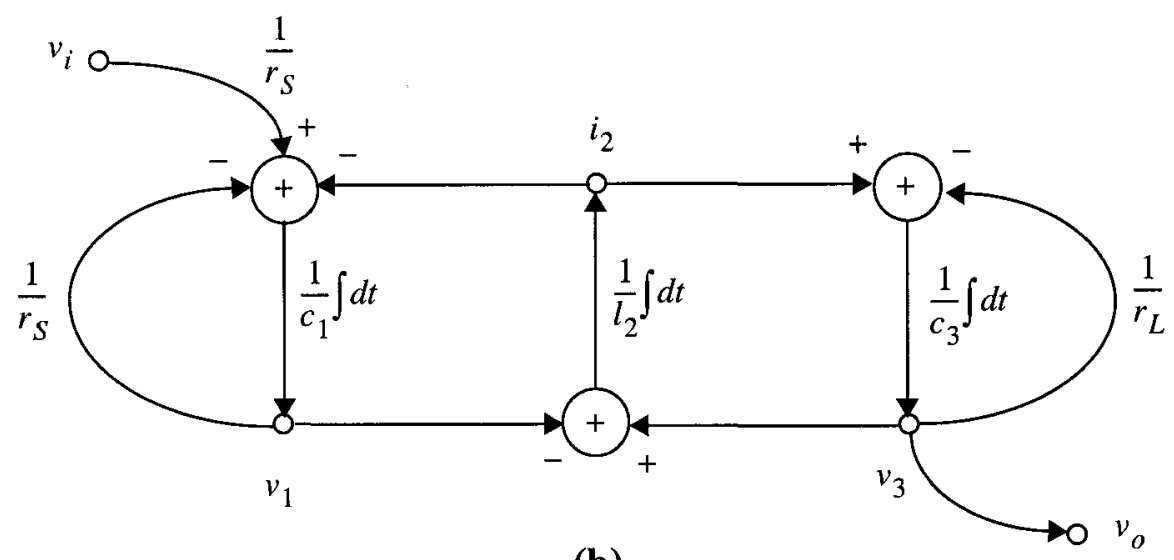

(b)

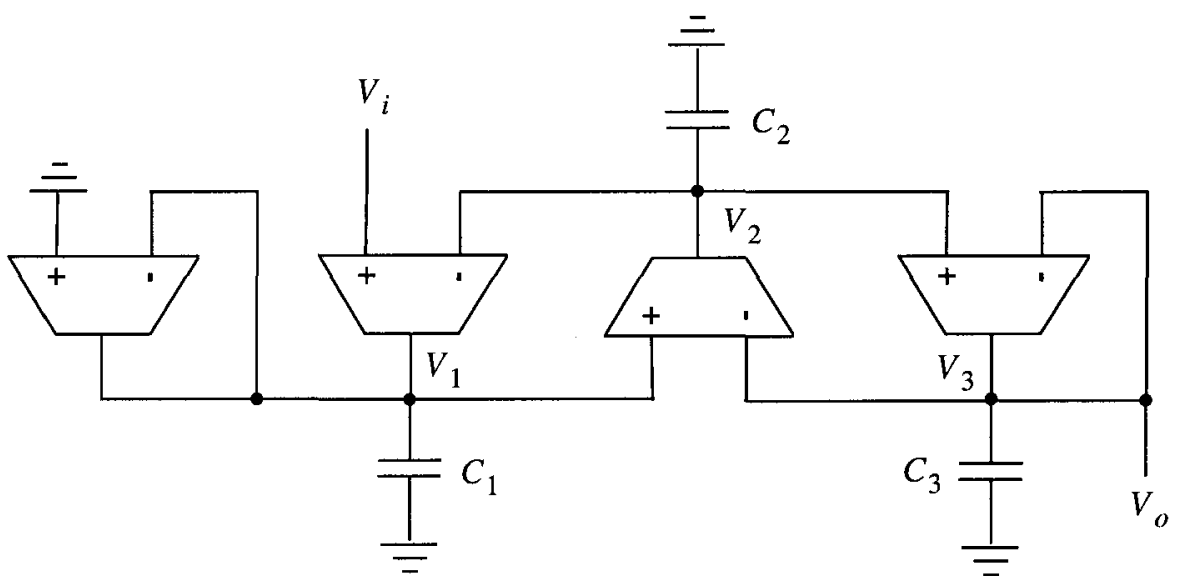

(c)

Figure 4.1: (a) A third order low pass LC ladder filter. (b) The corresponding signal flow graph. (c) An active filter implementation based on operational simulation of original $L C$ ladder. 


\subsection{2 - Operational Simulation for Log-Domain Filters}

The general structure of a log-domain cell, whether it be an integrator or an entire log-domain filter, is shown in Figure 4.2(a). The EXP(x) and LOG(x) operators defined in Eqn. (3.6) and (3.7) have been explicitly shown with symbols in the figure. The diagram is simple, and yet demonstrates an important point: that the input and output signals of this cell are logarithmically compressed, though the function being implemented, denoted by $h(t)$, has a linear representation. Conceptually, the cell can be thought of as performing the operation $\mathrm{h}(\mathrm{t})$ by first exponentially expanding the signal at the cell input, implementing the desired linear function, and then logarithmically compressing the signal at the cell output. A signal flow graph representation for a log-domain integrator which demonstrates this order of operations is shown in Figure 4.2(b). Note that this signal flow graph conforms to the log-domain integrator equation defined in Eqn. (3.13).

To continue with the example of the third order low pass filter of the previous section, the original signal flow graph can now be modified to include the log-domain integrator. This modification is shown in Figure 4.3(a). Observe that a natural cancellation of the majority of $\operatorname{LOG}(x)$ and $\operatorname{EXP}(x)$ operators naturally occurs, simplifying the filter

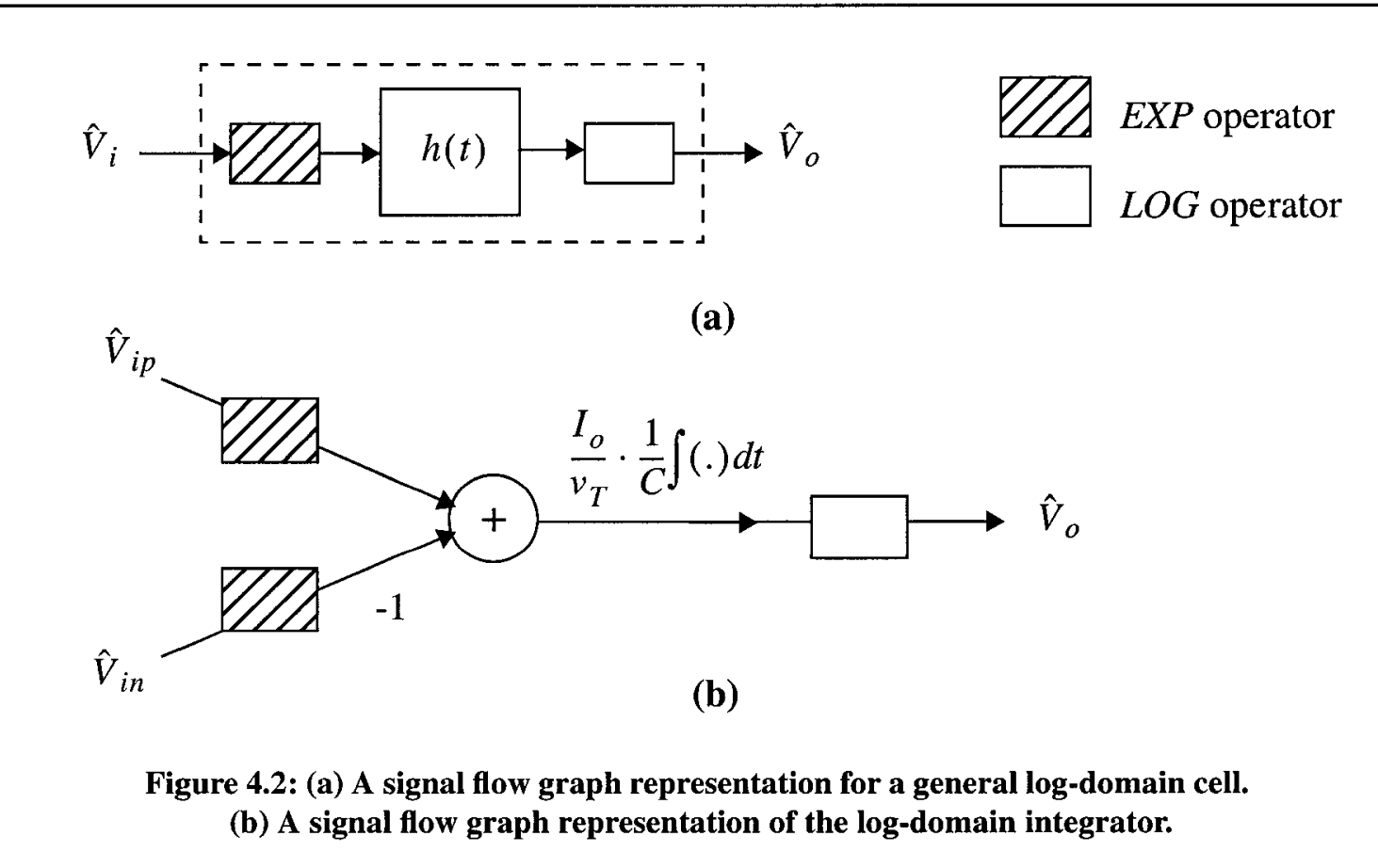


design considerably. Due to cancellation, an entire log-domain filter can in general be implemented using only a single logarithmic compression circuit at the filter input and a single exponential expansion circuit at the filter output, and as shown by the circled LOG(x) and EXP(x) blocks in Figure 4.3(a). A circuit implementation of this signal flow graph is given in Figure 4.3(b).

Once a log-domain circuit representation for the original prototype LC ladder has been created, the variables from the LC prototype filter can be mapped to the final circuit implementation. Finding this correspondence represents the final step in the synthesis of a log-domain filter. The details of mapping the variables from the prototype to the final circuit are best demonstrated by example, as will be provided in the next section.
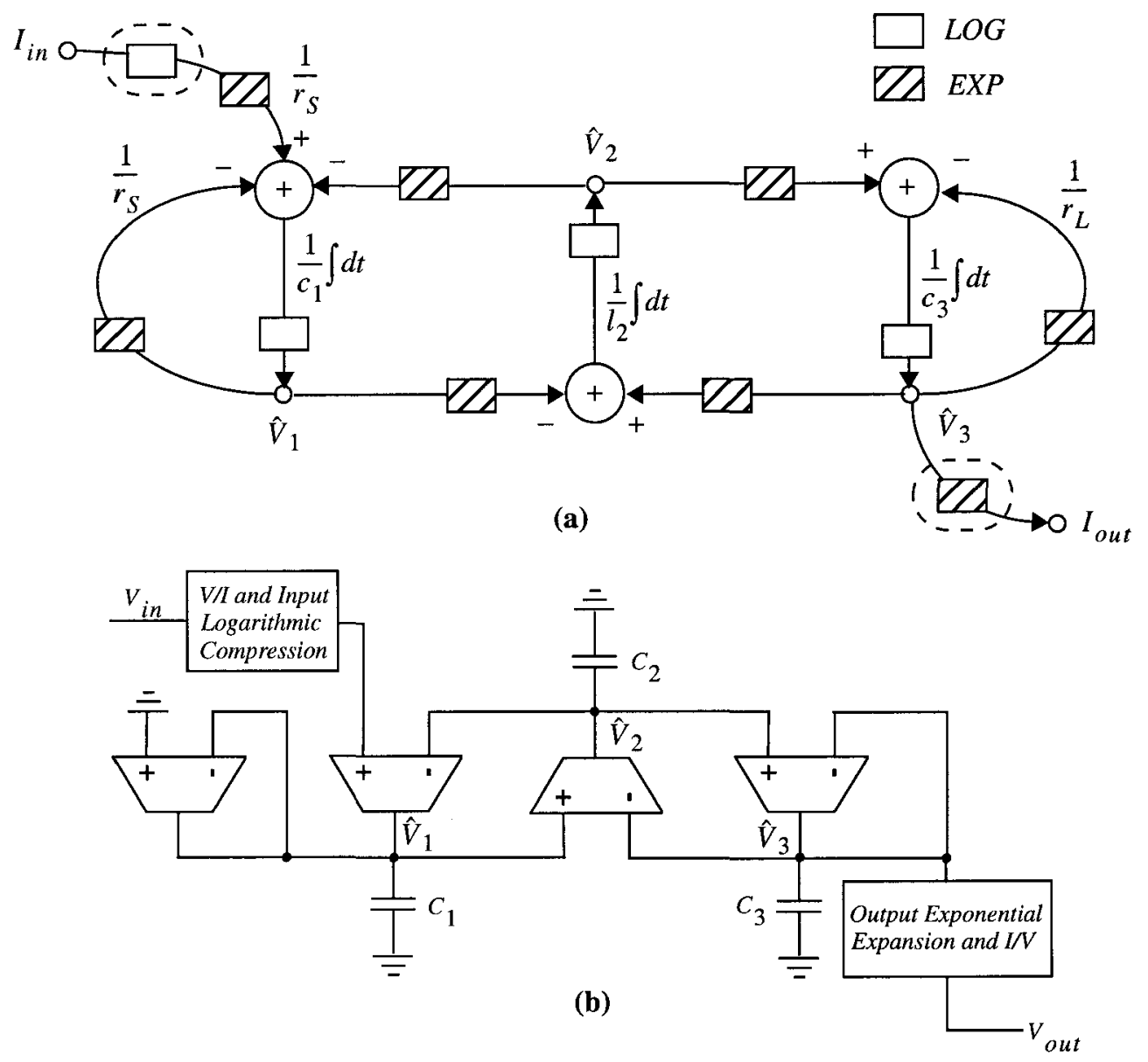

Figure 4.3: (a) A third order low pass filter log-domain signal flow graph.

(b) A log-domain filter implementation of the signal flow graph. 


\section{2 - The Design of Second and Third Order Log-Domain Filters}

The following section will provide an overview of the synthesis of a biquadratic and a third order elliptic filter. Each of the filters designed in this section will be the subject of the experimental tests presented later in this chapter. The material presented here has been adapted from [1], which provides further details on the derivation and synthesis of these filters.

\subsection{1 - A Low Pass Biquadratic Log-Domain Filter}

A low pass biquadratic filter can be designed starting with the LC prototype filter shown in Figure 4.4(a). This circuit has a transfer function which can be expressed as

$$
\frac{V_{o}(s)}{V_{i}(s)}=\frac{1 /(l c)}{s^{2}+s /(r c)+1 /(l c)} .
$$

By comparing this equation with the standard biquadratic form,

$$
H(s)=\frac{k \cdot \omega_{o}^{2}}{s^{2}+\left(\omega_{o} / Q\right) s+\omega_{o}^{2}},
$$

expressions for the natural frequency, $\omega_{o}$, the quality factor, $Q$, and the gain, $k$, of the LC circuit can be determined, and are given by

$$
\omega_{o}=\frac{1}{\sqrt{l c}}, \quad Q=r \cdot \sqrt{\frac{c}{l}}, \quad k=1 .
$$

To simplify matters, the resistance, $r$, will be set to unity for the analysis which follows.

The set of equations which completely define the operation of the biquadratic prototype filter can be expressed as

$$
I_{1}=\frac{1}{l} \cdot \int\left(V_{i}-V_{2}\right) d t
$$

and

$$
V_{2}=\frac{1}{c} \cdot \int\left(I_{1}-V_{2}\right) d t
$$


A signal flow graph can be generated from these equations, as shown in Figure 4.4(b). By adding LOG(x) and $\operatorname{EXP}(\mathrm{x})$ cells to the diagram, and by mapping the variables names $V_{i}$, $I_{1}$ and $V_{2}$ to $I_{i n}, \hat{V}_{1}$ and $\hat{V}_{2}$, a corresponding log-domain signal flow graph can be generated, as shown in Figure 4.4(c). In this case, the set of equations can be rewritten as

$$
\operatorname{EXP}\left(\hat{V}_{1}\right)=\frac{1}{l} \cdot \int\left\{I_{\text {in }}-\operatorname{EXP}\left(\hat{V}_{2}\right)\right\} d t
$$

and

$$
\operatorname{EXP}\left(\hat{V}_{2}\right)=\frac{1}{c} \cdot \int\left\{\operatorname{EXP}\left(\hat{V}_{1}\right)-\operatorname{EXP}\left(\hat{V}_{2}\right)\right\} d t .
$$

A log-domain circuit implementation of the signal flow graph is shown in Figure 4.4(d). Note that the input compression stage has been combined with the first integrator and is implemented in a manner similar to that shown previously in Figure 3.8(b) for the damped integrator. The circuit equations at nodes $\hat{V}_{1}$ and $\hat{V}_{2}$ can be expressed as

$$
\operatorname{EXP}\left(\hat{V}_{1}\right)=\frac{I_{o}}{v_{T}} \cdot \frac{1}{C_{1}} \cdot \int\left\{I_{\text {in }}-\operatorname{EXP}\left(\hat{V}_{2}\right)\right\} d t
$$

and

$$
\operatorname{EXP}\left(\hat{V}_{2}\right)=\frac{I_{o}}{v_{T}} \cdot \frac{1}{C_{2}} \cdot \int\left\{\operatorname{EXP}\left(\hat{V}_{1}\right)-\operatorname{EXP}\left(\hat{V}_{2}\right)\right\} d t
$$

From a comparison of Eqns. (4.9) to (4.12), the relationship between $l, c, C_{1}$, and $C_{2}$ can be expressed as

$$
C_{1}=\frac{I_{o}}{v_{T}} \cdot l, \quad C_{2}=\frac{I_{o}}{v_{T}} \cdot c
$$

Finally, combining these equations with those listed in Eqn. (4.6), the natural frequency, quality factor and gain of the filter of the log-domain filter can be determined to be

$$
\omega_{o}=\frac{I_{o}}{v_{T}} \cdot \frac{1}{\sqrt{C_{1} C_{2}}}, \quad Q=\sqrt{\frac{C_{2}}{C_{1}}}, \quad k=1 .
$$

By selecting $I_{o}, C_{1}$, and $C_{2}$, the design of the log-domain filter is complete. A simplified circuit schematic for the complete biquadratic filter is shown in Figure 4.5. 


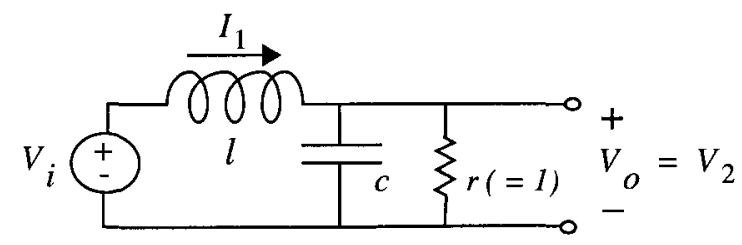

(a)

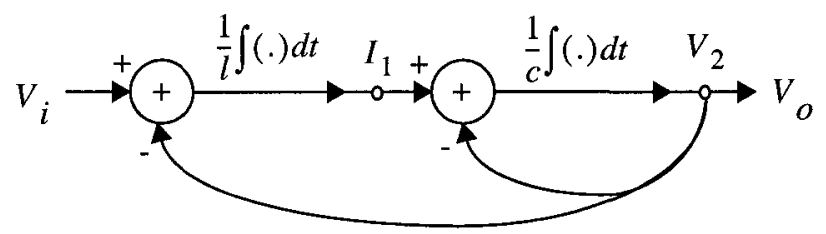

(b)

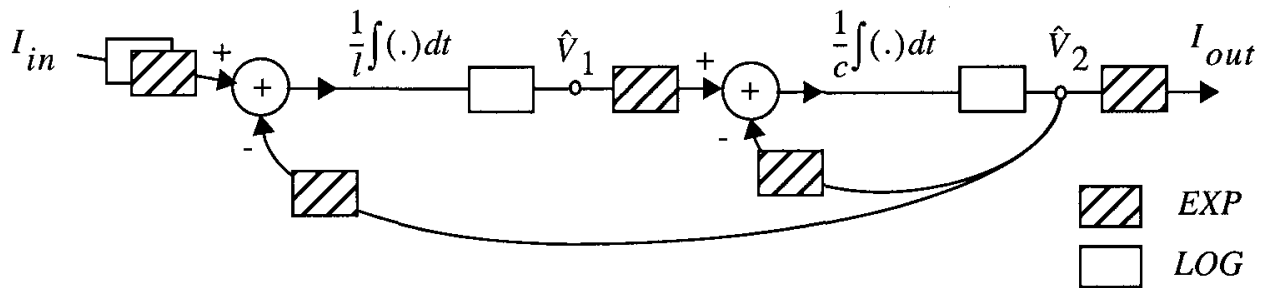

(c)

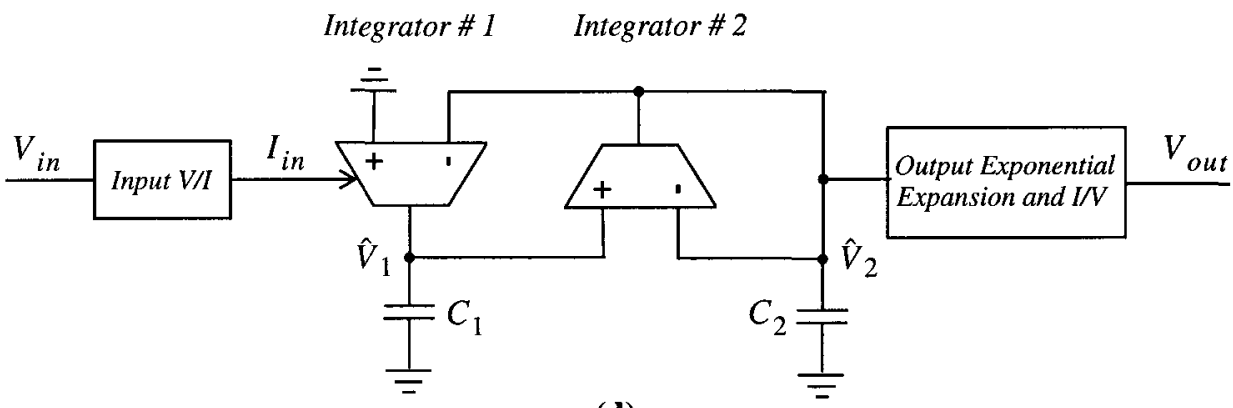

(d)

Figure 4.4: (a) The prototype biquadratic LC filter. (b) The prototype signal flow graph. (c) The log-domain signal flow graph. (d) The log-domain circuit implementation. 


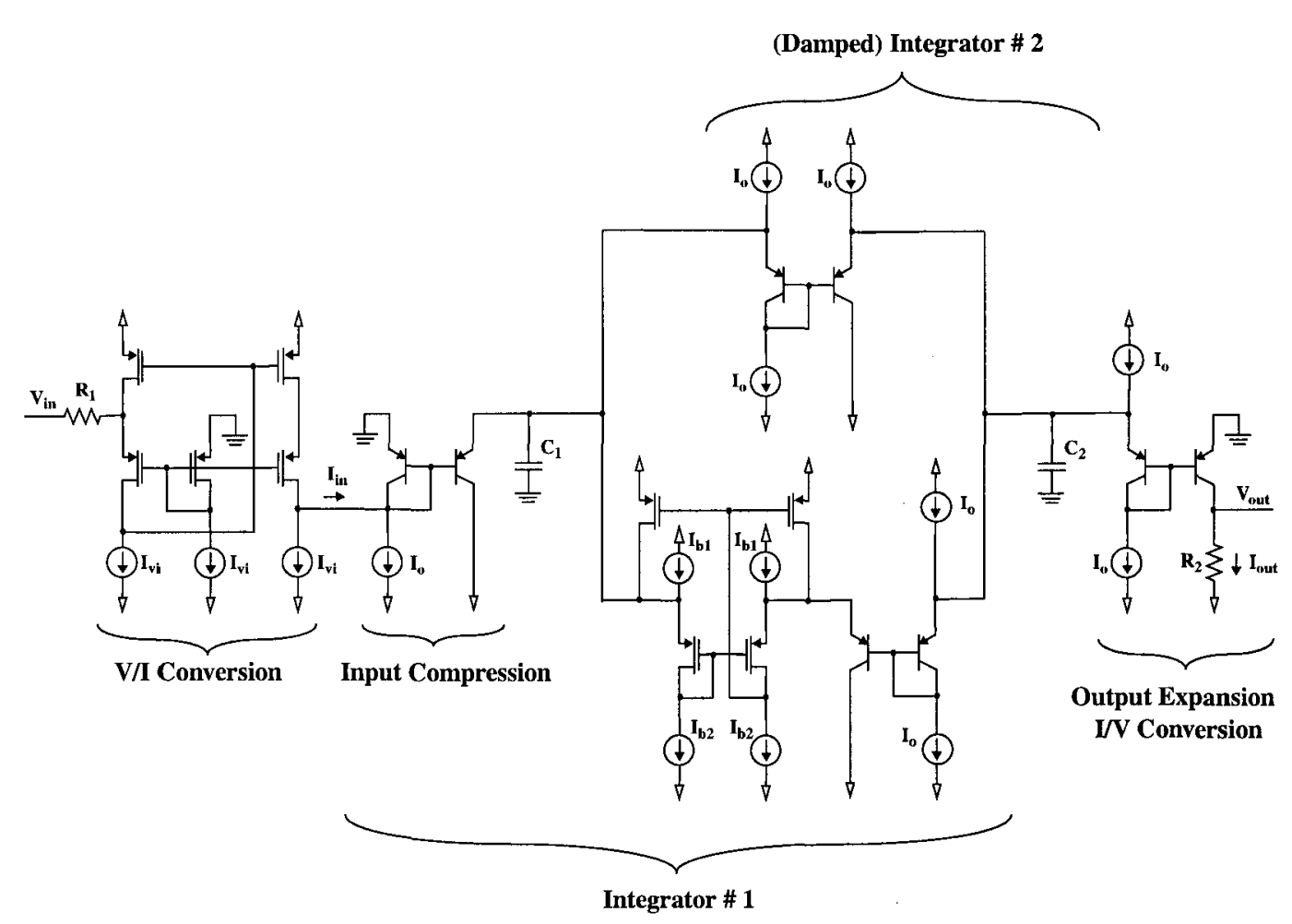

Figure 4.5: A simplified schematic of the final biquadratic filter implementation.

\subsection{2 - A Low Pass Third Order Elliptic Log-Domain Filter}

A low pass third order elliptic filter can be designed starting with the LC ladder prototype filter shown in Figure 4.6(a). Once again, to simplify the analysis, the values of the input and output resistance in the prototype have been set to unity. The set of equations which completely define the operation of the filter can be expressed as

$$
\begin{gathered}
V_{1}=\frac{1}{c_{1}} \cdot \int\left\{V_{i}-V_{1}-I_{2}-c_{4} \frac{d}{d t}\left(V_{1}-V_{3}\right)\right\} d t, \\
I_{2}=\frac{1}{l_{2}} \cdot \int\left\{V_{1}-V_{3}\right\} d t, \\
V_{3}=\frac{1}{c_{3}} \cdot \int\left\{I_{2}-V_{3}+c_{4} \frac{d}{d t}\left(V_{1}-V_{3}\right)\right\} d t .
\end{gathered}
$$

and 
These equations can be manipulated in order to simplify the derivative terms, resulting in

$$
\begin{gathered}
V_{1}=\frac{1}{c_{1}+c_{4}} \cdot \int\left\{V_{i}-V_{1}-I_{2}+c_{4} \frac{d}{d t}\left(V_{3}\right)\right\} d t, \\
I_{2}=\frac{1}{l_{2}} \cdot \int\left\{V_{1}-V_{3}\right\} d t, \\
V_{3}=\frac{1}{c_{3}+c_{4}} \cdot \int\left\{I_{2}-V_{3}+c_{4} \frac{d}{d t}\left(V_{1}\right)\right\} d t .
\end{gathered}
$$

A signal flow graph can be generated from these equations, as shown in Figure 4.6(b). By adding $\operatorname{LOG}(\mathrm{x})$ and $\operatorname{EXP}(\mathrm{x})$ cells to the diagram, and by mapping the variables names $V_{i}$, $V_{1}, I_{2}, V_{3}$ and $\mathrm{V}_{\text {out }}$ to $I_{\text {in }}, \hat{V}_{1}, \hat{V}_{2}, \hat{V}_{3}$, and $I_{\text {out }}$, a corresponding log-domain signal flow graph can be generated, as shown in Figure 4.6(c). In this case, the set of equations can be rewritten as

$$
\begin{gathered}
\operatorname{EXP}\left(\hat{V}_{1}\right)=\frac{1}{c_{1}+c_{4}} \cdot \int\left\{I_{\text {in }}-\operatorname{EXP}\left(\hat{V}_{1}\right)-\operatorname{EXP}\left(\hat{V}_{2}\right)+c_{4} \frac{d}{d t}\left(\hat{V}_{3}\right)\right\} d t \\
\operatorname{EXP}\left(\hat{V}_{2}\right)=\frac{1}{l_{2}} \cdot \int\left\{\operatorname{EXP}\left(\hat{V}_{1}\right)-\operatorname{EXP}\left(\hat{V}_{3}\right)\right\} d t \\
\text { and } \operatorname{EXP}\left(\hat{V}_{3}\right)=\frac{1}{c_{3}+c_{4}} \cdot \int\left\{\operatorname{EXP}\left(\hat{V}_{2}\right)-\operatorname{EXP}\left(\hat{V}_{3}\right)+c_{4} \frac{d}{d t}\left(\hat{V}_{1}\right)\right\} d t
\end{gathered}
$$

Note that the log-domain filter cannot be directly implemented from the above equations, since we do not have a functional block analogous to a log-domain differentiator. However, as is the case in other active filter technologies, the realization of the differentiator can be approximated by placing a single capacitor placed between the $\hat{V}_{1}$ and $\hat{V}_{3}$, nodes of the circuit [1]. (Indeed, a floating capacitor placed between the $V_{1}$ and $\mathrm{V}_{3}$ nodes was used to generate the zero in the transfer function of the original LC ladder prototype.) The corresponding log-domain filter implementation is shown in Figure 4.6(d). 
The circuit equations ${ }^{1}$ generated from this figure are given by

$$
\begin{gathered}
\operatorname{EXP}\left(\hat{V}_{1}\right)=\frac{I_{o}}{{ }^{v_{T}}} \cdot \frac{1}{C_{1}+C_{4}} \cdot \int\left\{I_{i n}-\operatorname{EXP}\left(\hat{V}_{1}\right)-\operatorname{EXP}\left(\hat{V}_{2}\right)+\frac{{ }^{v_{T} C_{4}}}{I_{o}} \cdot \frac{d}{d t}\left(\operatorname{EXP}\left(\hat{V}_{3}\right)\right)\right\} d t, \\
\operatorname{EXP}\left(\hat{V}_{2}\right)=\frac{I_{o}}{v_{T}} \cdot \frac{1}{C_{2}} \cdot \int\left\{\operatorname{EXP}\left(\hat{V}_{1}\right)-\operatorname{EXP}\left(\hat{V}_{3}\right)\right\} d t,
\end{gathered}
$$

and $\operatorname{EXP}\left(\hat{V}_{3}\right)=\frac{I_{o}}{v_{T}} \cdot \frac{1}{C_{3}+C_{4}} \cdot \int\left\{\operatorname{EXP}\left(\hat{V}_{2}\right)-\operatorname{EXP}\left(\hat{V}_{3}\right)+\frac{{ }^{v_{T} C_{4}}}{I_{o}} \cdot \frac{d}{d t}\left(\operatorname{EXP}\left(\hat{V}_{3}\right)\right)\right\} d t$

From a comparison of Eqns (4.21) to (4.26), the relationship between the circuit elements can be expressed as

$$
\left\{C_{1}, C_{2}, C_{3}, C_{4}\right\}=\frac{I_{o}}{v_{T}} \cdot\left\{c_{1}, l_{2}, c_{3}, c_{4}\right\}
$$

As the final step, the values of the capacitors and inductors for the prototype filter would be determined based on the desired filter specifications. For an elliptic filter, these can be read from filter tables [35] or determined with computer-aided design tools. Once these values have been mapped to the log-domain filter implementation according to Eqn. (4.27), the filter design is complete. A simplified circuit schematic for the complete third order elliptic filter is shown in Figure 4.7.

The biquadratic and elliptic filters described above have been fabricated and tested, and an experimental characterization of these filters will be provided in the next section. It will be demonstrated that filters with desired responses can be generated using the synthesis methods presented above.

1. These equations have been generated using an approximation that $e^{\hat{V}_{1} / v_{T}}=e^{\hat{V}_{3} / v_{T}}$, as described in detail in [1], and is the reason why the realization of the differentiator function is only "approximate". 


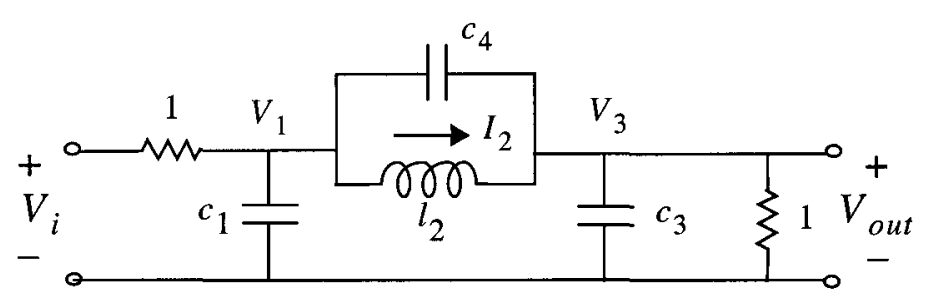

(a)
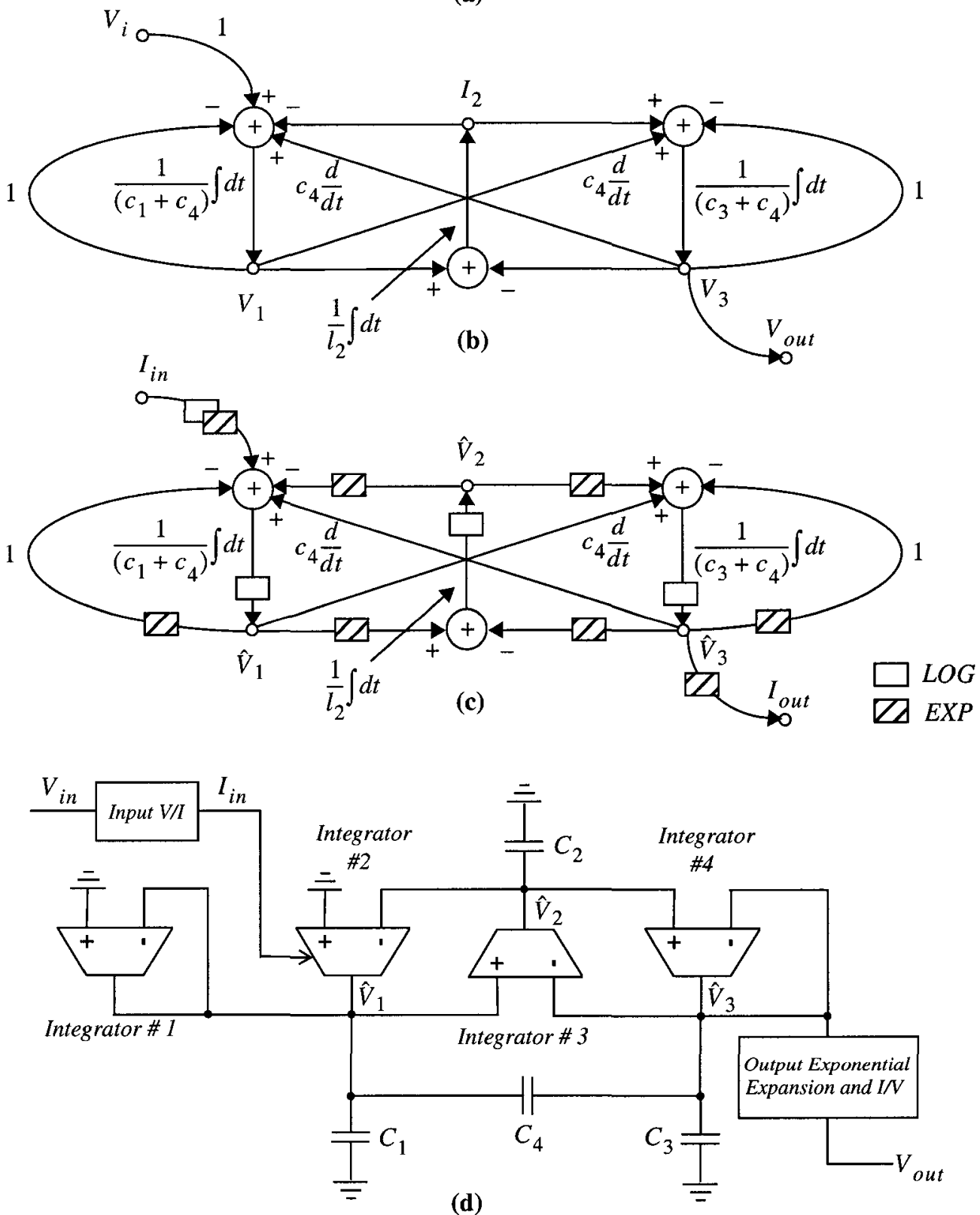

Figure 4.6: (a) The prototype elliptic LC ladder filter (b) The prototype linear signal flow graph (c) The log-domain signal flow graph (d) The log-domain-circuit implementation 


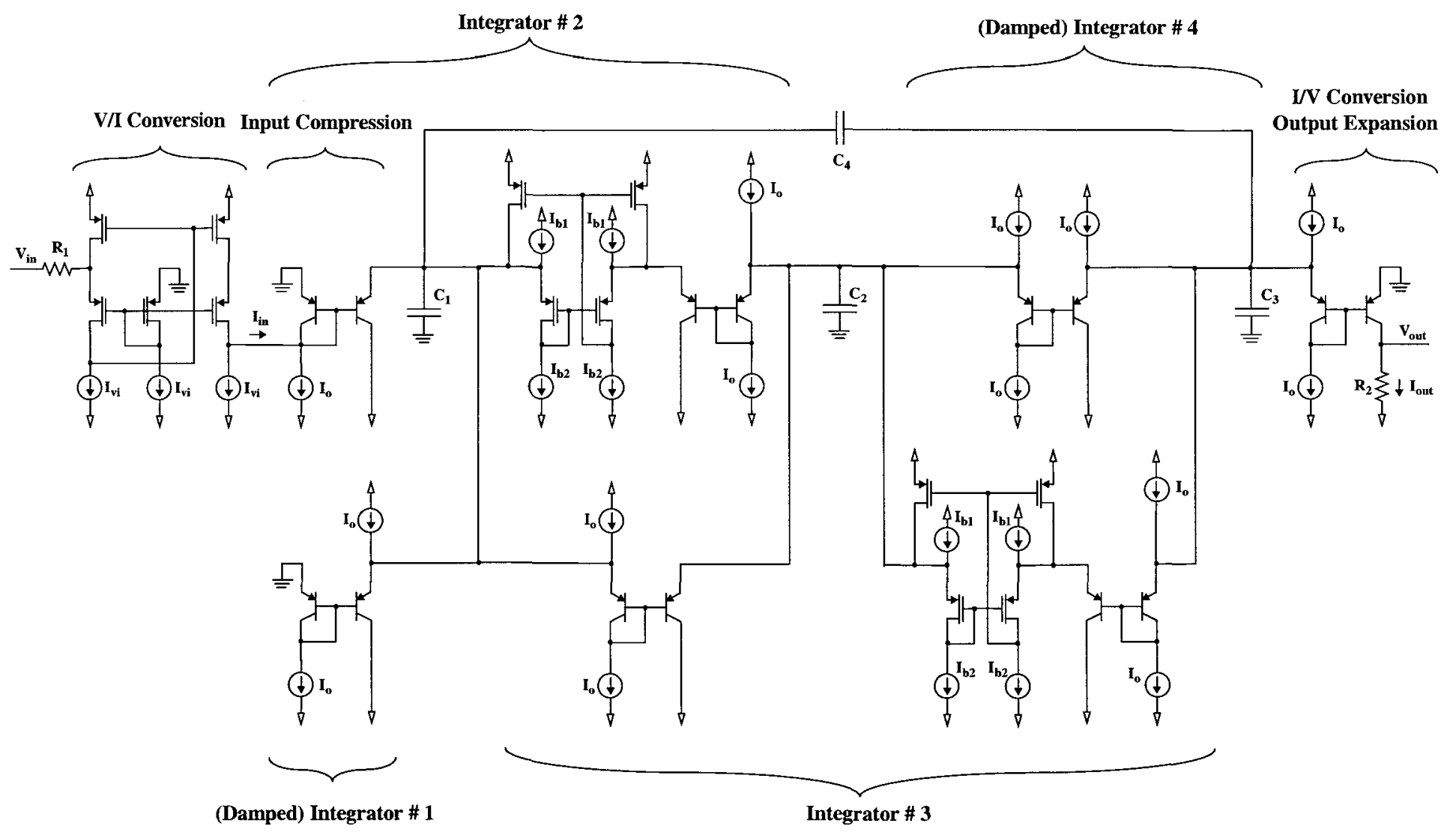

Figure 4.7: A simplified schematic of the final elliptic filter implementation 


\section{3 - Experimental Results}

This section will discuss the experimental performance of biquadratic and elliptic low pass filters fabricated in $0.35 \mu \mathrm{CMOS}$ technology. The three categories of tests have been used to characterize the filter performance: frequency response, linearity, and noise performance. In the final section of this chapter, a comparison between the measured performance versus other filters described in the literature will be provided. First, the circuit under test and the test conditions will be described.

\subsection{1 - Description of the Log-Domain Filter Test Circuits}

The biquadratic and elliptic filters used in testing are shown in Figures 4.5 and 4.7. These circuit diagrams have been simplified for visual clarity, though all measurements have been made using the complete integrator circuitry shown in Figures 3.5 to 3.7.

Several different ranges of capacitor values for both types of filters were used, and are listed in Table 4.1. The theoretical cut-off frequencies (based on ideal transfer functions) for bias currents of $\mathrm{I}_{\mathrm{o}}=100 \mu \mathrm{A}$ have been listed in each case. The biquadratic filters (Filter \#1 - \#3) have been designed to have a constant quality factor of $\sqrt{2}$, and were implemented with external capacitors. The elliptic filters (Filters \#4 - \#6) have each been designed to have a maximum passband ripple of $1 \mathrm{~dB}$ and a stopband attenuation of $30 \mathrm{~dB}$. Filters \#5 and \#6 were implemented with integrated capacitors, while Filter \# 4 was implemented with external capacitors.

The input and output resistors for the biquadratic filter were set to $500 \Omega$, while the input and output resistors for the elliptic filter were set to $500 \Omega$ and $1 \mathrm{k} \Omega$ respectively. The doubled value of output resistance of the elliptic filter was used to compensate for the $6 \mathrm{~dB}$ passband attenuation present in original LC ladder prototype filter.

The values of $I_{0}, I_{b 1}, I_{b 2}, I_{v i}$, and $I_{s h}$ could be independently controlled with offchip references. For current biases from $\mathrm{I}_{\mathrm{o}}=10 \mu \mathrm{A}$ to $100 \mu \mathrm{A}$, the values of $\mathrm{I}_{\mathrm{b} 1}, \mathrm{I}_{\mathrm{b} 2}$ and $\mathrm{I}_{\mathrm{vi}}$ were set to be equal to $\mathrm{I}_{\mathrm{o}}$, but for current biases with $\mathrm{I}_{\mathrm{o}}<10 \mu \mathrm{A}, \mathrm{I}_{\mathrm{b} 1} \mathrm{I}_{\mathrm{b} 2}$, and $\mathrm{I}_{\mathrm{vi}}$ were kept fixed at $10 \mu \mathrm{A}$. A value of $\mathrm{I}_{\mathrm{sh}}=5 \mu \mathrm{A}$ was used in all tests described in this section. 
Table 4.1 - Capacitor values used for biquadratic and elliptic filter testing

\begin{tabular}{|c|c|c|c|}
\hline & Filter Type & Capacitor Values & $\begin{array}{c}\text { Theoretical Cut-Off } \\
\text { Frequency } \\
\left(\mathrm{I}_{\mathrm{o}}=100 \mu \mathrm{A}\right)\end{array}$ \\
\hline Filter \# 1 & \multirow{3}{*}{ Biquadratic } & $\mathrm{C}_{1}=200 \mathrm{pF}, \mathrm{C}_{2}=100 \mathrm{pF}$ & $4.5 \mathrm{MHz}$ \\
\hline Filter \# 2 & & $\mathrm{C}_{1}=100 \mathrm{pF}, \mathrm{C}_{2}=50 \mathrm{pF}$ & $9.0 \mathrm{MHz}$ \\
\hline Filter \# 3 & & $\mathrm{C}_{1}=50 \mathrm{pF}, \mathrm{C}_{2}=25 \mathrm{pF}$ & $18 \mathrm{MHz}$ \\
\hline Filter \# 4 & \multirow{3}{*}{ Elliptic } & $\begin{array}{c}\mathrm{C}_{1}=275 \mathrm{pF}, \mathrm{C}_{2}=125 \mathrm{pF} \\
\mathrm{C}_{3}=275 \mathrm{pF}, \mathrm{C}_{4}=50 \mathrm{pF}\end{array}$ & $4.0 \mathrm{MHz}$ \\
\hline Filter \# 5 & & $\begin{array}{l}\mathrm{C}_{1}=110 \mathrm{pF}, \mathrm{C}_{2}=50 \mathrm{pF} \\
\mathrm{C}_{3}=110 \mathrm{pF}, \mathrm{C}_{4}=20 \mathrm{pF}\end{array}$ & $10 \mathrm{MHz}$ \\
\hline Filter \# 6 & & $\begin{array}{c}\mathrm{C}_{1}=44 \mathrm{pF}, \mathrm{C}_{2}=20 \mathrm{pF} \\
\mathrm{C}_{3}=44 \mathrm{pF}, \mathrm{C}_{4}=8 \mathrm{pF}\end{array}$ & $25 \mathrm{MHz}$ \\
\hline
\end{tabular}

The power supplies used in testing the circuit were $\mathrm{V}_{\mathrm{DD}}=2.5 \mathrm{~V}$, AGND (analog ground) $=2.0 \mathrm{~V}$, and $\mathrm{V}_{\mathrm{SS}}=0 \mathrm{~V}$. The frequency response was once again found to be slightly improved by lowering the $\mathrm{V}_{\mathrm{DD}}$ to between $2.35 \mathrm{~V}$ and $2.45 \mathrm{~V}$, and most measurements were taken from this slightly lowered voltage. The bias voltage of the cascoded transistors within the integrator were kept at $0.55 \mathrm{~V}$ above $\mathrm{V}_{\mathrm{SS}}$ for all tests described here.

The log-domain filters were manufactured in $0.35 \mu$ CMOS technology. A microphotograph of the biquadratic and elliptic filters are shown in Figure 4.8. The total die areas for the biquadratic and elliptic filter (not including current sources) were $340 \mu \mathrm{x}$ $110 \mu$ and $515 \mu \times 110 \mu$ respectively, and the total area for on-chip (poly/poly) capacitors in this technology was $1700 \mu^{2} / \mathrm{pF}$. All measurements were made using a Hewlett Packard 3588A Spectrum Analyzer, a Tektronix TDS800 Oscilloscope, and Hewlett Packard 3314A Function Generator. The circuit boards used for testing were similar to that shown previously in Figure 3.9(b). 


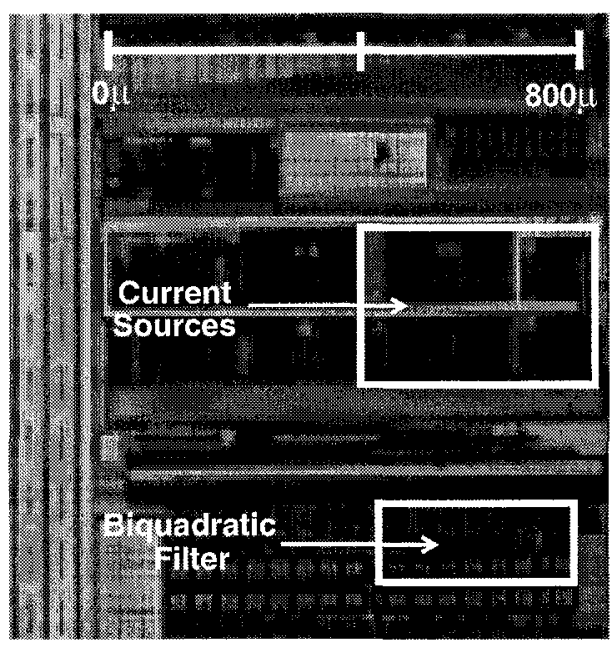

(a)

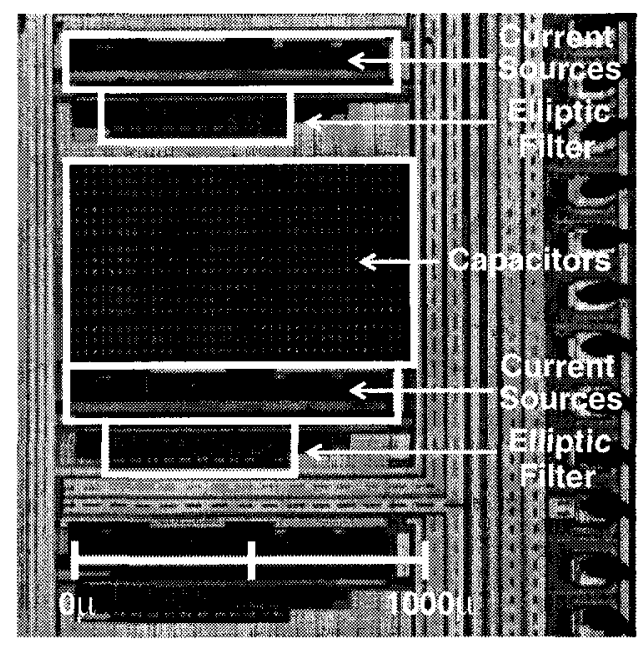

(b)

Figure 4.8: (a) A microphotograph of the biquadratic filter and associated circuitry (b) A microphotograph of the elliptic filter and associated circuitry

\subsection{2 - A Biquadratic Low Pass Filter}

This section will describe the measured performance of a low pass biquadratic logdomain filter, and will begin with an examination of the filter frequency response. The experimentally measured response of the filter is shown in Figures 4.9 and 4.10. In the first figure, the filter capacitance has been held constant while the bias current $I_{0}$ has been varied from $1 \mu \mathrm{A}$ to $100 \mu \mathrm{A}$. In the second figure, a constant current of $\mathrm{I}_{\mathrm{o}}=50 \mu \mathrm{A}$ has been used, while the filter capacitor values have been varied. The simulated response has been included in the figure for comparison.

The frequency response was very well predicted by simulation, the most significant deviation being the small amount of peaking observed when large currents and relatively small capacitances were used for the filter. Though relatively large off-chip capacitors were used for these tests, a maximum 3-dB frequency of $5.0 \mathrm{MHz}$ was observed, and the ability to tune this cut-off frequency of the filter by almost two decades using the bias current, $\mathrm{I}_{\mathrm{o}}$, is clearly demonstrated in Figure 4.9. 


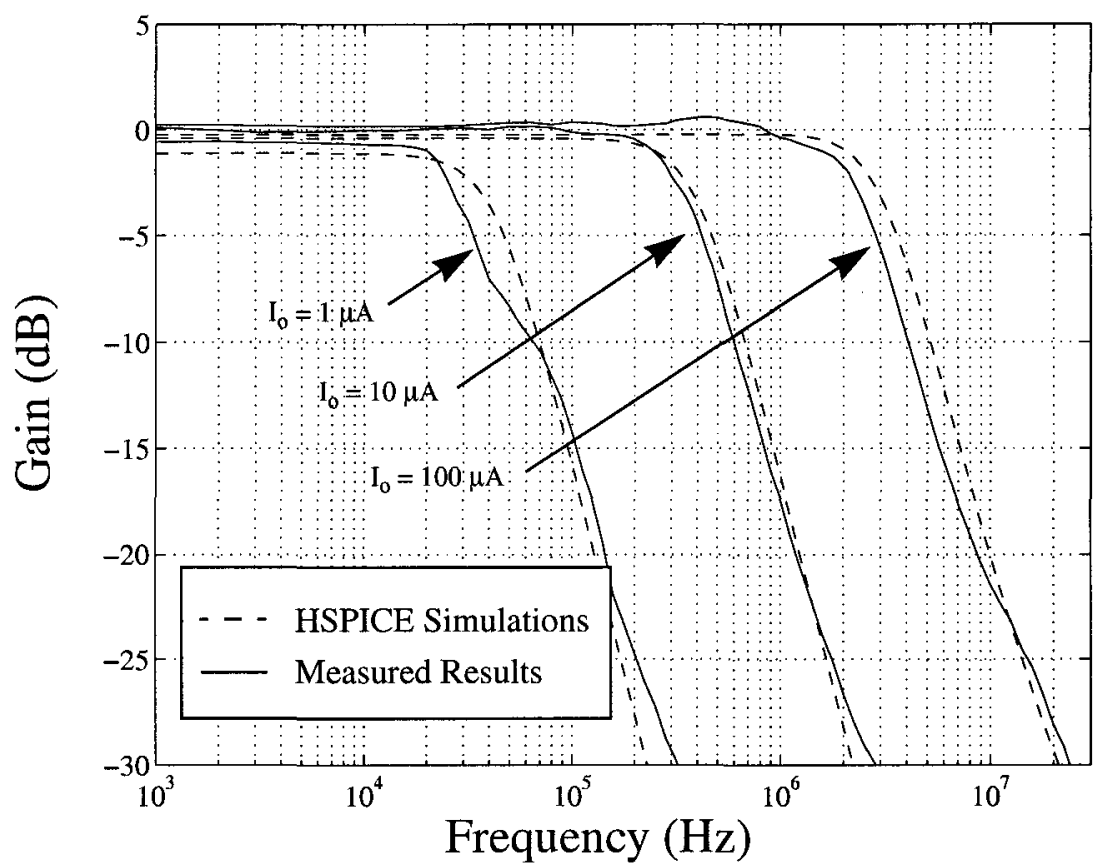

Figure 4.9 Frequency response of biquadratic filter \# $1, I_{0}=1 \mu \mathrm{A}=100 \mu \mathrm{A}$.

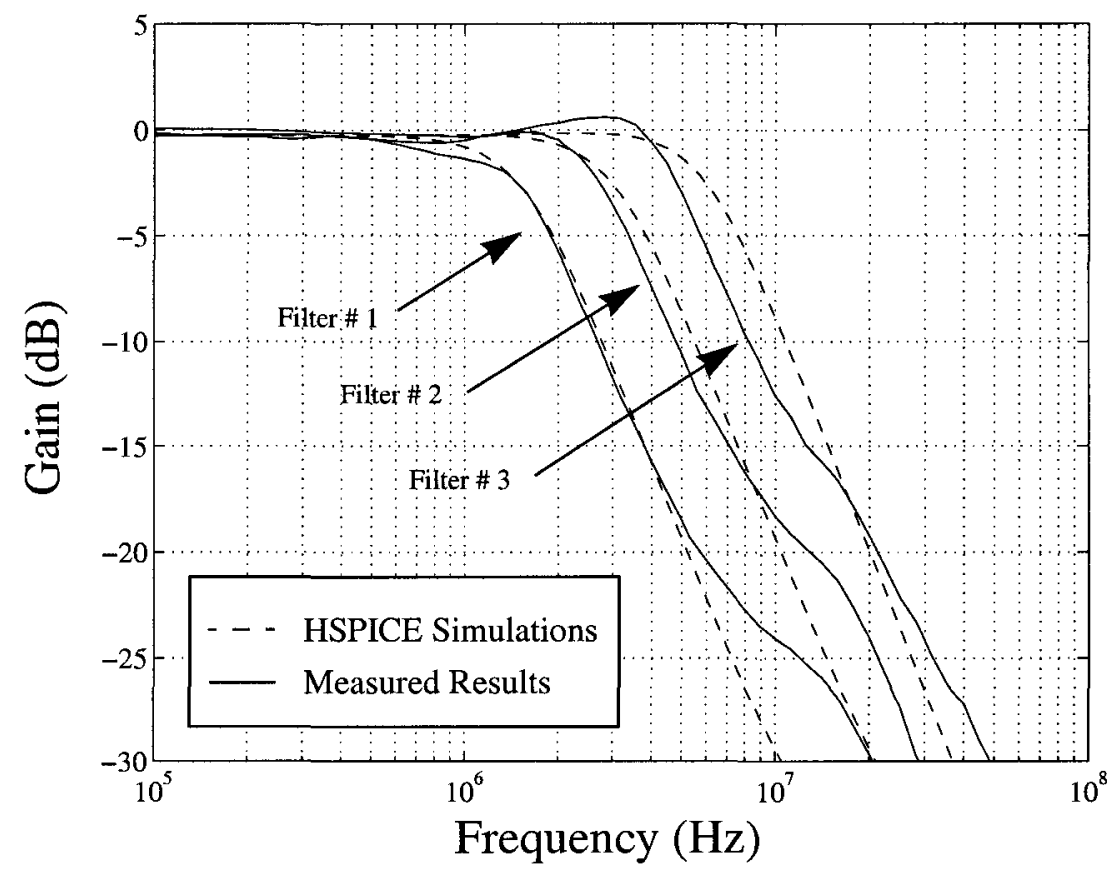

Figure 4.10 Frequency response of biquadratic filter \# 1 - \# 3, $I_{0}=50 \mu \mathrm{A}$ 
The overall linearity of the filter was characterized using measurements of both total harmonic distortion and third order intercept points, and the results of these tests are shown in Figures 4.11 and 4.12. A bias current of $\mathrm{I}_{\mathrm{O}}=10 \mu \mathrm{A}$ and an input signal of 100 $\mathrm{kHz}$ was used in each case. The THD of the filter at $\mathrm{I}_{\mathrm{in}}=0.5 \mathrm{I}_{\mathrm{o}}$ was $-43.1 \mathrm{~dB}$ and the output third order intercept point was measured to be $149 \mu \mathrm{A}_{\text {peak. }}$. Both values are close to, though slightly lower than, the corresponding values measured for the single damped integrator in Chapter 3, a result which reasonable since the filter order and circuit complexity have been increased.

The noise performance of the filter was characterized using measurements of SNR, dynamic range, and output noise current. The SNR of Filter \# 2, with $\mathrm{I}_{\mathrm{o}}$ set to $10 \mu \mathrm{A}$ and with a measurement bandwidth of $800 \mathrm{kHz}$, is shown in Figure 4.11. The maximum value of SNR was $39.6 \mathrm{~dB}$, and the total dynamic range, as defined in Chapter 3, was $35.2 \mathrm{~dB}$. The total output noise current over the measurement bandwidth was $121 \mathrm{nA}_{\mathrm{rms}}$. By increasing the bias current to $50 \mu \mathrm{A}$, both the SNR and dynamic range could be improved by several decibels. However, as in the case of the damped integrator, the measured noiserelated performance is significantly lower than anticipated, suggesting that there may be significant room for improvement in the test and measurement set-up. A summary of all biquadratic filter measurements is given in Table 4.2.

An important note should be made with regard to difficulties which arose from the presence of multiple operating points within these filter circuits. As described in [17], CMOS log-domain filters which contain some form of positive feedback loops within their topologies can suffer from this effect, which can take the form of several circuit nodes settling to unintended states. This behaviour has been observed experimentally in both the biquadratic and elliptic filters described in this work. Specifically, for circuits in which capacitances were placed off-chip (in which internal circuit nodes were directly accessible) the voltages at these capacitive nodes were often observed to settle to undesired voltages. Under such conditions, the filter is inoperable. 


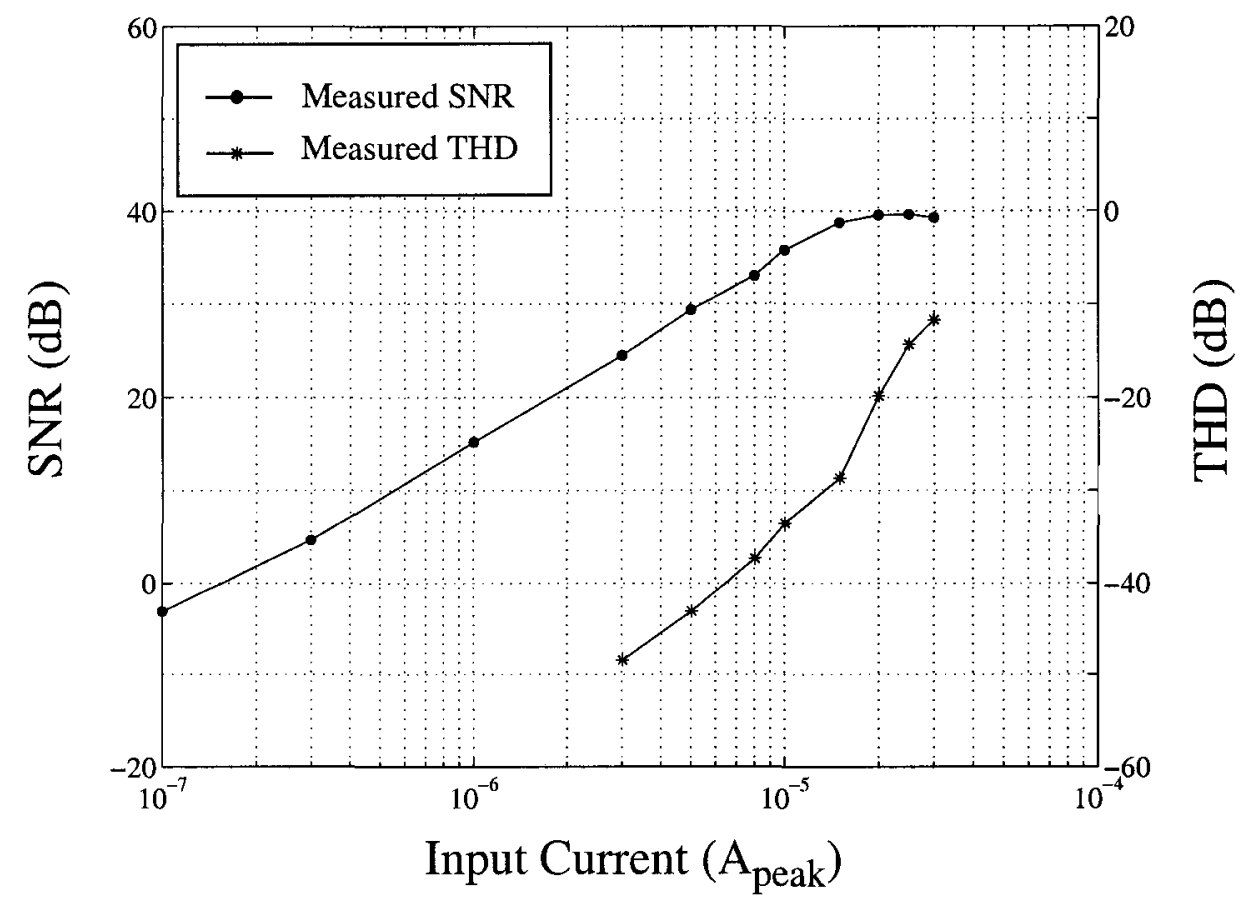

Figure 4.11: SNR and THD for filter \# 2, $I_{0}=10 \mu \mathrm{A}, I_{\text {in }}=100 \mathrm{kHz}, B W=800 \mathrm{kHz}$

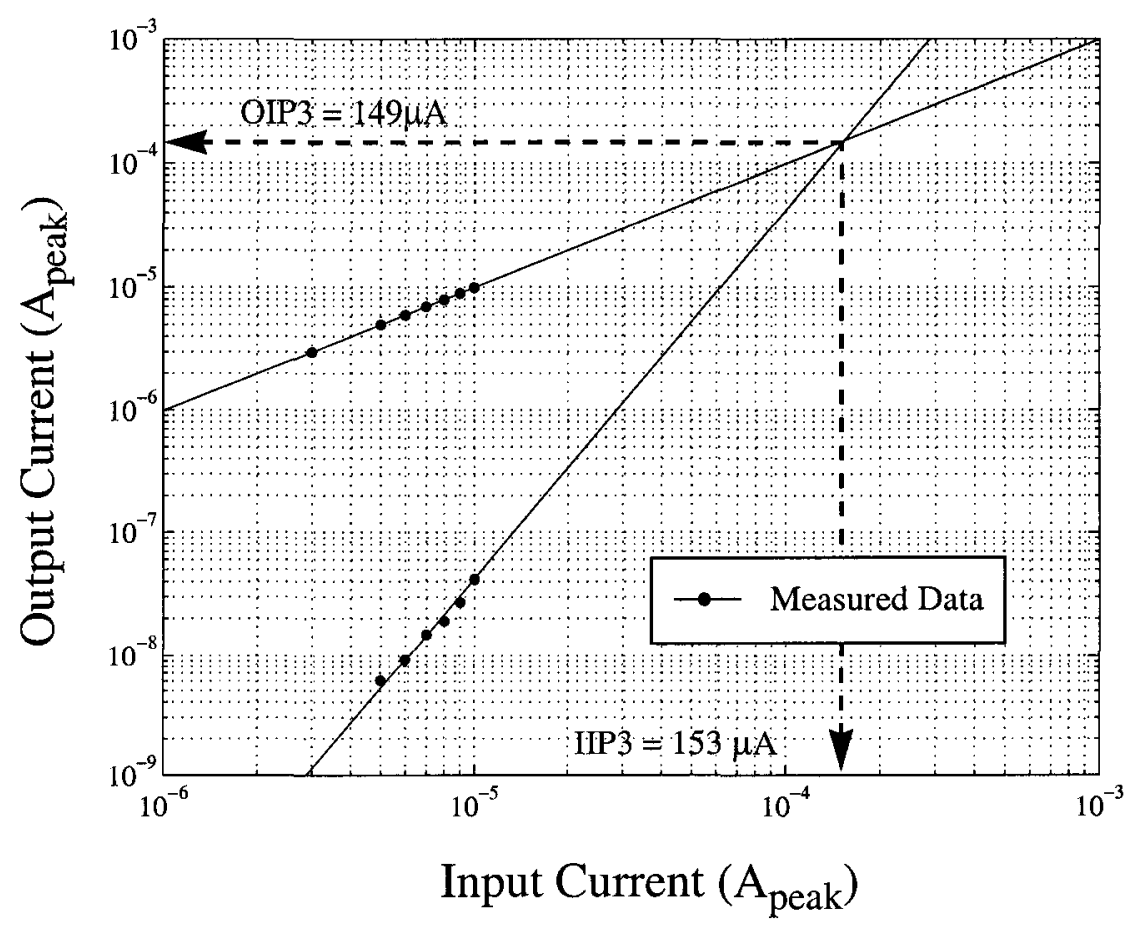

Figure 4.12 Third order intercept points for filter $\# 1, I_{0}=10 \mu \mathrm{A}, I_{\text {in }}=100 \mathrm{kHz}$ 
Table 4.2 - A summary of the biquadratic filter performance

\begin{tabular}{|c|c|c|c|}
\hline Parameter & Bias Current & Conditions & $\begin{array}{c}\text { Experimental } \\
\text { Results }\end{array}$ \\
\hline \multirow{2}{*}{$\begin{array}{l}\text { Frequency Range } \\
\qquad\left(\mathrm{f}_{3 \mathrm{~dB}}\right)\end{array}$} & $\mathrm{I}_{\mathrm{o}}=1 \mu \mathrm{A}-100 \mu \mathrm{A}$ & Filter \#1 & $26 \mathrm{kHz}-2.2 \mathrm{MHz}$ \\
\hline & $\mathrm{I}_{\mathrm{o}}=50 \mu \mathrm{A}$ & Filter \#1 - \#3 & $1.6 \mathrm{MHz}-5.0 \mathrm{MHz}$ \\
\hline THD & $\mathrm{I}_{\mathrm{o}}=10 \mu \mathrm{A}$ & Filter \# $1, \mathrm{I}_{\mathrm{in}}=0.5 \mathrm{I}_{\mathrm{o}}$ at $100 \mathrm{kHz}$ & $-43.1 \mathrm{~dB}$ \\
\hline Third Order Int. & $\mathrm{I}_{\mathrm{o}}=10 \mu \mathrm{A}$ & Filter \# $1, \mathrm{I}_{\mathrm{in}}=100 \mathrm{kHz}$ & $149 \mu \mathrm{A}_{\text {peak }}$ \\
\hline \multirow{2}{*}{ Maximum SNR } & $\mathrm{I}_{\mathrm{o}}=10 \mu \mathrm{A}$ & Filter \# 2, BW $=800 \mathrm{kHz}$ & $39.6 \mathrm{~dB}$ \\
\hline & $\mathrm{I}_{\mathrm{o}}=50 \mu \mathrm{A}$ & Filter \# $1, \mathrm{BW}=2.0 \mathrm{MHz}$ & $43.5 \mathrm{~dB}$ \\
\hline Noise Current & $\mathrm{I}_{\mathrm{o}}=10 \mu \mathrm{A}$ & Filter $\# 2, \mathrm{BW}=800 \mathrm{kHz}$ & $121 \mathrm{nA}_{\mathrm{rms}}$ \\
\hline \multirow{2}{*}{ Dynamic Range } & $\mathrm{I}_{\mathrm{o}}=10 \mu \mathrm{A}$ & Filter $\# 2, \mathrm{BW}=800 \mathrm{kHz}$ & $35.2 \mathrm{~dB}$ \\
\hline & $\mathrm{I}_{\mathrm{o}}=50 \mu \mathrm{A}$ & Filter \# $1, \mathrm{BW}=2.0 \mathrm{MHz}$ & $43.9 \mathrm{~dB}$ \\
\hline \multirow{2}{*}{$\begin{array}{l}\text { Power per pole } \\
\text { (simulated) }\end{array}$} & $\mathrm{I}_{\mathrm{o}}=10 \mu \mathrm{A}$ & \multirow{2}{*}{ Filter \# 1} & $191 \mu \mathrm{W}$ \\
\hline & $\mathrm{I}_{\mathrm{o}}=100 \mu \mathrm{A}$ & & $2.09 \mathrm{~mW}$ \\
\hline
\end{tabular}

Two methods were used in an attempt to alleviate the problem: first, the switching or cycling of various filter bias currents could be used to push the filter through various states, eventually leading to the desired operating point. This method does not appear to lend itself to any fixed start-up procedure and requires a great deal of patience. However, for all but the lowest levels of current biasing, circuits could be successfully started using this method. The second method, which was implemented on the most recent chip submissions, involved the inclusion of switches which could be used to temporarily force various internal nodes to predetermined voltages. This method was not found to be very effective, since the filters could not function with switches turned on, and would generally return to previous undesired states once switches were turned off. The first of the two methods was generally found to be preferable. 
A detailed investigation of the locations and origins of the multiple operating points of these biquadratic and elliptic filters was not undertaken, though it would be strongly recommended that this issue be examined if research using this CMOS circuit topology is continued in the future. A method for determining the existence of multiple operating points is described in [17], and a technique which may possibly eliminate such operating points is described in [36].

\subsection{3 - A Third Order Elliptic Low Pass Filter}

This section will describe the experimentally measured performance of a low pass elliptic log-domain filter, and will begin with the filter frequency response. The experimentally measured response of the filter is shown in Figures 4.13 and 4.14. In each plot, the bias current has been held constant at $10 \mu \mathrm{A}$ and $100 \mu \mathrm{A}$ respectively while the filter capacitance has been varied. The simulated elliptic frequency responses have been included for comparison.

The overall response of the elliptic filter was far less ideal than in either the damped integrator or the biquadratic filter cases shown previously. First, it should be mentioned that the elliptic filter was not observed to function at low values of bias current due to the existence of multiple operating points as described in the previous section. In addition, there was an unexpected peak in the response at approximately $20 \mathrm{MHz}$ which has masked the location of the zero in the elliptic transfer function for $\mathrm{I}_{\mathrm{o}}=100 \mu \mathrm{A}$ (though based on the slope of the response, the zero must have been present). The measured stopband attenuation, designed for $30 \mathrm{~dB}$, was measured to be between only 16 and $28 \mathrm{~dB}$. There is also significant peaking in the response near the cut-off frequency, though such peaking can be significantly reduced simple using simple techniques such as resistive lead compensation.

Despite these issues, the two plots demonstrate that an elliptic filter can be implemented using the circuitry and methods described in this work, and that the filter can be tuned by means of bias current or filter capacitance as expected. The implementation of an elliptic function is significant, since it verifies that both the poles and zeros can be accurately placed, a testament to the potential of this CMOS filter design approach and technique. Also note that the measured maximum cut-off frequency of the filter was $10 \mathrm{MHz}$, a figure very difficult to achieve using subthreshold MOSFET devices. 


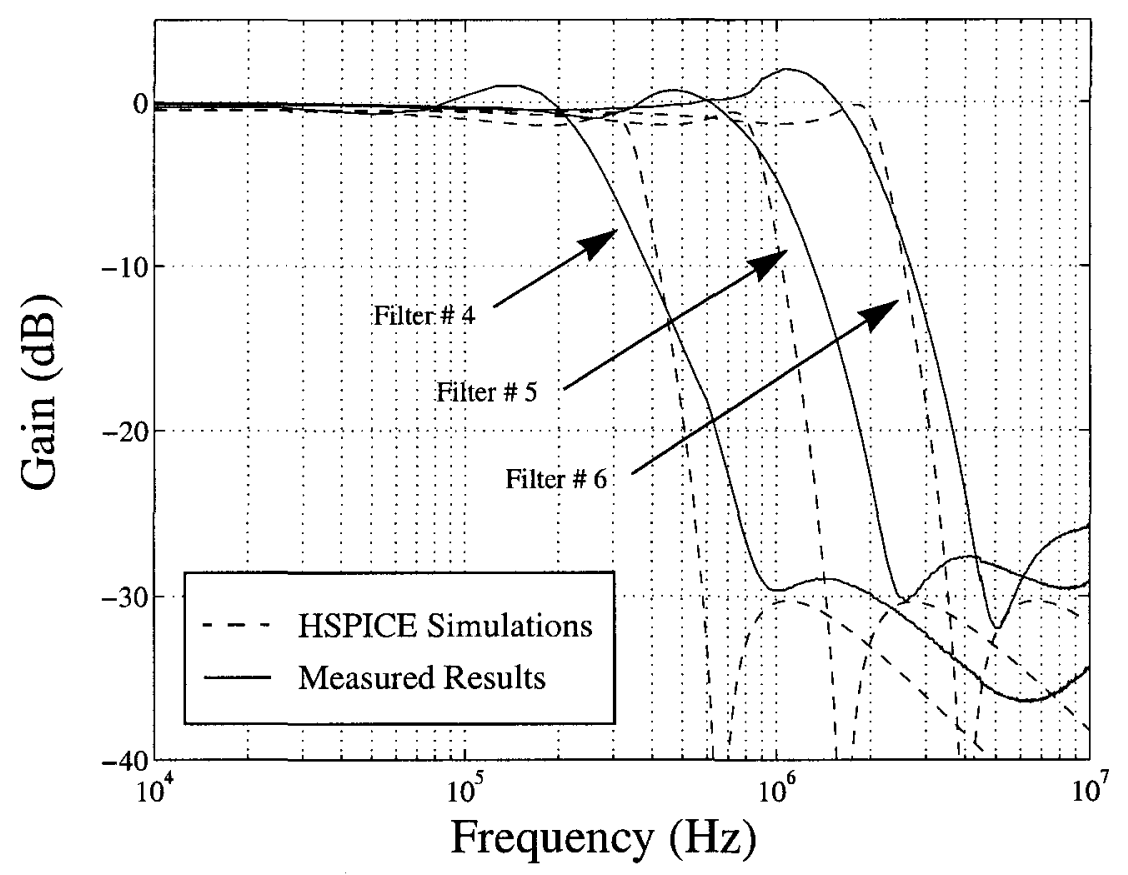

Figure 4.13 Frequency response of elliptic filter \# 4- \# 6, $I_{0}=10 \mu \mathrm{A}$

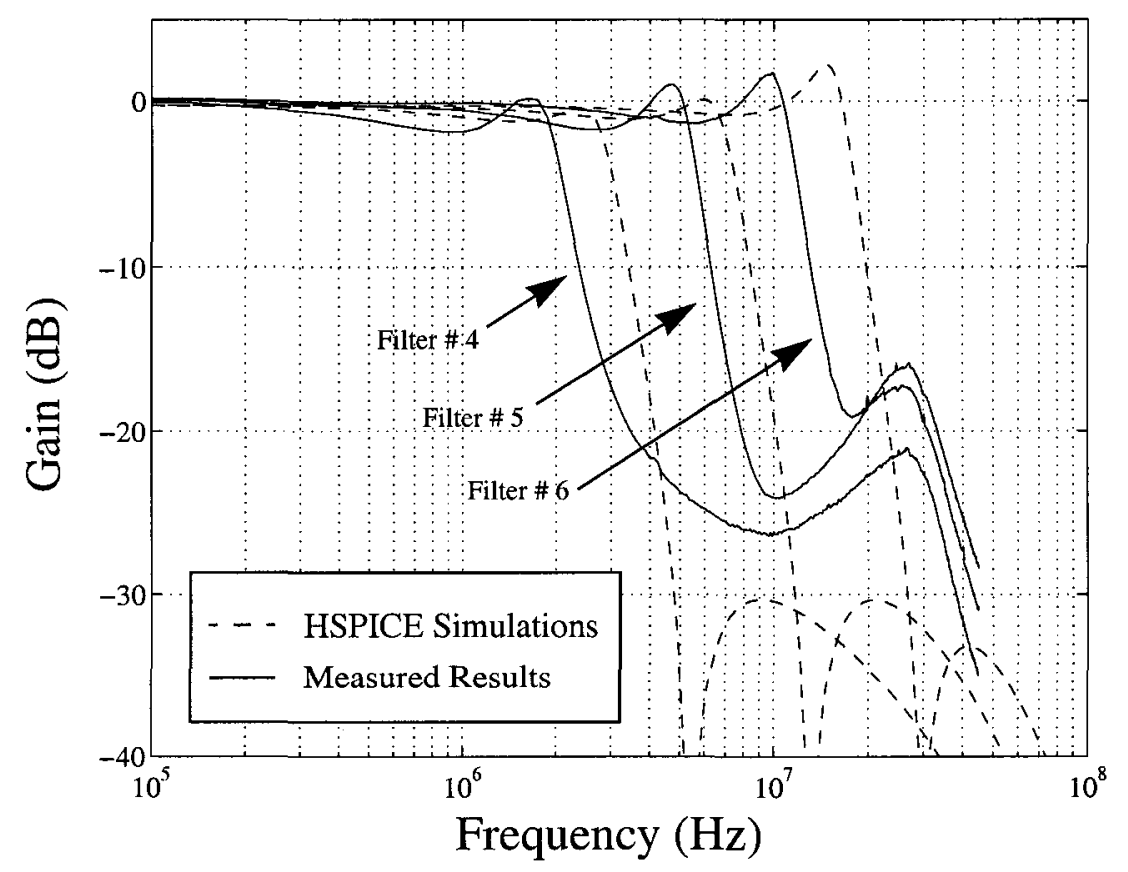

Figure 4.14 Frequency response of elliptic filter \# 4 - \# $6, I_{0}=100 \mu \mathrm{A}$ 
Next, the overall linearity of the filter was characterized using measurements of both the total harmonic distortion and the third order intercept points, and the results of each of these tests are shown in Figures 4.15 and 4.16. A bias current of $\mathrm{I}_{\mathrm{O}}=10 \mu \mathrm{A}$ and an input signal of $100 \mathrm{kHz}$ was used in each case. The THD of the filter at $\mathrm{I}_{\mathrm{in}}=0.5 \mathrm{I}_{\mathrm{o}}$ was measured to be $-39.6 \mathrm{~dB}$ and the input and output third order intercept points were measured to be $116 \mu \mathrm{A}_{\text {peak }}$ and $55 \mu \mathrm{A}_{\text {peak }}$ respectively (the difference between the input and output points reflecting the fact that the output current gain of the filter is approximately 0.5 ). Both the THD and input third order intercept points show a slight reduction from the biquadratic filter measurements, as should be expected.

The noise performance of the filter was characterized using measurements of SNR dynamic range, and output noise current. The SNR of Filter \# 6, with $\mathrm{I}_{\mathrm{o}}$ set to $10 \mu \mathrm{A}$ and a measurement bandwidth of $1.5 \mathrm{MHz}$, is shown in Figure 4.15. The maximum value of SNR was $31.2 \mathrm{~dB}$, and the dynamic range was $34.1 \mathrm{~dB}$. The output noise current over the measurement bandwidth was $134 \mathrm{nA}_{\mathrm{rms}}$. The total noise current was slightly increased with respect to the noise current for the biquadratic filter, and as a result the measured values of SNR and dynamic range show a reduction from those given in the previous section. The value of SNR has been further reduced from the biquadratic case due to the elliptic filter current gain of being 0.5 rather than unity. By increasing the bias current to $50 \mu \mathrm{A}$, the SNR and dynamic range could once again be improved by several decibels. As in previous cases, the measured noise-related performance is significantly lower than anticipated, and there may be significant room for improvement in the test and measurement set-up. A summary of the elliptic filter measurements are listed in Table 4.3.

The performance of the biquadratic and elliptic filters have been characterized, and a comparison to the performance of other log-domain filters described in the literature will now be provided. 


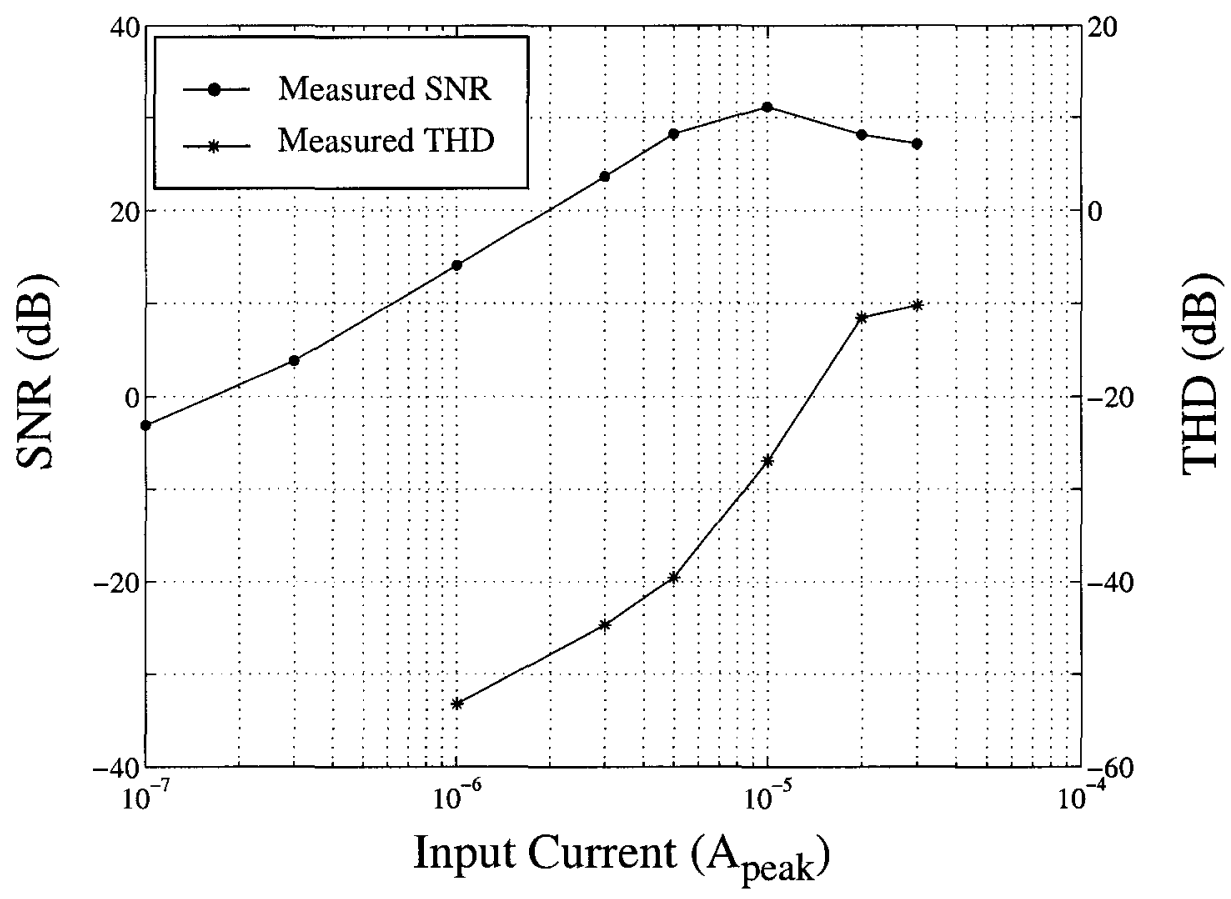

Figure 4.15: SNR and THD for filter \# $6, I_{0}=10 \mu \mathrm{A}, I_{\text {in }}=100 \mathrm{kHz}, \mathrm{BW}=1.5 \mathrm{MHz}$

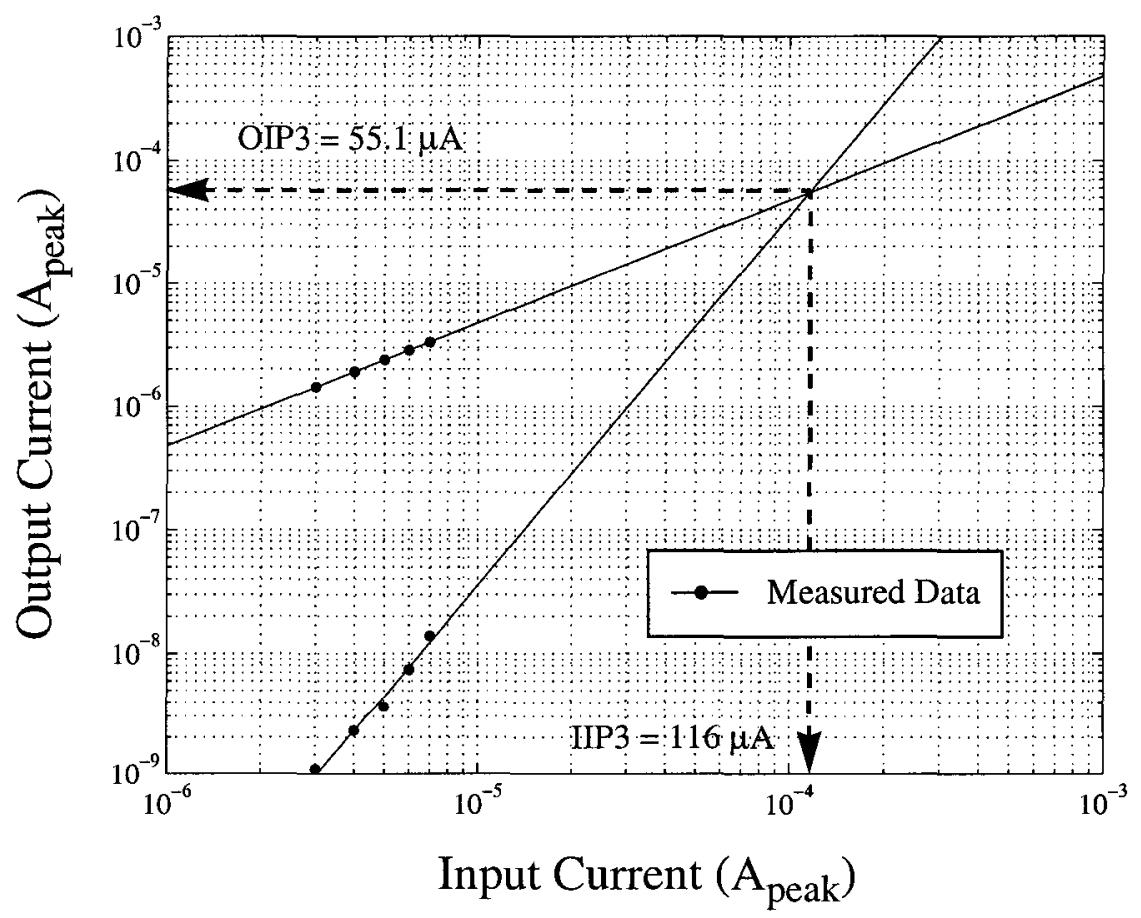

Figure 4.16 Third order intercept points for filter \# $6, I_{0}=10 \mu \mathrm{A}, I_{\text {in }}=100 \mathrm{kHz}$ 
Table 4.3 - A summary of elliptic filter performance

\begin{tabular}{|c|c|c|c|}
\hline Parameter & Bias Current & Conditions & $\begin{array}{l}\text { Experimental } \\
\text { Results }\end{array}$ \\
\hline \multirow{2}{*}{$\begin{array}{l}\text { Frequency Range } \\
\qquad\left(\mathrm{f}_{1 \mathrm{~dB}}\right)\end{array}$} & $\mathrm{I}_{\mathrm{o}}=10 \mu \mathrm{A}-100 \mu \mathrm{A}$ & Filter \#6 & $1.5 \mathrm{MHz}-10 \mathrm{MHz}$ \\
\hline & $\mathrm{I}_{\mathrm{o}}=100 \mu \mathrm{A}$ & Filter \#4 - \#6 & $1.8 \mathrm{MHz}-10 \mathrm{MHz}$ \\
\hline THD & $\mathrm{I}_{\mathrm{o}}=10 \mu \mathrm{A}$ & Filter \# 6, $\mathrm{I}_{\mathrm{in}}=0.5 \mathrm{I}_{\mathrm{o}}$ & $-39.6 \mathrm{~dB}$ \\
\hline Third Order Int. & $\mathrm{I}_{\mathrm{o}}=10 \mu \mathrm{A}$ & Filter \# 6 & $55 \mu \mathrm{A}_{\text {peak }}$ \\
\hline \multirow{2}{*}{ Maximum SNR } & $\mathrm{I}_{\mathrm{o}}=10 \mu \mathrm{A}$ & Filter \# $6, \mathrm{BW}=1.5 \mathrm{MHz}$ & $31.2 \mathrm{~dB}$ \\
\hline & $\mathrm{I}_{\mathrm{o}}=50 \mu \mathrm{A}$ & Filter \# 6, BW = 7.5 MHz & $35.7 \mathrm{~dB}$ \\
\hline Noise Current & $\mathrm{I}_{\mathrm{o}}=10 \mu \mathrm{A}$ & Filter \# $6, \mathrm{BW}=1.5 \mathrm{MHz}$ & $134 \mathrm{nA}_{\mathrm{rms}}$ \\
\hline \multirow{2}{*}{ Dynamic Range } & $\mathrm{I}_{\mathrm{o}}=10 \mu \mathrm{A}$ & \multirow{2}{*}{ Filter \# 6} & $34.1 \mathrm{~dB}$ \\
\hline & $\mathrm{I}_{\mathrm{o}}=50 \mu \mathrm{A}$ & & $38.7 \mathrm{~dB}$ \\
\hline \multirow{2}{*}{$\begin{array}{c}\text { Power per pole } \\
\text { (simulated) }\end{array}$} & $\mathrm{I}_{\mathrm{o}}=10 \mu \mathrm{A}$ & \multirow{2}{*}{ Filter \# 6} & $183 \mu \mathrm{W}$ \\
\hline & $\mathrm{I}_{\mathrm{o}}=100 \mu \mathrm{A}$ & & $2.25 \mathrm{~mW}$ \\
\hline
\end{tabular}

\section{4 - A Comparison of Filter Performance}

This section will provide a comparison of the performance of the log-domain filters designed in this work versus other log-domain filter implementations. First, a comparison will be provided between this CMOS implementation and the original bipolar implementation from which these circuits were developed. Second, a comparison will be provided between this filter implementation and all other experimentally measured CMOS implementations. It will be demonstrated that these filters compare well to other CMOS filters described in the literature.

The CMOS integrator described in this work have been mapped from an all NPN bipolar integrator described in [8]. A third order Chebychev filter was used to verify the operation of this integrator, which was implemented using Gennum GA911 technology, an inexpensive semicustom bipolar process. The measured performance specifications for this filter provide a useful benchmark for the CMOS filters described in this work. A comparison of the bipolar and CMOS filter performance is provided in Table 4.4. 
Table 4.4 - Comparison of original bipolar and CMOS filter performance

\begin{tabular}{|c|c|c|c|c|}
\hline Filter Type & $\begin{array}{c}\text { Bipolar } \\
\text { Implementation, } \\
\text { Third Order } \\
\text { Chebychev [8] }\end{array}$ & $\begin{array}{c}\text { CMOS } \\
\text { Implementation, } \\
\text { Damped } \\
\text { Integrator }\end{array}$ & $\begin{array}{c}\text { CMOS } \\
\text { Implementation, } \\
\text { Biquadratic Filter }\end{array}$ & $\begin{array}{c}\text { CMOS } \\
\text { Implementation, } \\
\text { Elliptic Filter }\end{array}$ \\
\hline Filter Order & 3 & 1 & 2 & 3 \\
\hline Ibias & $25 \mu \mathrm{A}$ & $10 \mu \mathrm{A}$ & $10 \mu \mathrm{A}$ & $10 \mu \mathrm{A}$ \\
\hline $\begin{array}{c}\text { Integrating } \\
\text { Capacitors }\end{array}$ & $\begin{array}{c}22 \mathrm{pF}, 10 \mathrm{pF}, \\
22 \mathrm{pF}\end{array}$ & $100 \mathrm{pF}$ & $100 \mathrm{pF}, 50 \mathrm{pF}$ & $\begin{array}{c}44 \mathrm{pF}, 20 \mathrm{pF}, \\
44 \mathrm{pF}, 8 \mathrm{pF}\end{array}$ \\
\hline Cut-off Frequency & $1.5 \mathrm{MHz}$ & $400 \mathrm{kHz}$ & $640 \mathrm{kHz}$ & $1.5 \mathrm{MHz}$ \\
\hline THD & - & $-44.3 \mathrm{~dB}$ & $-43.1 \mathrm{~dB}$ & $-39.6 \mathrm{~dB}$ \\
\hline 3rd Order Int. & $85 \mu \mathrm{A}_{\mathrm{peak}}$ & $203 \mu \mathrm{A}_{\mathrm{peak}}$ & $149 \mu \mathrm{A}_{\mathrm{peak}}$ & $55 \mu \mathrm{A}_{\mathrm{peak}}$ \\
\hline Maximum SNR & $42.3 \mathrm{~dB}$ & $43.4 \mathrm{~dB}$ & $39.6 \mathrm{~dB}$ & $31.2 \mathrm{~dB}$ \\
\hline Output Noise & $52.6 \mathrm{nA} \mathrm{rms}_{\text {Current }}$ & $90 \mathrm{nA} \mathrm{rms}$ & $121 \mathrm{nA}$ rms & $134 \mathrm{nA}$ rms \\
\hline Dynamic Range & $40.5 \mathrm{~dB}$ & $39.1 \mathrm{~dB}$ & $35.2 \mathrm{~dB}$ & $34.1 \mathrm{~dB}$ \\
\hline Supply Voltage & $1.2 \mathrm{~V}$ & $2.5 \mathrm{~V}$ & $2.5 \mathrm{~V}$ & $2.5 \mathrm{~V}$ \\
\hline Power / Pole & $282 \mu \mathrm{W}$ & $229 \mu \mathrm{W}$ & $191 \mu \mathrm{W}$ & $183 \mu \mathrm{W}$ \\
\hline
\end{tabular}

* $-40 \mathrm{~dB}$ HD3 Measurement

Overall, the CMOS implementation shows remarkably similar performance to the original bipolar implementation. The achieved cutoff frequencies, distortion, and power consumption per pole for the CMOS filters are on par with the original bipolar implementation. The SNR, output noise current, and dynamic range compare somewhat less favourably, directly linked to the higher noise power measured in the CMOS implementation. However, simulations indicate that noise performance observed for the CMOS filters is far from the optimal performance which can be theoretically achieved. An improved circuit board for testing, specifically one which uses lower resistances (or some other means) to implement current references, could be used to improve the noise performance. If the output noise current could by reduced by a factor of two, the experimental SNR and dynamic range of the CMOS filters would be comparable to the original bipolar implementation. 
While the relative performance of these CMOS filters is encouraging, it is important to point out that further implementations which make use of the bipolar integrator in [8] have been fabricated in more advanced bipolar processes, and operating frequencies from $30-100 \mathrm{MHz}$ have been reported [11]. Such filter cut-off frequencies are likely beyond the range which can be achieved in CMOS technology using lateral PNP devices. However, the results listed in Table 4.4 are very important, since they suggest that the performance of an inexpensive bipolar-only process can be achieved in standard submicron CMOS technology by making use of the lateral PNP devices. This is perhaps the most significant finding of this work.

It is also useful to compare the performance of the filter described in this chapter to other CMOS implementations in the literature. Although many papers have been devoted to the subject of log-domain filters in CMOS technology, very few provide experimental results. Experimentally measured filter specifications reported in the literature can be found in [13]-[19] and are summarized in Table 4.5. The results published by Python and Enz [18] and Krishnapura and Tsividis [19] deserve particular attention.

Almost all log-domain filter research in CMOS technology makes use of MOS devices operating in the subthreshold regime, such as described in the work by Python and Enz, which provides a detailed characterization of a second order filter. The authors report very low distortion, excellent noise performance, and an extremely high dynamic range of $110 \mathrm{~dB}$ (due to class $\mathrm{AB}$ operation) from their circuit. The filter was designed for a micropower application and had a cut-off frequency of $45 \mathrm{kHz}$ and a power consumption of $6 \mu \mathrm{W} /$ pole. Transistors were reported to enter into moderate inversion for currents over $2 \mu \mathrm{A}$, though the filter was shown to operate at frequencies of over $2 \mathrm{MHz}$ for a bias current of $20 \mu \mathrm{A}$. No data on distortion or noise performance was provided for this level of bias current. The reported filter specifications at a bandwidth of $45 \mathrm{kHz}$ are compared to the CMOS bipolar filter of this chapter in Table 4.5. 
Table 4.5 - Comparison of experimental CMOS filter performance reported in the literature

\begin{tabular}{|c|c|c|c|c|c|c|}
\hline Reference & $\begin{array}{c}\text { Germanovix, } \\
\text { Toumazou } \\
{[13],[14]}\end{array}$ & $\begin{array}{c}\text { Himmelbauer, } \\
\text { Andreou } \\
{[15]}\end{array}$ & $\begin{array}{l}\text { D. Masmoudi } \\
\text { et. al. [16] }\end{array}$ & $\begin{array}{c}\text { Python, Enz } \\
{[18]}\end{array}$ & $\begin{array}{l}\text { Krishnapura, } \\
\text { Tsividis [19] }\end{array}$ & This Work \\
\hline Technology & $2 \mu \mathrm{CMOS}$ & $2 \mu \mathrm{CMOS}$ & $1.6 \mu \mathrm{CMOS}$ & $\begin{array}{c}0.35 \mu \\
\text { CMOS }\end{array}$ & $\begin{array}{c}0.25 \mu \\
\text { CMOS }\end{array}$ & $\begin{array}{c}0.35 \mu \\
\text { CMOS }\end{array}$ \\
\hline Filter Order & - & 1,2 & 2 & 2 & 2 & 3 \\
\hline Ibias & $\sim 10 \mathrm{nA}$ & $10 \mathrm{nA}$ & $200 \mathrm{nA}$ & $200 \mathrm{nA}$ & $0.5 \mu \mathrm{A}$ & $10 \mu \mathrm{A}$ \\
\hline $\begin{array}{l}\text { Integrating } \\
\text { Capacitors }\end{array}$ & - & $3.3 \mathrm{pF}$ & $20 \mathrm{pF}, 200 \mathrm{pF}$ & $30 \mathrm{pF}, 30 \mathrm{pF}$ & $\begin{array}{l}180 \mathrm{pF} \\
180 \mathrm{pF}\end{array}$ & $\begin{array}{r}44 \mathrm{pF}, 20 \mathrm{pF} \\
44 \mathrm{pF}, 8 \mathrm{pF}\end{array}$ \\
\hline Cut-off Freq. & $\sim 20 \mathrm{kHz}$ & $15 \mathrm{kHz}$ & $13 \mathrm{kHz}$ & $45 \mathrm{kHz}$ & $22 \mathrm{kHz}$ & $1.5 \mathrm{MHz}$ \\
\hline $\begin{array}{c}\text { THD } \\
\left(\mathrm{I}_{\mathrm{in}}=0.5 \mathrm{I}_{\mathrm{o}}\right)\end{array}$ & $<-40 \mathrm{~dB}$ & & $<-34 \mathrm{~dB}$ & $<-55 \mathrm{~dB}$ & $-45 \mathrm{~dB}$ & $-39.6 \mathrm{~dB}$ \\
\hline Max. SNR & & & & $62 \mathrm{~dB}$ & & $31.2 \mathrm{~dB}$ \\
\hline Max. SNDR & & & & & $44.9 \mathrm{~dB}$ & $27.9 \mathrm{~dB}$ \\
\hline $\begin{array}{l}\text { Equivalent. } \\
\text { Input Noise }\end{array}$ & & & & $0.20 \mathrm{nA}_{\mathrm{rms}}$ & & $281 \mathrm{nA}_{\mathrm{rms}}$ \\
\hline Output Noise & & & & & $0.25 \mathrm{nA}_{\mathrm{rms}}$ & $134 \mathrm{nA}_{\mathrm{rms}}$ \\
\hline Dyn. Range & & & $57 \mathrm{~dB}^{*}$ & $110 \mathrm{~dB}^{* *, t}$ & $56.1 \mathrm{~dB}^{* *}$ & $34.1 \mathrm{~dB}$ \\
\hline Voltage & & & $1.2 \mathrm{~V}$ & $1.5 \mathrm{~V}$ & $1.5 \mathrm{~V}$ & $2.5 \mathrm{~V}$ \\
\hline Power / pole & & & $2.5 \mu \mathrm{W}$ & $6 \mu \mathrm{W}$ & $2.05 \mu \mathrm{W}$ & $183 \mu \mathrm{W}$ \\
\hline
\end{tabular}

\footnotetext{
* $-34 \mathrm{~dB}(2 \%)$ HD3 Measurement

** $-40 \mathrm{~dB}(1 \%) \mathrm{HD} 3$ Measurement

${ }^{\dagger}$ Class AB Operation
}

A recent contribution by Krishnapura and Tsividis [19] describes a log-domain filter implemented with lateral PNP devices, similar to that used in this work. The filter was also designed for micropower applications and had a cut-off frequency of $22 \mathrm{kHz}$ (with a maximum cut-off frequency of $41 \mathrm{kHz}$ ). The design approach in the paper differed from the one described in this work, since the authors used an enhanced mode of lateral PNP operation, in which the voltage at the gate terminal was used to increase the effective beta of the transistor and significantly decrease base current, as described in [24] and [30]. Operating in this regime allows for excellent filter linearity and is very suitable for low 
power applications, as indicated by the specifications given in Table 4.5. The reported noise performance was also very good. However, note that this enhanced mode of lateral PNP operation is confined to relatively low values of $\mathrm{V}_{\mathrm{EB}}$, and therefore to low currents, similar to the case of CMOS-subthreshold filters.

Several important conclusions can be drawn from the comparison in Table 4.5 and from the discussion above. In the realm of linearity and noise performance, the CMOSsubthreshold approach currently holds an advantage over the CMOS-bipolar approach described in this work. However, in terms of bandwidth, the bipolar based approach described in this work holds a performance advantage. Furthermore, since filter bandwidth is proportional to $\mathrm{I}_{\mathrm{o}} / \mathrm{C}$, it is reasonable to assume that, for equal capacitance, a CMOSbipolar based filter can always be expected to be capable of operation at significantly higher frequencies, at the obvious expense of increased power consumption. Also note that since the CMOS-bipolar filter in [19] reported significantly better noise performance and somewhat less distortion than the filters described in this chapter, and therefore it is reasonable to assume that the CMOS-bipolar circuits described in this work possess the potential for improved performance, particularly in terms of noise performance.

In summary, the performance characteristics of a second and a third order CMOS log-domain filter have been described. A comparison with the results with a bipolar-only filter implementation indicate that the performance of filters designed with lateral PNP transistors in $0.35 \mu$ CMOS technology are on par with the performance of filters which have be designed in an inexpensive bipolar process. When compared with other CMOS log-domain filter implementations, the filters described here represent the highest bandwidth CMOS log-domain filters reported to date. In addition, it is reasonable to assume that there is still significant room for improvement in several areas of the filter performance. 


\section{Chapter 5 - Conclusions}

\section{1 - Summary and Discussion}

The development of log-domain filters in CMOS technology using lateral bipolar transistors has been the subject of this dissertation. Many significant findings have been made, and are discussed below.

In Chapter 2, a SPICE compatible model for a lateral PNP transistor fabricated in $0.35 \mu$ CMOS technology was presented. The behaviour of the overall transistor was modelled using two individual transistors representing the desired lateral device and a parasitic vertical device. The overall model has been intended to be sufficiently simple that it could be readily developed in any CMOS technology. The model required only fifteen parameters in total, each of which could be estimated based on physical considerations, extracted from straightforward device measurements, or adopted from standard information provided by the manufacturer. The model was optimized with respect to the experimentally measured DC voltage and current characteristics, and was demonstrated to be quite adequate for the purposes of simulating the responses of log domain circuits in subsequent chapters.

Note that at high frequencies, the measured filter bandwidths were somewhat lower than those predicted by SPICE circuit simulations. While this may be attributed in part to experimental setup which was not included in simulations, it may also indicate that the model parameters which affect frequency response of the lateral bipolar transistor, and in particular the base transit time, $\tau_{f}$, may have been underestimated. Nonetheless, it should be 
clearly stated that having the ability to work from a transistor model which accurately described the behaviour of the lateral and parasitic vertical devices, even if only at relatively low frequencies, was invaluable in the development and understanding of the behaviour of these log-domain circuits.

In Chapter 3, a log-domain integrator was designed and characterized. The integrator which made use of both lateral PNP transistors and MOSFET transistors operating in strong inversion was developed from an existing bipolar design. Many of the considerations involved in the mapping from bipolar to CMOS technology were described, and the design of the supporting circuitry was also discussed. Experimental results indicate that the integrator was capable of operation at $10 \mathrm{MHz}$ and above, that the total harmonic distortion at a modulation index of $50 \%$ was better than $-40 \mathrm{~dB}$ for moderate levels of bias current, and that the maximum SNR and dynamic range of the filter were on the order of $40 \mathrm{~dB}$ each. The integrator could operate from a $2.5 \mathrm{~V}$ supply, and consumed $229 \mu \mathrm{W}$ of current when a bias current of $10 \mu \mathrm{A}$ was used.

A direct comparison of simulated and experimental values was made for all measurements of the integrator. The agreement was reasonable for all measurements except for those related to the noise performance. The noise floor, calculated over a bandwidth of $640 \mathrm{kHz}$, was simulated and experimentally measured to be $10.3 \mathrm{nA}_{\mathrm{rms}}$ and $90 \mathrm{nA}_{\mathrm{rms}}$ respectively, indicating that the experimental setup was likely far from optimal. The suggests that significantly higher SNR and dynamic range can still be achieved through improvements to this experimental setup.

In Chapter 4, a biquadratic low pass filter and a third-order elliptic low pass filter were each designed and characterized. Methods of signal flow graphs and the operational simulation of LC ladders were used to design these filters, and it was verified experimentally that such methods could indeed be used to accurately synthesize the desired filter responses, including the positioning of both poles and zeros, a significant result. Experimental results indicate that the elliptic filter was capable of operation at $10 \mathrm{MHz}$, that the total harmonic distortion at a modulation index of $50 \%$ was just under $-39.6 \mathrm{~dB}$ for moderate levels of bias current, and that the maximum SNR and dynamic range of the filter were $31.2 \mathrm{~dB}$ and 34.1 
$\mathrm{dB}$ respectively. The filter could also operate from a $2.5 \mathrm{~V}$ supply and consumed $183 \mu \mathrm{W} /$ pole when a bias current of $10 \mu \mathrm{A}$ was used.

As was previously mentioned in Chapter 4, one of the significant contributions of this work is the demonstration that log domain filters in CMOS technology can be designed to operate at cut-off frequencies of up to $10 \mathrm{MHz}$ and above, a figure very difficult to achieve using weakly-inverted devices. When compared with other CMOS implementation in the literature, these filters represent the highest bandwidth CMOS log-domain filters reported to date. The capacitances used in this work were in many cases placed off-chip, and in general were not the minimum size capacitors which could have been used. It is reasonable to assume that these filters could operate at higher frequencies than reported here. In addition, as in the case of the integrator, the discrepancy in simulated and measured noise performance suggest that significantly higher SNR and dynamic range could be achieved through improved experimental setup.

Also as mentioned in Chapter 4, another significant contribution of this work is the demonstration that circuit performance similar to that of a semicustom bipolar-only process could be achieved in standard submicron CMOS technology though the use of lateral PNP devices. The use of these devices is not often described in the literature, and semiconductor manufacturers generally do not provide models for such devices, yet their performance has been shown to be quite adequate for this mid-frequency application. In addition, since the overall performance of lateral bipolar transistors can be expected to improve as the dimensions of CMOS technology are reduced, such devices hold significant potential and may find a variety of uses and applications in the future.

The characteristics of a first, second, and third order log-domain filter have been thoroughly investigated. The comparison of the relative performance of the three is of great value, since it provides insight into the performance (or degradation in performance) which can be expected as further high-order filters are implemented. Overall, the measured performance is very encouraging, and it has clearly been indicated that log-domain filters can be successfully implemented in standard CMOS technology using the methods described in this work. 


\section{2 - Directions for future work}

Since the log-domain filtering still represents a new frontier in the realm of filter design, there are several directions which this research might take in the future. To begin, it should be reiterated that measured performance reported in this work may not represent the limit of performance which can be achieved with these circuits or in this technology, and that further investigations of these limits would be of great interest. However, in more general terms, several potential directions of future research are described below.

First, since the overall performance of lateral PNP transistors can be expected to generally improve as the dimensions of CMOS technology are reduced, the implementation of such filters in $0.18 \mu$ or $0.13 \mu$ CMOS technologies would be a logical progression of this work. Note however that the improvement in performance in any particular technology cannot be taken for granted, since a vast number of variables, such as the doping levels within the process, can affect the lateral bipolar performance. Implementation of these filters in a triple-well CMOS process would also open up many new possibilities, since NPN transistors as well as PNP transistors could then be implemented. In addition, a high frequency characterization of these devices, in $0.35 \mu$ CMOS or any other submicron CMOS process, would provide both useful information for device modelling and further insight into the potential for these filters.

Secondly, an investigation of other integrator circuit topologies would be of great value. As a straightforward extension, the integrator and filters described here could be implemented using differential or class- $A B$ structures, which could lead to significant improvements in the THD and dynamic range which could be attained with these filters. Also note that there are other integrator structures in the literature which could also be implemented in standard CMOS technology, including those in [10] and [19]. A detailed investigation of such structures could ultimately lead to the implementation of entirely new circuit topologies which best take advantage of the capabilities of CMOS processes.

Finally, if the ultimate goal in implementing log-domain filters in CMOS technology, rather than bipolar technology, is to make this filter design technique more 
applicable to industry, then a thorough investigation of many practical implementation issues must be addressed. It would be of great value to examine the effects of circuit nonidealities on performance of these CMOS log-domain filters, in a similar manner to that described in [32]. It would also be crucial to obtain a full understanding of the multiple operating points which have been observed to arise in these filters, as described in [17]. Though undertaking such topics is perhaps less glamourous than many other aspects of log-domain filter design, the contribution of such investigations would be significant.

In conclusion, this dissertation has established that log-domain filters can be successfully implemented in standard CMOS technology using the lateral bipolar transistors. The CMOS log-domain filters developed in this work have the highest bandwidth of any CMOS log-domain filters reported to date. The potential for the lateral bipolar device, fabricated in standard submicron CMOS technology has also clearly been demonstrated.

Plaudite, amici, res finita est. 


\section{Appendix}

A table of values which have been used in the calculation of the theoretical first-order lateral PNP model in Chapter 2 are provided below.

Table A.1: Parameter values for the lateral PNP transistor in $0.35 \mu$ CMOS technology

\begin{tabular}{|c|c|c|c|}
\hline \multicolumn{2}{|c|}{ Parameter Descrption } & \multirow{2}{*}{$\frac{\text { Variable }}{N_{E}}$} & \multirow{2}{*}{$\frac{\text { Value }}{1 \times 10^{20} \mathrm{~cm}^{-3}}$} \\
\hline Emitter Doping & & & \\
\hline Emitter Radius & & $\mathrm{R}_{\mathrm{E}}$ & $0.65 \mu \mathrm{m}$ \\
\hline Emiiter Depth & & $\mathrm{D}_{\mathrm{E}}$ & $0.15 \mu \mathrm{m}$ \\
\hline \multirow[t]{2}{*}{ Effective Emitter Width } & lateral & $\mathrm{W}_{\mathrm{E}}$ & $0.65 \mu \mathrm{m}$ \\
\hline & vertical & $\mathrm{W}_{\mathrm{E}}$ & $0.25 \mu \mathrm{m}$ \\
\hline \multirow[t]{3}{*}{ Emitter Base Junction Area } & lateral & $A_{E B}$ & $0.87 \mu \mathrm{m}^{2}$ \\
\hline & vertical & $\mathrm{A}_{\mathrm{EB}}$ & $0.87 \mu \mathrm{m}^{2}$ \\
\hline & total & $A_{E B}$ & $1.74 \mu \mathrm{m}^{2}$ \\
\hline Emitter Intrinic Concentration & & $\mathrm{n}_{\mathrm{ie}}$ & $1.68 \times 10^{11} \mathrm{~cm}^{-3}$ \\
\hline Emitter Minority Diffusion Coefficient & & $D_{n}$ & $6.0 \mathrm{~cm}^{2} / \mathrm{s}$ \\
\hline \multirow[t]{2}{*}{ Base Doping } & bulk n-well & $\mathrm{N}_{\mathrm{B}}$ & $4.0 \times 10^{16} \mathrm{~cm}^{-3}$ \\
\hline & channel region & $\mathrm{N}_{\mathrm{B}}$ & $8.5 \times 10^{16} \mathrm{~cm}^{-3}$ \\
\hline \multirow[t]{2}{*}{ Effective Base Width } & lateral & $\mathrm{W}_{\mathrm{B}}$ & $0.30 \mu \mathrm{m}$ \\
\hline & vertical & $\mathrm{W}_{\mathrm{B}}$ & $1.3 \mu \mathrm{m}$ \\
\hline Base Intrinsic Concentration & & $\mathrm{n}_{\mathrm{i}}$ & $1.5 \times 10^{10} \mathrm{~cm}^{-3}$ \\
\hline Base Minority Diffusion Coefficient & & $\mathrm{D}_{\mathrm{p}}$ & $12.5 \mathrm{~cm}^{2} / \mathrm{s}$ \\
\hline Collector Doping & lateral & $\mathrm{N}_{\mathrm{C}}$ & $1 \times 10^{20} \mathrm{~cm}^{-3}$ \\
\hline \multirow[t]{2}{*}{ Base Collector Junction Area } & lateral & $\mathrm{A}_{\mathrm{CB}}$ & $20 \mu \mathrm{m}^{2}$ \\
\hline & vertical & $\mathrm{A}_{\mathrm{CB}}$ & $92 \mu \mathrm{m}^{2}$ \\
\hline \multirow[t]{2}{*}{ Base Collector Junction Capacitance } & lateral & $\mathrm{C}_{\mathrm{BC}}$ & $10 \mathrm{fF}$ \\
\hline & vertical & $\mathrm{C}_{\mathrm{BC}}$ & $25 \mathrm{fF}$ \\
\hline
\end{tabular}




\section{References}

[1]

G.W. Roberts and V. W. Leung, "Design and Analysis of Integrator-Based Log-Domain Filter Circuits", Kluwer Academic Publishers, Boston, Massachusetts, 2000.

[2] R. W. Adams, "Filtering in the Log Domain", Preprint \# 1470, presented at the 63rd AES Conference, New York, New York, May 1979.

[3] D. Frey, "Log-Domain Filtering: An Approach to Current Mode Filtering", IEE Proceedings, Vol 140, No. 6, pp. 406-416, Dec. 1993.

[4] D. Perry and G. W. Roberts, "Log-Domain Filters based on LC Ladder Synthesis", Proc. IEEE International Symposium on Circuits and Systems, Vol. 1, pp311-314, May 1995.

D. Perry and G. W. Roberts, "Design of Log-Domain Filters Based of the Operational Simulation of LC-Ladders", IEEE Transactions on Circuits and Systems II, Vol. 43, No. 11, pp. 763-773, Nov. 1996.

[6] A. S. Sedra and P. Brackett, "Filter Theory and Design: Active and Passive", Matrix Publishers, Portland, Oregon, 1978.

E. Seevinck, "Companding Current-Mode Integrator: A New Circuit Principle for Continuous-Time Monolithic Filters", Electronics Letters, Vol. 26, No. 24, pp. 2046-2047, Nov. 1990.

M. N. El-Gamal and G. W. Roberts, "A 1.2 V NPN-Only Log-Domain Integrator", IEEE International Symposium on Circuits and Systems, Vol. II, pp. 681-684, May 1999.

D. Frey, "Log-Domain Filtering for RF Applications", IEEE Journal of Solid State Circuits, Vol. 31, No. 10, pp. 1468-1475, Oct. 1996. Log-Domain Filter", IEEE Journal of Solid State Circuits, Vol. 32, pp. 1968-1978, Dec. 1997. 
M. N. El-Gamal, R. A. Baki, and A. Bar-Dor, "A 30-100 MHz NPN-Only Variable-Gain Calss-AB Instantaneous Companding Filters for $1.2 \mathrm{~V}$ Applications", IEEE Journal of Solid State Cirucits, Vol. 35, No. 12, Dec. 2000.

C. Toumazou, J. Ngarmnil, and T. S. Lande, "Micropower Log Domain Filter for Electronic Cochlea", Electronics Letters, Vol. 30, pp. 1839-1841, Oct. 1994.

W. Germanovix and C. Toumazou, "Design of a Micropower CurrentMode Log-Domain Analog Cochlear Implant", IEEE Transactions on Circuits and Systems II, Vol. 4, No. 10, pp. 1023-1046, Oct. 2000.

W. Himmelbauer and A. G. Andreou, "Log-Domain Circuits in Subthreshold MOS", Proceedings of the 40th Midwest Symposium on Circuits and Systems, Vol. 1, pp. 26-30, Aug. 1998.

D. Masmoudi et. al., "A New Current Mode Synthesis Method for Dynamic Translinear Filters and Its Application in Hearing Aids", IEEE International Conference on Electronics, Circuits and Systems, Vol. 2, pp. 337-340, Sept. 1998.

R. M. Fox and M. Nagarajan, "Multiple Operating Points in a CMOS LogDomain Filter", IEEE Transactions on Circuits and Systems II, Vol 4, No. 6, pp. 705-710, June 1999.

[18] D. Python and C. C. Enz, "A Micropower Class-AB CMOS Log-Domain Filter for DECT Applications", IEEE Journal of Solid-State Circuits, Vol. 36, No. 7, pp. 1067-1075, July 2001.

N. Krishnapura and Y. Tsividis, "A Micropower Log-Domain Filter Using Enhanced Lateral PNPs in a $0.25 \mu$ CMOS process", IEEE International Symposium on VLSI Circuits, pp. 179-182, June 2001.

D. Frey, "C-Log Domain Filters", IEEE International Symposium on Cirucits and Systems, Vol. 1, pp. 176-179, May 2000.

G. J. Yu et. al., "Design of Log Domain Low-Pass Filters by MOSFET Square Law”, IEEE Asia Pacific Conference, pp. 9-12, Aug. 2000. 
I. E. Getreu, "Modelling the Bipolar Transistor", Tektronix Inc., Beaverton, Oregon, 1978.

V. Van, M. J. Deen, J. Kendall, D. S. Malhi, S. Voingescu and M. Schroter, "D.C. Extraction of Base and Emitter Resistances in Polysilicon Emitter npn BJTs", Canadian Journal of Physics, Vol. 74, pp. S172-S176, 1996. 
[34] D. K. Schroder, "Semiconductor Material and Device Characterization", John Wiley and Sons, New York, NY, 1990.

[35] M. Van Valkenburg, "Analog Filter Design", Holt, Rinehart and Winston, New York, New York, 1982.

[36] J. Georgiou and C. Toumazou, "An Operating Point Elimination Technique for Weak Inversion Log-Domain Filters with Multiple Operating Points", IEEE International Symposium on Circuits and Systems, Vol 1, pp. 153155, May 2001. 